\title{
MESURES DE DÉFAUT DE COMPACITÉ, APPLICATION AU SYSTÈME DE LAMÉ
}

\author{
Par Nicolas BURQ et GiLles LEBEAU
}

RÉSUMÉ. - On définit des mesures de défaut de compacité pour les systèmes aux limites vérifiant la condition de Lopatinski uniforme au bord et on utilise ces notions pour étudier la propagation de l'énergie pour les solutions du système de Lamé.

(c) 2001 Éditions scientifiques et médicales Elsevier SAS

ABSTRACT. - We define the microlocal defect measures for boundary value systems satisfying the strong Lopatinski condition and we apply these notions to the study of the asymptotic propagation of the energy for the solutions of the Lamé system.

(C) 2001 Éditions scientifiques et médicales Elsevier SAS

\section{Introduction}

On se propose dans cet article d'étudier les solutions d'un système d'équations aux dérivées partielles, d'ordre 2, à symbole principal scalaire et réel, satisfaisant des conditions aux limites vérifiant la condition de Lopatinski uniforme. L'étude de la propagation de la polarisation pour de tels systèmes a été faite par N. Denker [3] à l'intérieur et par C. Gérard [4] près des rayons strictement glissants ou strictement diffractifs, dans un cadre $C^{\infty}$. Notre étude se limite au cadre $H^{1}$ mais permet de généraliser les résultats connus antérieurement (nous supprimons les hypothèses sur la nature micro-locale des points près desquels l'étude est faite) et d'en donner une version quantitative. Plus précisément, pour toute suite de solutions d'un tel système, de norme $H^{1}$ bornée nous définirons une mesure de défaut de compacité micro-locale donnant une description quantitative de la polarisation asymptotique de la suite. Cette définition généralise au cas des systèmes aux limites la notion introduite par P. Gérard [5] et L. Tartar [15]. On démontrera ensuite un théorème de propagation permettant de calculer la mesure (et donc la polarisation) le long d'un flot que nous définirons. Dans le cas d'une unique équation des ondes, ce programme a déjà été réalisé par P. Gérard [5] et L. Tartar [15] à l'intérieur, P. Gérard, E. Leichtnam [6], G. Lebeau [10] pour la condition aux limites de Dirichlet et par H. Koch-D. Tataru [9] pour une condition aux limites absorbante, dans un cadre différent (on pourra aussi consulter l'étude de L. Miller [12]). On donnera également une application de notre résultat à l'étude du système de Lamé. Dans le cas où l'énergie de l'onde longitudinale tend vers 0 , on établira l'équation de propagation exacte pour la polarisation de l'onde transversale. On montrera que l'onde transversale est polarisée perpendiculairement à la direction de propagation, qu'aux points de réflexion transverses situés dans la région hyperbolique pour l'onde longitudinale (points de réflexion et transmission partielles), d'une part la polarisation est aussi orthogonale à la normale au bord et d'autre part la polarisation sortante est égale à la polarisation rentrante. 
Près des points hyperboliques pour l'onde transversale mais elliptiques pour l'onde longitudinale (points de réflexion totale), on donnera l'expression explicite de la polarisation sortante en fonction de la polarisation rentrante et enfin, près des points glissants, on établira l'équation de propagation donnant la polarisation à tout instant, à partir de la polarisation initiale. Cette description complète nous permettra de démontrer une conjecture de G. Lebeau et E. Zuazua sur le taux de décroissance pour le système 3-dimensionnel de la thermoélasticité, donnant une condition nécessaire et suffisante pour la décroissance exponentielle de l'énergie des solutions de ce système.

Le papier est organisé comme suit : Au §2, nous construisons d'abord au §2.1 (proposition 2.5) des mesures d'énergie microlocales $\mu$ pour les suites bornées de $H^{1}$ de solutions du système

$$
\left(\partial_{x}^{2}+R+M_{0} \partial_{x}+M_{1}\right) u_{k}=v_{k}
$$

dans le demi-espace $x>0$, sans conditions aux limites. Ici, la suite $\left(v_{k}\right)$ converge fortement vers 0 dans un espace convenable, l'opérateur $R$ est scalaire de degré 2 et les $M_{j}$ sont des opérateurs matriciels de degré $j$. Nous avons choisi le cadre pseudo-différentiel tangentiel pour les opérateurs $R, M_{0}$ et $M_{1}$.

$\mathrm{Au} \S 2.2$, nous calculons l'équation de propagation vérifiée par la mesure $\mu$ lorsque la suite $\left(u_{k}\right)$ vérifie une condition aux limites de type Lopatinski (lemmes 2.9 et 2.10).

Le $\$ 3$ est consacré au théorème de propagation dont la démonstration est purement géométrique et n'utilise (sous la condition que $\mu$ ne charge pas les points strictement diffractifs) que l'équation vérifiée par $\mu$ dans l'espace cotangent (théorème 1). Nous vérifions également que cette dernière condition est automatiquement satisfaite lorsque $\mu$ provient d'une suite $\left(u_{k}\right)$ de solutions de (1.1) vérifiant une condition aux limites de type Lopatinski (\$3.4).

Dans le $\S 4$, nous appliquons les constructions précédentes au système de Lamé. Le $\$ 5$ est consacré à la description du théorème de propagation pour l'onde transversale lorsque l'énergie de l'onde longitudinale est négligeable. Enfin, le $\$ 6$ démontre (et précise) une conjecture de G. Lebeau et E. Zuazua [11] sur la décroissance exponentielle de l'énergie pour les solutions du système de la thermoélasticité en dimension 3 (théorèmes 3 et 4).

Nous avons rassemblé dans une annexe quelques résultats de la théorie des problèmes aux limites dans le cas des points microlocalement hyperboliques ou elliptiques, présentés dans le cadre des mesures de défaut de compacité. Pour une exposition plus générale et plus complète, nous invitons le lecteur à se référer sur ce sujet aux livres de Chazarain et Piriou [2], Chapitre V pour la théorie elliptique et L. Hörmander [8], chapitre 24.2 pour la théorie hyperbolique.

\section{Mesures : notations et conventions}

Soit $X$ un espace métrique localement compact, dénombrable à l'infini. On munit $\mathbb{C}^{N}$ de sa structure hermitienne standard

$$
\left(z \mid z^{\prime}\right)=\sum_{j=1}^{N} z_{j} \bar{z}_{j}^{\prime} ; \quad|z|=(z \mid z)^{1 / 2} .
$$

Soit $C_{0}^{0}\left(X, \operatorname{End}\left(\mathbb{C}^{N}\right)\right)$ l'espace des fonctions $x \mapsto A(x)$ continues sur $X$, à support compact, à valeur dans les matrices complexes $N \times N$. On munit cet espace de la norme

$$
\|A\|=\sup _{x \in X}\left\{\sup _{z \in \mathbb{C}^{N},|z| \leqslant 1}|A(x)(z)|\right\} .
$$


On appelle mesure (vectorielle) sur $X$ un élément du dual $\mathcal{M}_{X}$ de l'espace $C_{0}^{0}\left(X, \operatorname{End}\left(\mathbb{C}^{N}\right)\right)$. Une mesure (vectorielle) $\mu$ sur $X$ est donc la donnée d'une matrice $N \times N\left(\mu_{j, i}\right)_{1 \leqslant i, j \leqslant N}$ de mesures boréliennes sur $X$ avec

$$
\langle\mu, A\rangle=\sum_{i, j} \int_{X} a_{i, j}(x) \mathrm{d} \mu_{j, i} .
$$

La mesure $\mu$ est hermitienne lorsqu'elle vérifie

$$
A^{*}=A \Rightarrow\langle\mu, A\rangle \in \mathbb{R}
$$

ce qui équivaut à $\mu_{i, j}=\overline{\mu_{j, i}}$ pour tout $i, j$.

La mesure $\mu$ est hermitienne positive lorsqu'elle vérifie

$$
A^{*}=A \geqslant 0 \Rightarrow\langle\mu, A\rangle \geqslant 0 .
$$

Pour $\mu \in \mathcal{M}_{X}$, la mesure conjuguée $\bar{\mu}$ est définie par

$$
\langle\bar{\mu}, A\rangle=\overline{\left\langle\mu, A^{*}\right\rangle}
$$

On a $\overline{(\bar{\mu})}=\mu$ et, pour $\lambda \in \mathbb{C}, \overline{(\lambda \mu)}=\bar{\lambda} \bar{\mu}$. La mesure $\mu$ est hermitienne si et seulement si on a $\bar{\mu}=\mu$. Toute mesure $\mu$ se décompose en somme $\mu=\operatorname{Re} \mu+i \operatorname{Im} \mu$, où $\operatorname{Re} \mu=\frac{\mu+\bar{\mu}}{2}$ et $\operatorname{Im} \mu=\frac{\mu-\bar{\mu}}{2 i}$ sont hermitiennes. Une mesure positive $\mu$ est hermitienne. En effet on a

$$
\langle\operatorname{Im} \mu, A\rangle=0 \quad \text { pour tout } A \text { vérifiant } \mathrm{A}^{*}=A \geqslant 0 ;
$$

en choisissant $A=\left(a_{i, j}\right)$ avec $a_{i j} \equiv 0$ sauf lorsqu'ils prennent deux valeurs données, on en déduit $\operatorname{Im} \mu=0$ donc $\bar{\mu}=\mu$.

Si $B$ est un borélien de $X$ et $\mu \in \mathcal{M}_{X}$ la mesure $\mathbf{1}_{B} \mu \in \mathcal{M}_{X}$ est définie par

$$
\left\langle\mathbf{1}_{B} \mu, A\right\rangle=\sum_{i, j} \int_{B} a_{i j}(x) \mathrm{d} \mu_{j, i} .
$$

On dit que la mesure $\mu$ ne change pas $B$ si $\mathbf{1}_{B} \mu=0$ et que $\mu$ est portée par $B$ si $\mathbf{1}_{B} \mu=\mu$. Si $\mu$ est hermitienne (positive), $\mathbf{1}_{B} \mu$ l'est également (car on peut supposer $B$ borné; on a alors $\mathbf{1}_{B} \in L^{1}(|\mu|)$, où $|\mu|=\sum_{i, j}\left|\mu_{j, i}\right|$, donc il existe une suite $\left(\varphi_{n}\right) \in C_{0}^{0}(X)$ de fonctions positives vérifiant $\lim \varphi_{n}=\mathbf{1}_{B}$ dans $L^{1}\left(\left|\mu_{j, i}\right|\right)$ pour tout $i, j$, donc $\left\langle\mathbf{1}_{B} \mu, A\right\rangle=\lim _{n}\left\langle\mu, \varphi_{n} A\right\rangle$ ).

Nous adopterons les conventions suivantes.

1. Si $F$ est fermé dans $X,\left.\mu\right|_{F}$ est la mesure $l_{F} \mu$ c'est-à-dire

$$
\left.\mu\right|_{F} \in \mathcal{M}_{X}:\left\langle\left.\mu\right|_{F}, A\right\rangle=\left\langle\mathbf{1}_{F} \mu, A\right\rangle, \quad A \in C_{0}^{0}\left(X, \operatorname{End}\left(\mathbb{C}^{N}\right)\right) .
$$

2. Si $U$ est ouvert dans $X,\left.\mu\right|_{U}$ est la mesure restriction de $\mu$ à $U$; c'est-à-dire

$$
\left.\mu\right|_{U} \in \mathcal{M}_{U}:\left\langle\left.\mu\right|_{U}, A\right\rangle=\langle\mu, A\rangle, \quad A \in C_{0}^{0}\left(U, \operatorname{End}\left(\mathbb{C}^{N}\right)\right) .
$$

En particulier, si $F$ est fermé, $\mu$ ne charge pas $F$ si et seulement si $\left.\mu\right|_{F}=0$, et $\mu$ est portée par $F$ si et seulement si $\left.\mu\right|_{(X \backslash F)}=0$. 


\subsection{Mesures au bord, notations}

Soit $Y=\left\{y \in \mathbb{R}^{d},|y|<1\right\}$ la boule unité de $\mathbb{R}^{d}$ et $X$ l'espace produit

$$
X=] 0,1[\times Y .
$$

On note $(x, y), x \in] 0,1[, y \in Y$, le point courant de $X$. On considère $X$ comme variété à bord en posant

$$
\bar{X}=[0,1[\times Y, \quad \partial X=\{x=0\} \times Y .
$$

Soit $N \geqslant 1$ un entier. On notera

$$
L^{2}=L^{2}\left(X ; \mathbb{C}^{N}\right), \quad H^{1}=H^{1}\left(X, \mathbb{C}^{N}\right)
$$

les espaces de Hilbert de fonctions vectorielles $u=\left(u_{1}, \ldots, u_{N}\right)$ définies sur $X$ à valeurs dans $\mathbb{C}^{N}$, munis de leur norme

$$
\begin{aligned}
& |u|_{L^{2}}^{2}=\int_{X} \sum_{j=1}^{N}\left|u_{j}\right|^{2} \mathrm{~d} x \mathrm{~d} y, \\
& |u|_{H^{1}}^{2}=|u|_{L^{2}}^{2}+\int_{X} \sum_{j=1}^{N}\left|\nabla u_{j}\right|^{2} \mathrm{~d} x \mathrm{~d} y,
\end{aligned}
$$

où

$$
\nabla f=\left(\frac{\partial f}{\partial x}, \frac{\partial f}{\partial y_{1}}, \ldots, \frac{\partial f}{\partial y_{d}}\right)
$$

Soit $R=R\left(x, y, D_{y}\right)$ un opérateur pseudo-différentiel scalaire $\left(C^{\infty}\right)$ tangentiel classique de degré 2 (voir [8, Chap XVIII]), auto-adjoint, défini au voisinage de $[0,1] \times \bar{Y}$, de symbole principal réel $r(x, y, \eta)$; on définit les fonctions $r_{0}$ et $r_{1}$ par

$$
r(x, y, \eta)=r_{0}(y, \eta)+x r_{1}(y, \eta)+\mathrm{O}\left(x^{2}\right) .
$$

On supposera que la fonction homogène de degré 2 en $\eta, r(x, y, \eta)$ vérifie

$$
\frac{\partial r}{\partial \eta} \neq 0 \quad \text { pour }(x, y) \in \bar{X} \text { et } \eta \neq 0 \text {. }
$$

Soient également $M_{0}\left(x, y, D_{y}\right), M_{1}\left(x, y, D_{y}\right)$ des matrices $N \times N$ d'opérateurs pseudodifférentiels $\left(C^{\infty}\right)$ tangentiels classiques définis au voisinage de $[0,1] \times \bar{Y}$, de degrés respectifs 0 et 1 , de symboles principaux $m_{0}(x, y, \eta), m_{1}(x, y, \eta)$. On note $P$ l'opérateur de degré 2 :

$$
P=\left(\partial_{x}^{2}+R\right) \operatorname{Id}+M_{0} \partial_{x}+M_{1} .
$$

Le symbole principal $p$ de $P$ est scalaire et vaut

$$
p=-\xi^{2}+r(x, y, \eta)
$$

Soit $Y^{\prime}$ un petit voisinage ouvert de $\bar{Y}$ tel que les opérateurs $R, M_{0}$ et $M_{1}$ sont bien définis au voisinage de $[0,1] \times \overline{Y^{\prime}}$. Soit $K$ un compact de $Y^{\prime}$ dont l'intérieur contient $\bar{Y}$. 
On se donne une suite $\left(u^{k}(x, y)\right)_{k \in \mathbb{N}}$, convergeant fortement vers 0 dans l'espace $H^{2}(] 0,1\left[; H^{-s}\left(Y^{\prime}\right)\right)$ pour un $s>0$ et à support dans $[0,1] \times K$. La suite $v_{k}=P u_{k}$ est alors bien définie dans $L^{2}(] 0,1\left[; H^{-s-2}\left(Y^{\prime}\right)\right)$.

On suppose que la suite $\left(u^{k}\right)_{k \in \mathbb{N}}$ est bornée dans l'espace $H^{1}(] 0,1\left[\times Y^{\prime}\right)$ et que la restriction $v^{k}$ de $P u^{k}$ à $] 0,1[\times Y$ vérifie

$$
\left.P u^{k}\right|_{] 0,1[\times Y}=v^{k} \in G=\left\{v \in L^{2} \text { et } \partial_{x} v \in L^{2}(] 0,1\left[, H^{-1}(Y)\right)\right\},
$$

et la suite $\left(v^{k}\right)$ converge fortement vers zéro dans $G$.

Remarque 2.1.-

- La suite $\left(u^{k}\right)$ converge faiblement vers 0 dans $H^{1}(] 0,1\left[\times Y^{\prime}\right)$.

- Si $\varphi \in C_{0}^{\infty}\left(Y^{\prime}\right)$ est égale à 1 près de $\bar{Y}$, alors la suite $\left(\varphi u^{k}\right)$ vérifie les mêmes hypothèses que la suite $\left(u^{k}\right)$.

- Supposons que les opérateurs $R, M_{0}$ et $M_{1}$ sont différentiels et soit $\left(f^{k}\right)$ une suite de limite faible nulle dans $H^{1}(] 0,1\left[\times Y^{\prime}\right)$, solution de $P f^{k}=0$. Pour $\varphi \in C_{0}^{\infty}\left(Y^{\prime}\right)$ égale à 1 près de $\bar{Y}$, la suite $\left(\varphi f^{k}\right)$ vérifie les hypothèses précédentes avec $v^{k}=0$ (pour un certain choix de $K$ ).

LEMME 2.2.-

i) Pour $\ell=0,1,2$ la suite $\left(u^{k}\right)$ est bornée dans l'espace

$$
C^{\ell}\left(\left[0,1\left[; H_{\mathrm{loc}}^{\frac{1}{2}-\ell}\left(Y, \mathbb{C}^{N}\right)\right)\right.\right.
$$

ii) Les traces $\left(u^{k}(0, y)\right),\left(\partial_{x} u^{k}(0, y)\right)$ convergent faiblement vers zéro respectivement dans $H_{\mathrm{loc}}^{1 / 2}\left(Y, \mathbb{C}^{N}\right)$ et $H_{\mathrm{loc}}^{-1 / 2}\left(Y, \mathbb{C}^{N}\right)$.

LEMme 2.3. - Pour tout opérateur pseudo-différentiel tangentiel, $Q\left(x, y, D_{y}\right)$, de degré strictement négatif, à support compact dans $\bar{X}$ (c'est-à-dire vérifiant $Q=\varphi Q \varphi$ pour un $\varphi \in$ $C^{\infty}(\bar{X})$ à support compact dans $\left.\bar{X}\right)$, la suite $\left(Q u^{k}\right)$ converge fortement vers zéro dans $H^{1}$.

LEMME 2.4. - Soit $A\left(x, y, D_{x}, D_{y}\right)$ un opérateur pseudo-différentiel sur $X$ de degré 0 , à support compact dans $X$ (c'est-à-dire vérifiant $A=\varphi A \varphi$ pour un $\varphi \in C_{0}^{\infty}(X)$ ), de symbole principal $a(x, y, \xi, \eta)$ nul sur l'ensemble $\left\{\xi^{2}=r(x, y, \eta)\right\}$. Alors la suite $\left(A u^{k}\right)$ converge fortement vers zéro dans $H^{1}(X)$.

Démonstration des lemmes 2.2, 2.3 et 2.4. - On remarque d'abord que $\left(u^{k}(x, y)\right)$ est bornée dans l'espace $E^{(3)}=\bigcap_{k=0}^{3} H^{k}(] 0,1\left[, H_{\text {loc }}^{1-k}(Y)\right)$, et converge faiblement vers zéro dans $E^{(3)}$, les cas $k=2,3$ résultant de l'équation (2.21) et de l'égalité $\partial_{x}^{2} u_{k}=v_{k}+R u_{k}$. Par les théorèmes de trace, il en résulte les points (i) et (ii) du lemme 2.2. Pour vérifier le lemme 2.3, il suffit de prouver que les suites $\left(Q \partial_{x} u^{k}\right)$ et $\left(Q \nabla_{y} u^{k}\right)$ convergent vers zéro fortement dans $L^{2}$. Or ces deux suites sont bornées dans l'espace $F=L^{2}(] 0,1\left[; H^{\varepsilon}(Y)\right) \cap H^{1}(] 0,1\left[; H^{-1+\varepsilon}(Y)\right)$ avec $\varepsilon=-\operatorname{deg}(Q)>0$ et l'injection de $F$ dans $L^{2}$ est compacte. Enfin, pour vérifier le lemme 2.4 de compacité intérieure nous noterons $z=(x, y), \zeta=(\xi, \eta)$ les variables cotangentes et $\Sigma_{[k]}^{m}$ l'espace des fonctions $a(z, \zeta)$ de classe $C^{\infty}$ et à support compact en $z$, de classe $C^{k-1}$ à dérivées $k^{\text {ième }} L^{\infty}$ en $\zeta$, et vérifiant

$$
\left|\partial_{z}^{\alpha} \partial_{\zeta}^{\beta} a(z, \zeta)\right| \leqslant C_{\alpha, \beta}(1+|\zeta|)^{m-|\beta|}
$$


pour tout $\alpha$ et tout $\beta$ vérifiant $|\beta| \leqslant k$ (les opérateurs pseudo-différentiels usuels correspondent à $k=\infty$ ). On quantifie le symbole $a$ par la règle usuelle

$$
\mathrm{Op}(a) f=(2 \pi)^{-(1+d)} \int \mathrm{e}^{i z \zeta} a(z, \zeta) \hat{f}(\zeta) \mathrm{d} \zeta
$$

Alors pour $a \in \Sigma_{[0]}^{m}, \operatorname{Op}(a)$ est borné de $H_{\text {comp }}^{s}$ dans $H_{\text {comp }}^{s-m}$ pour tout $s \in \mathbb{R}$, et pour $a \in \Sigma_{[1]}^{m}$, $b \in \Sigma_{[1]}^{m^{\prime}}$, on a

$$
\mathrm{Op}(a) \circ \mathrm{Op}(b)=\mathrm{Op}(a b)+\mathrm{Op}(c),
$$

avec $a b \in \Sigma_{[1]}^{m+m^{\prime}}$ et $c \in \Sigma_{[0]}^{m+m^{\prime}-1}$ (la preuve de ce résultat s'obtient très simplement en prolongeant les symboles qui sont à supports compacts en $z$ par périodicité sur une cellule assez grande, et en décomposant ces symboles en séries de Fourier, ce qui donne une décomposition de l'opérateur pseudo-différentiel en série de multiplicateurs de Fourier, la série étant à décroissance rapide). Soit $\varphi \in C_{0}^{\infty}(X)$.

On a $\varphi\left(-\xi^{2}+r(x, y, \eta)\right) \in \Sigma_{[2]}^{2}$ et

$$
\varphi\left(\partial_{x}^{2}+R\right)=\mathrm{Op}\left(\varphi\left(-\xi^{2}+r\right)\right)+\mathrm{Op}(d), \quad d \in \Sigma_{[1]}^{1} .
$$

Pour vérifier le lemme 2.4 , on peut, quitte à changer $A$ en un opérateur $A^{\prime}$, la différence $A-A^{\prime}$ étant régularisante, et à changer $a$ en $a^{\prime}$, supposer que $A=\operatorname{Op}(a) \circ \varphi$ et que la fonction $a(z, \zeta) \in \Sigma_{[\infty]}^{0}$ est à support compact en $z \in X$, contenu dans l'ensemble $\{\varphi \equiv 1\}$, et nulle au voisinage de $\{\zeta=0\}$. On a alors

$$
e:=\frac{a}{-\xi^{2}+r} \in \Sigma_{[2]}^{-2} .
$$

En effet la fonction $e(z, \zeta)$ est nulle au voisinage de $\zeta=0$, et est $C^{\infty}$ homogène de degré -2 en $\zeta$ pour $\zeta \neq 0$ puisque l'ensemble $S=\left\{-\xi^{2}+r=0\right\}$ est lisse pour $\zeta \neq 0$ d'après l'hypothèse 2.18, et $a$ s'annule sur $S$ (on utilise ici que si par exemple $\partial_{\eta_{d}} r \neq 0$ alors l'application

$$
(x, \xi, y, \eta) \mapsto\left(x, \xi, y, \eta_{1}, \ldots, \eta_{d-1},-\xi^{2}+r(x, y, \eta)\right)
$$

est un système de coordonnée local et dans ce système la fonction $a \in C^{\infty}$ s'annule sur l'ensemble $\zeta_{d}=0$ ce qui implique $\left.\frac{a}{\zeta_{d}} \in C^{\infty}\right)$; on a de plus $\left(-\xi^{2}+r\right)^{-1} \in \Sigma_{[2]}^{-2}$ dans un voisinage conique de $\eta=0$, par un calcul direct des dérivées d'ordre au plus 2 . On a

$$
\begin{aligned}
\varphi\left(\partial_{x}^{2}+R\right) \varphi u^{k} & =\varphi P \varphi u^{k}-\varphi\left(M_{0} \partial_{x}+M_{1}\right) \varphi u^{k} \\
& =\varphi P \varphi u^{k}+\operatorname{Op}(d) \varphi u_{k}:=w^{k},
\end{aligned}
$$

la suite $\left(w^{k}\right)$ est bornée dans $L^{2}$ et à support dans un compact fixe de $X$. Il suffit alors d'écrire les identités

$$
\begin{aligned}
& \mathrm{Op}(e) w^{k}=\mathrm{Op}(e)\left[\operatorname{Op}\left(\varphi\left(-\xi^{2}+r\right)\right) \varphi u^{k}+\mathrm{Op}(d) \varphi u^{k}\right], \\
& \operatorname{Op}(e)\left[w^{k}-\mathrm{Op}(d)\left(\varphi u^{k}\right)\right]=\mathrm{Op}(a) \varphi u^{k}+\mathrm{Op}(c) \varphi u^{k},
\end{aligned}
$$

où $c \in \Sigma_{[0]}^{-1}$, et de remarquer que les deux suites $\operatorname{Op}(c)\left[\varphi u^{k}\right]$ et $\operatorname{Op}(e)\left[w^{k}-\mathrm{Op}(d)\left(\varphi u^{k}\right)\right]$ sont bornées dans $H^{2}(X)$, donc convergent fortement vers zéro dans $H^{1}(X)$. 


\subsection{Construction de la mesure de défaut microlocale}

On note $\mathcal{A}$ l'espace des matrices $N \times N$ d'opérateurs $Q$ de la forme $Q=Q_{i}+Q_{\partial}$ où $Q_{i}$ est un opérateur pseudo-différentiel classique sur $X$, à support compact dans $X$ (i.e. vérifiant $Q_{i}=\varphi Q_{i} \varphi$ pour un $\left.\varphi \in C_{0}^{\infty}(X)\right)$ et où $Q_{\partial}$ est un opérateur pseudo-différentiel tangentiel classique à support compact dans $\bar{X}$ (i.e. vérifiant $Q_{\partial}=\varphi Q_{\partial} \varphi$ pour un $\varphi \in C^{\infty}(\bar{X})$ à support compact dans $\bar{X}$ ). On notera $\mathcal{A}^{s}$ les éléments de $\mathcal{A}$ qui sont de degré $s \in \mathbb{R}$, et $\mathcal{A}_{\text {sym }}$ les éléments de $\mathcal{A}^{0}$ dont le symbole principal est auto-adjoint. On décompose $T^{*} \partial X$ en l'union disjointe $\mathcal{E} \cup \mathcal{G} \cup \mathcal{H}$ où

$$
\mathcal{E}=\left\{r_{0}<0\right\}, \quad \mathcal{G}=\left\{r_{0}=0\right\}, \quad \mathcal{H}=\left\{r_{0}>0\right\} .
$$

On note ${ }^{b} T \bar{X}$ le fibré de rang $\operatorname{dim} X$ dont les sections sont les champs de vecteurs tangents à $\partial X$, ${ }^{b} T^{*} \bar{X}$ son fibré dual (le fibré cotangent compressé de Melrose) et $j: T^{*} \bar{X} \rightarrow{ }^{b} T^{*} \bar{X}$ l'application canonique. Le fibré ${ }^{b} T \bar{X}$ est engendré par les champs $\frac{\partial}{\partial y_{j}}, x \frac{\partial}{\partial x}$ et $j$ est définie par

$$
j(y, x, \eta, \xi)=(y, x, \eta, v=x \xi) .
$$

On note Car $P$ la variété caractéristique de $P$

$$
\operatorname{Car} P=\left\{(y, x, \eta, \xi) \in T^{*} \bar{X} ; \xi^{2}=r(x, y, \eta)\right\}
$$

et on pose

$$
Z=j(\operatorname{Car} P), \quad \hat{Z}=Z \cup j\left(\left.T^{*} \bar{X}\right|_{x=0}\right) .
$$

On a $\left.Z\right|_{x=0}=\left\{(y, 0, \eta, v=0), r_{0}(y, \eta) \geqslant 0\right\}$ et $\left.\hat{Z}\right|_{x=0}=\left.Z\right|_{x=0} \cup \mathcal{E}$. La fonction $r$ étant homogène de degré deux en $\eta$, il existe une constante $C$ telle que

$$
(y, x, \eta, v) \in \hat{Z} \Rightarrow|v| \leqslant C x|\eta| .
$$

Il en résulte que $\hat{Z}$ et $Z$ sont des fermés coniques de ${ }^{b} T^{*} \bar{X}$. On notera $S \hat{Z}$, et $S Z$ les espaces quotients sphériques

$$
S \hat{Z}=(\hat{Z} \backslash \bar{X}) / \mathbb{R}_{+}^{*}, \quad S Z=(Z \backslash \bar{X}) / \mathbb{R}_{+}^{*}
$$

qui sont des espaces métriques localement compacts. Pour $Q \in \mathcal{A}^{0}$, de symbole principal $q=\sigma(Q)$ on définit la fonction $\kappa(q) \in C^{0}\left(S \hat{Z} ; \operatorname{End}\left(\mathbb{C}^{N}\right)\right)$ par

$$
\kappa(q)(\rho)=q\left(j^{-1}(\rho)\right) .
$$

(C'est bien défini car $q$ est homogène, et on a $\kappa(q)(y, x, \eta, v)=q\left(y, x, \eta, \frac{v}{x}\right)$ pour $x \neq 0$ et $q$ est indépendant de $\xi$ pour $x$ assez petit.) D'après (2.35) l'ensemble

$$
\left\{\kappa(q), q=\sigma(Q), Q \in \mathcal{A}^{0}\right\}
$$

est localement dense dans $C^{0}\left(S \hat{Z} ; \operatorname{End}\left(\mathbb{C}^{N}\right)\right)$ où $C^{0}\left(S \hat{Z}, \operatorname{End}\left(\mathbb{C}^{N}\right)\right)$ est muni de la topologie de la convergence uniforme sur tout compact. Pour $Q \in \mathcal{A}^{0}, u \in H^{1}$ on pose

$$
\phi(Q, u)=(Q u \mid u)_{H^{1}}=\int_{X}\left(\nabla_{y} Q u \cdot \overline{\nabla_{y} u}+\partial_{x} Q u \cdot \overline{\partial_{x} u}+Q u \cdot \bar{u}\right) \mathrm{d} x \mathrm{~d} y .
$$


On notera $\mathcal{M}^{+}$l'espace des mesures boréliennes $\mu$ sur $S \hat{Z}$, à valeurs hermitiennes positives sur $\mathbb{C}^{N}$; une mesure $\mu$ de $\mathcal{M}^{+}$est donc un élément du dual de l'espace $C_{0}^{0}\left(S \hat{Z} ; \operatorname{End}\left(\mathbb{C}^{N}\right)\right)$ qui vérifie

$$
\langle\mu, q\rangle \geqslant 0, \quad \forall q \in C^{0}\left(S \hat{Z}, \operatorname{End}^{+}\left(\mathbb{C}^{N}\right)\right),
$$

où End ${ }^{+}\left(\mathbb{C}^{N}\right)$ désigne l'ensemble des matrices $N \times N$ hermitiennes positives.

Proposition 2.5. - Quitte à extraire une sous-suite de la suite $\left(u^{k}\right)$, il existe une mesure $\mu \in \mathcal{M}^{+}$telle que

$$
\forall Q \in \mathcal{A}^{0}, \quad \lim _{k \rightarrow \infty} \phi\left(Q, u^{k}\right)=\langle\mu, \kappa(\sigma(Q))\rangle .
$$

Démonstration. - On suit une démarche très classique inspirée de P. Gérard [5]. Remarquons d'abord que les lemmes 2.3 et 2.4 impliquent

$$
\forall Q \in \mathcal{A}^{-1}, \quad \lim _{k \rightarrow \infty} \phi\left(Q, u^{k}\right)=0 .
$$

Soit $\chi \in C_{0}^{\infty}(|x|<\varepsilon), 0 \leqslant \chi \leqslant 1, \chi(x)=1$ pour $|x| \leqslant \frac{\varepsilon}{2}$ et $E=E_{i}$ un opérateur scalaire pseudo-différentiel sur $X$ de degré 0 , à support près de Car $P$, vérifiant $0 \leqslant \sigma(E) \leqslant 1$ et égal à l'identité au voisinage de $\operatorname{Car} P \cap \operatorname{support}(1-\chi)$. D'après le lemme 2.4 , pour $\varphi \in C_{0}^{\infty}(X)$ on a

$$
(1-\chi)(\mathrm{Id}-E) \varphi u^{k} \rightarrow 0, \quad H^{1} \text { fort. }
$$

Si $Q=Q_{i}+Q_{\partial} \in \mathcal{A}^{0}$, en choisissant $\varepsilon$ assez petit on aura $\chi Q_{i} \equiv 0$ et on écrira

$$
Q=\chi Q+(1-\chi) Q=\chi Q_{\partial}+(1-\chi) Q E+(1-\chi) Q(\operatorname{Id}-E) .
$$

Alors $\chi Q_{\partial}$ est un o.p.d. tangentiel, $(1-\chi) Q E$ est un o.p.d. intérieur et

$$
(1-\chi) Q(\mathrm{Id}-E) \varphi u^{k} \rightarrow 0
$$

dans $H^{1}$ fort pour tout $\varphi \in C_{0}^{\infty}(\bar{X})$. Il en résulte pour $M \geqslant 0$

$$
\forall Q \in \mathcal{A}_{\mathrm{sym}}^{0}, \quad \sigma(Q) \geqslant-M \operatorname{Id} \Rightarrow \liminf _{k \rightarrow \infty} \phi\left(Q, u^{k}\right) \geqslant-M \limsup _{k \rightarrow \infty}\left\|u^{k}\right\|_{H^{1}}^{2} .
$$

En effet, $\sigma(Q) \geqslant-M$ Id implique

$$
\sigma\left(\chi Q_{\partial}\right)=\sigma(\chi Q) \geqslant-M \mathrm{Id} \quad \text { et } \quad \sigma((1-\chi) Q E)=(1-\chi) \sigma(E) \sigma(Q) \geqslant-M \mathrm{Id}
$$

et il suffit donc de traiter indépendamment les cas $Q=Q_{i}$ et $Q=Q_{\partial}$. Par exemple, dans le cas $Q=Q_{\partial}$, il existe $\varphi \in C_{0}^{\infty}(\bar{X})$ tel que

$$
\alpha_{k}=\left(\nabla Q_{\partial} u^{k} \mid \nabla u^{k}\right)_{L^{2}}=\left(Q_{\partial} \nabla\left(\varphi u^{k}\right) \mid \nabla\left(\varphi u^{k}\right)\right)_{L^{2}}+\beta_{k}
$$

avec $\beta_{k}=\left(\left[\nabla, Q_{\partial}\right] u^{k} \mid \nabla u^{k}\right)_{L^{2}} \rightarrow 0$. Pour tout $\eta>0$, il existe $B_{\partial}$ de degré $0, C_{\partial}$ de degré -1 , o.p.d. tangentiels tels que $Q_{\partial}+(M+\eta) \mathrm{Id}=B_{\partial}^{*} B_{\partial}+C_{\partial}$; comme $C_{\partial} \nabla\left(\varphi u^{k}\right) \rightarrow 0$ dans $L^{2}$ fort d'après le lemme 2.2 i), on a liminf $\alpha_{k} \geqslant-(M+\eta) \limsup \left\|\nabla \varphi u^{k}\right\|_{L^{2}}^{2}$ d'où (2.45).

On a aussi d'après le lemme 2.4 et (2.43)

$$
Q \in \mathcal{A}^{0}, \sigma(Q)=0 \text { près de } x=0 \text { et }\left.\sigma(Q)\right|_{\text {Car } P}=0 \Rightarrow \lim _{k} \phi\left(Q, u^{k}\right)=0 .
$$


Soit $\sigma\left(\mathcal{A}^{0}\right)=\left\{q=\sigma(Q), Q \in \mathcal{A}^{0}\right\} ;$ c'est un sous-espace vectoriel de l'espace des fonctions $C^{0}$ homogènes de degré zéro sur $T^{*} \bar{X} \backslash \bar{X}$ à valeur dans $\operatorname{End}\left(\mathbb{C}^{N}\right)$, muni de la norme $L^{\infty}$ et $\sigma\left(\mathcal{A}^{0}\right)$ possède une partie dense dénombrable. D'après les relations (2.42) et (2.45), quitte à extraire une sous-suite de la suite $\left(u^{k}\right)$, il existe une forme linéaire $\tilde{\phi}$ sur $\sigma\left(\mathcal{A}^{0}\right)$ telle que

$$
\begin{gathered}
\forall Q \in \mathcal{A}^{0}, \quad \lim _{k} \phi\left(Q, u^{k}\right)=\tilde{\phi}(\sigma(Q)), \\
|\tilde{\phi}(q)| \leqslant\|q\|_{L^{\infty}} \limsup \left\|u^{k}\right\|_{H^{1}}^{2} .
\end{gathered}
$$

On a de plus

$$
q \in \sigma\left(\mathcal{A}^{0}\right) \quad \text { et } \quad \kappa(q)=0 \Rightarrow \tilde{\phi}(q)=0
$$

car si $\kappa(q)=0$, pour tout $\varepsilon>0$, il existe $\chi \in C_{0}^{\infty}(\mathbb{R})$ à support près de $x=0$ tel que $\|\chi q\|_{L^{\infty}} \leqslant \varepsilon$ et $(1-\chi) q=\sigma(Q)$ où $Q \in \mathcal{A}^{0}$ vérifie (2.46). D'après (2.48) et (2.49) et le théorème de représentation de Riesz, il existe une mesure de Radon $\mu$, élément du dual de $C_{0}^{0}\left(S \hat{Z} ; \operatorname{End}\left(\mathbb{C}^{N}\right)\right)$ telle que

$$
\forall Q \in \mathcal{A}^{0}, \quad \lim _{k} \phi\left(Q, u^{k}\right)=\langle\mu, \kappa(\sigma(Q))\rangle
$$

et $\mu$ est hermitienne positive d'après (2.45).

On a $\left.\hat{Z}\right|_{x=0}=T^{*} Y$, et quitte à extraire une nouvelle sous-suite de la suite $\left(u^{k}\right)$, on construit de manière analogue des mesures sur $S\left(T^{*} Y\right)=\left(T^{*} Y \backslash Y\right) / \mathbb{R}_{+}^{*}, \mu_{\partial}^{1 / 2}, \mu_{\partial}^{-1 / 2}, \mu_{\partial}^{0}$ éléments du dual de $C^{0}\left(S\left(T^{*} Y\right)\right.$, End $\left.\left(\mathbb{C}^{N}\right)\right)$ caractérisées par

$$
\begin{gathered}
\forall Q \in \mathcal{A}^{0}, \quad \lim _{k} \int_{\partial X} Q u^{k} \cdot \overline{\partial_{x} u^{k}} \mathrm{~d} y=\left\langle\mu_{\partial}^{0},\left.\sigma(Q)\right|_{x=0}\right\rangle, \\
\forall Q \in \mathcal{A}^{1}, \quad \lim _{k} \int_{\partial X} Q u^{k} \cdot \overline{u^{k}} \mathrm{~d} y=\left\langle\mu_{\partial}^{1 / 2},\left.\frac{\sigma(Q)}{|\eta|}\right|_{x=0}\right\rangle, \\
\forall Q \in \mathcal{A}^{-1}, \quad \lim _{k} \int_{\partial X} Q \partial_{x} u^{k} \cdot \overline{\partial_{x} u^{k}} \mathrm{~d} y=\left\langle\mu_{\partial}^{-1 / 2},\left.|\eta| \sigma(Q)\right|_{x=0}\right\rangle .
\end{gathered}
$$

Les mesures $\mu_{\partial}^{1 / 2}$ et $\mu_{\partial}^{-1 / 2}$ sont hermitiennes positives, et l'inégalité de Cauchy-Schwarz entraîne

$$
\left|\left\langle\mu_{\partial}^{0}, a^{*} b\right\rangle\right|^{2} \leqslant\left\langle\mu_{\partial}^{1 / 2}, b^{*} b\right\rangle\left\langle\mu_{\partial}^{-1 / 2}, a^{*} a\right\rangle
$$

On posera

$$
\mu_{\partial}=\left(\begin{array}{cc}
\mu_{\partial}^{1 / 2} & \left(\mu_{\partial}^{0}\right)^{*} \\
\mu_{\partial}^{0} & \mu_{\partial}^{-1 / 2}
\end{array}\right),
$$

où $\left(\mu_{\partial}^{0}\right)^{*}$ est définie $\operatorname{par}\left\langle\left(\mu_{\partial}^{0}\right)^{*}, q\right\rangle=\overline{\left\langle\mu_{\partial}^{0}, q^{*}\right\rangle}$. Le lemme suivant prouve que $\mu$ détermine $\mu_{\partial}$.

LEMME 2.6.-

i) On $a$

$$
\mu 1_{\mathcal{H}}=0 \quad \text { et } \quad \mu_{\partial} 1_{\mathcal{G} \cup \mathcal{H}}=0 .
$$


ii) Soit $\theta$ la fonction sur $S \hat{Z}$ définie $\mu$ et $\mu_{\partial}$ presque partout d'après i), par

$$
\theta=\frac{\xi}{|\eta|} \quad \text { dans } x>0 ; \quad \theta=i \frac{\sqrt{-r_{0}(y, \eta)}}{|\eta|} \quad \text { sur } \mathcal{E} \cup \mathcal{G}
$$

On a les identités

$$
\begin{aligned}
\mu_{\partial}^{1 / 2} & =\frac{2 \theta}{i} \frac{1}{1+|\theta|^{2}} \mu 1_{x=0}, \\
\mu_{\partial}^{0} & =-\frac{2|\theta|^{2}}{1+|\theta|^{2}} \mu 1_{x=0}=-i \bar{\theta} \mu_{\partial}^{1 / 2}, \\
\mu_{\partial}^{-1 / 2} & =|\theta|^{2} \mu_{\partial}^{1 / 2} .
\end{aligned}
$$

Démonstration. - Pour $Q_{1} \in \mathcal{A}^{1}$ opérateur tangentiel, on a d'après ce qui précède

$$
\varlimsup\left\|Q_{1} u^{k}\right\|_{L^{2}} \leqslant \operatorname{Cte}\left|\frac{\sigma\left(Q_{1}\right)}{|\eta|}\right|_{L^{\infty}} \varlimsup\left\|u^{k}\right\|_{H^{1}} .
$$

Il en résulte pour $Q_{0} \in \mathcal{A}^{0}$ opérateur tangentiel

$$
\varlimsup\left\|Q_{0} \frac{\partial u^{k}}{\partial x}\right\|_{L^{2}}^{2} \leqslant \operatorname{Cte}\left|\frac{r \sigma\left(Q_{0}\right)}{|\eta|^{2}}\right|_{L^{\infty}}\left|\sigma\left(Q_{0}\right)\right|_{L^{\infty}} \varlimsup\left\|u^{k}\right\|_{H^{1}}^{2} .
$$

En effet, pour vérifier (2.60), on remarque que $\left[Q_{0}, \frac{\partial}{\partial x}\right] u^{k} \rightarrow 0$ dans $L^{2}$ fort et l'inégalité d'interpolation sur $\mathbb{R}_{+},\left|f^{\prime}\right|_{L^{2}}^{2} \leqslant C|f|_{L^{2}}\left|f^{\prime \prime}\right|_{L^{2}}$ implique, avec $E_{1}=\left(1-\Delta_{y}\right)^{1 / 2}$,

$$
\varlimsup\left\|\partial_{x} Q_{0} u^{k}\right\|_{L^{2}}^{2} \leqslant C \varlimsup \lim \left\|E_{1} Q_{0} u^{k}\right\|_{L^{2}} \varlimsup\left\|\partial_{x}^{2} E_{1}^{-1} Q_{0} u^{k}\right\|_{L^{2}}
$$

L'inégalité (2.60) résulte donc de (2.59) puisque l'on a

$$
\varlimsup\left\|E_{1}^{-1}\left[\partial_{x}^{2}, Q_{0}\right] u^{k}\right\|_{L^{2}}=0
$$

et

$$
\varlimsup\left\|E_{1}^{-1} Q_{0} \partial_{x}^{2} u^{k}\right\|_{L^{2}}=\varlimsup \lim \left\|E_{1}^{-1} Q_{0} R u^{k}\right\|_{L^{2}} .
$$

Soit $w$ un élément de l'espace $\bigcap_{k=0}^{2} H^{k}(] 0,1\left[; H^{1-k}(Y)\right)=E^{(2)}$ à valeurs dans $\mathbb{C}^{N}$ et à support compact dans $\bar{X}$. On obtient par intégration par parties

$$
(P u \mid w)_{L^{2}(X)}-\left(u \mid P^{*} w\right)_{L^{2}(X)}=\int_{\partial X}\left(u \cdot \partial_{x} \bar{w}-\partial_{x} u \cdot \bar{w}-u \cdot \overline{M_{0}^{*}(w)}\right) \mathrm{d} y .
$$

En appliquant (2.64) à $w=w^{k}=\left(Q_{0}+Q_{-1} \partial_{x}\right) u^{k}$ (qui appartient à $E^{(2)}$ ) avec $Q_{j} \in \mathcal{A}^{j}$, on obtient le membre de gauche de (2.64) convergeant vers zéro

$$
\left\langle\mu_{\partial}^{0}-\left(\mu_{\partial}^{0}\right)^{*}, \sigma\left(Q_{0}^{*}\right)\right\rangle=\left\langle\mu_{\partial}^{1 / 2}, \frac{\sigma\left(R Q_{-1}^{*}\right)}{|\eta|}\right\rangle+\left\langle\mu_{\partial}^{-1 / 2}, \sigma\left(Q_{-1}^{*}\right)|\eta|\right\rangle,
$$

ce qui prouve $\mu_{\partial}^{0}=\left(\mu_{\partial}^{0}\right)^{*}$ et

$$
\mu_{\partial}^{1 / 2} \frac{r_{0}}{|\eta|^{2}}+\mu_{\partial}^{-1 / 2}=0
$$

$4^{\text {e }}$ SÉRIE - TOME $34-2001-\mathrm{N}^{\circ} 6$ 
L'inégalité, pour $f \in H^{1}\left(\mathbb{R}_{+} \times \mathbb{R}_{y}^{d}\right)$

$$
\left\|\left.\left|\Delta_{y}\right|^{1 / 4} f\right|_{x=0}\right\|_{L^{2}\left(\mathbb{R}^{d}\right)}^{2} \leqslant \operatorname{Cte}\left\|\left|\Delta_{y}\right|^{1 / 2} f\right\|_{L^{2}\left(\mathbb{R}_{+} \times \mathbb{R}^{d}\right)}\left\|\partial_{x} f\right\|_{L^{2}\left(\mathbb{R}_{+} \times \mathbb{R}^{d}\right)}
$$

et (2.60) impliquent $\left.\mu_{\partial}^{-1 / 2}\right|_{\mathcal{G}}=0 ;$ (2.66) entraîne alors $\left.\mu_{\partial}^{ \pm 1 / 2}\right|_{\mathcal{G} \cup \mathcal{H}}=0$, donc aussi $\left.\mu_{\partial}^{0}\right|_{\mathcal{G} \cup \mathcal{H}}=0$ d'après (2.54), c'est-à-dire $\left.\mu_{\partial}\right|_{\mathcal{G} \cup \mathcal{H}}=0$.

Soit $\rho_{0}=\left(y_{0}, \eta_{0}\right) \in \mathcal{H}$; notons $\beta_{ \pm}=\left(y_{0}, x=0, \eta_{0}, \pm \sqrt{r_{0}\left(y_{0}, \eta_{0}\right)}\right)$ et $\gamma_{ \pm}$les deux demibicaractéristiques de $-\xi_{n}^{2}+r$ issues de $\beta^{ \pm}$. L'étude classique de la réflexion transverse nous apprend qu'il existe deux suites $u_{ \pm}^{k}$, bornées dans $H^{1}$, de limites faibles nulles, concentrées près de $\gamma_{ \pm}$, vérifiant $P u_{ \pm}^{k} \rightarrow 0$ dans $L^{2}$ et $\| Q_{0}\left(u^{k}-\left(u_{+}^{k}+u_{-}^{k}\right) \|_{H^{1}} \rightarrow 0\right.$ si $Q_{0} \in \mathcal{A}^{0}$ est à support proche de $\rho_{0}$. Comme $u_{ \pm}^{k}$ vérifient les équations pseudo-différentielles d'ordre 1 $\left(\frac{1}{i} \partial_{x}-Q_{ \pm}\right) u_{ \pm}^{k}=v_{ \pm}^{k}$ avec $v_{ \pm}^{k} \rightarrow 0$ dans $H^{1}$ et $\sigma\left(Q_{ \pm}\right)= \pm \sqrt{r} \mathrm{Id}$, les estimations d'énergie sur $\frac{1}{i} \partial_{x}-Q_{ \pm}$impliquent

$$
\left.u^{k}\right|_{x=0} \text { (resp. }\left.\partial_{x} u^{k}\right|_{x=0} \text { ) est borné dans } H^{1} \text { (resp. } L^{2} \text { ) près de } \rho_{0}
$$

et pour $\psi \in C_{0}^{\infty}, \lim _{\varepsilon \rightarrow 0} \varlimsup_{\lim _{k}}\left\|\psi\left(\frac{x}{\varepsilon}\right) Q_{1} u^{k}\right\|_{L^{2}}^{2}=0$ pour $Q_{1} \in \mathcal{A}^{1}$ à support près de $\rho_{0}$, d'où $\left.\mu\right|_{\mathcal{H}}=0$. Enfin, d'après le point i) et $\left.\theta\right|_{\mathcal{G}}=0$, il suffit de vérifier les relations (2.58) sur la région elliptique $\mathcal{E}$, où elles sont conséquences de la théorie elliptique standard.

Lemme 2.7. - Soient $Q_{i} \in \mathcal{A}^{i}$ des opérateurs tangentiels de degré $i=1,2$, de symboles principaux $q_{i}$. On a, avec $\lambda^{2}=|\eta|^{2}\left(1+\theta^{2}\right)$

$$
\lim _{k \rightarrow \infty}\left(\left(Q_{2}+Q_{1} \frac{1}{i} \partial_{x}\right) u^{k} \mid u^{k}\right)_{L^{2}(X)}=\left\langle\mu, \frac{\left(q_{2}+q_{1} \theta|\eta|\right)}{\lambda^{2}}\right\rangle .
$$

Démonstration. - On remarque d'abord que le membre de droite est bien défini car la fonction $\theta$ est définie $\mu$ presque partout. On peut supposer les $Q_{i}$ à support près de $\rho_{0} \in S \hat{Z}$. Lorsque $\rho_{0}$ est un point intérieur $\left(x\left(\rho_{0}\right)>0\right),(2.69)$ résulte de la construction de $\mu$. Si $\rho_{0}$ est un point du bord, on choisit une fonction $\varphi \in C_{0}^{\infty}(]-1,1[)$ égale à 1 au voisinage de $[-1 / 2,1 / 2]$ et pour $\varepsilon>0$ on écrit

$$
\begin{gathered}
Q_{i, \varepsilon}=(1-\varphi(x / \varepsilon)) Q_{i}(1-\varphi(x / 2 \varepsilon)), \\
Q_{i}^{\varepsilon}=Q_{i}-Q_{i, \varepsilon} .
\end{gathered}
$$

Alors puisque $Q_{i, \varepsilon}$ est supporté à l'intérieur,

$$
\lim _{k \rightarrow \infty}\left(\left(Q_{2, \varepsilon}+Q_{1, \varepsilon} \frac{1}{i} \partial_{x}\right) u^{k} \mid u^{k}\right)_{L^{2}(X)}=\left\langle\mu, \frac{(1-\varphi(x / 2 \varepsilon))\left(q_{2}+q_{1} \theta|\eta|\right)}{\lambda^{2}}\right\rangle
$$

et quand $\varepsilon$ tend vers 0 , si $Q$ est supporté près d'un point $\varrho_{0}$ hyperbolique, le terme de droite dans (2.71) tend vers

$$
\left\langle\mu 1_{x>0}, \frac{\left(q_{2}+q_{1} \theta|\eta|\right)}{\lambda^{2}}\right\rangle=\left\langle\mu, \frac{\left(q_{2}+q_{1} \theta|\eta|\right)}{\lambda^{2}}\right\rangle
$$

puisque $\mu 1_{\mathcal{H}}=0$. Il reste à montrer que la contribution de $Q_{i}^{\varepsilon}$ tend vers 0 quand $\varepsilon$ tend vers 0. On écrit (modulo des restes régularisants) $Q_{2}=-\Delta_{y} \operatorname{Op}\left(\frac{\tilde{q_{2}}}{|\eta|^{2}}\right)$ avec $\sigma\left(\tilde{q_{2}}\right)=\sigma\left(q_{2}\right)$ et $Q_{1}=\sum_{j=1}^{d} D_{y_{j}} \mathrm{Op}\left(q_{1, j}\right)$. On obtient alors après intégrations par parties (en $y$ ), 


$$
\begin{aligned}
& \left|\lim _{k \rightarrow \infty}\left(\left(Q_{2}^{\varepsilon}+Q_{1}^{\varepsilon} \frac{1}{i} \partial_{x}\right) u^{k} \mid u^{k}\right)_{L^{2}(X)}\right| \\
& \quad \leqslant\left|\left\langle\mu,(1-\varphi(x / \varepsilon)) \frac{q_{2}}{|\eta|^{2}}\right\rangle\right|+\sum_{j}\left|\left\langle\mu_{0, j},(1-\varphi(x / \varepsilon)) q_{j}\right\rangle\right|
\end{aligned}
$$

et le terme de droite tend vers 0 quand $\varepsilon$ tend vers 0 car $\mu 1_{\mathcal{H}}=0$. Pour $\rho_{0} \in \mathcal{E}$, (2.69) est conséquence de la théorie elliptique. Enfin pour $\rho_{0} \in \mathcal{G},(2.60)$ et $\left.\theta\right|_{\mathcal{G}}=0$ montrent que seul le cas $Q_{2}$ est à considérer. En reprenant la même décomposition de $Q_{2}$ que pour le cas hyperbolique on obtient que la contribution quand $\varepsilon$ tend vers 0 de $Q_{2, \varepsilon}$ tend vers

$$
\left\langle\mu 1_{\rho \notin(\mathcal{G} \cup \mathcal{E})}, \frac{q_{2}}{\lambda^{2}}\right\rangle \text {. }
$$

Pour étudier la contribution de $Q_{2}^{\varepsilon}$, on considère $\psi \in C_{0}^{\infty}(\mathbb{R})$ égale à 1 au voisinage de 0 et on note

$$
Q_{2}^{\varepsilon, \varepsilon^{\prime}}=O p\left(\psi\left(\frac{r_{0}(x, y, \eta)}{\varepsilon^{\prime}|\eta|^{2}}\right)\right) Q_{2}^{\varepsilon}
$$

$Q_{2}^{\varepsilon}-Q_{2}^{\varepsilon, \varepsilon^{\prime}}$ est donc supporté en dehors de $\mathcal{G}$, on peut donc lui appliquer ce qui précède. On obtient

$$
\lim _{\varepsilon, \varepsilon^{\prime} \rightarrow 0} \lim _{k \rightarrow \infty}\left(\left(Q_{2}^{\varepsilon}-Q_{2}^{\varepsilon, \varepsilon^{\prime}}\right) u^{k} \mid u^{k}\right)_{L^{2}(X)}=\left\langle\mu 1_{\mathcal{G}^{c}}, \frac{q_{2}}{\lambda^{2}}\right\rangle .
$$

Il reste à étudier la contribution de $Q_{2}^{\varepsilon, \varepsilon^{\prime}}$.

$$
\left(Q_{2}^{\varepsilon, \varepsilon^{\prime}} u^{k}, u^{k}\right)=\left(\left(-\Delta_{y}+1\right)\left(-\Delta_{y}+1\right)^{-1} Q_{2}^{\varepsilon, \varepsilon^{\prime}} u^{k}, u^{k}\right)
$$

On intègre par parties en $y$ et on remarque que d'après (2.60), la limite quand $\varepsilon, \varepsilon^{\prime}$ tendent vers 0 de $\lim _{k \rightarrow+\infty}\left(\partial_{x}\left(-\Delta_{y}+1\right)^{-1} Q_{2}^{\varepsilon, \varepsilon^{\prime}} u^{k}, \partial_{x} u^{k}\right)$ est nulle. On en déduit que le terme de droite dans (2.77) est égal à $\left\langle\mu, \frac{q_{2}^{\varepsilon, \varepsilon^{\prime}}}{|\eta|^{2}}\right\rangle+\mathrm{o}\left(\varepsilon+\varepsilon^{\prime}\right)$. Quand $\varepsilon, \varepsilon^{\prime}$ tendent vers 0 , on obtient que la contribution de $Q_{2}^{\varepsilon, \varepsilon^{\prime}}$ est

$$
\left\langle\mu 1_{\mathcal{G}}, \frac{q_{2}}{|\eta|^{2}}\right\rangle=\left\langle\mu 1_{\mathcal{G}}, \frac{q_{2}}{|\lambda|^{2}}\right\rangle .
$$

La contribution de $Q_{1}$ se traite de la même manière. On remarquera que dans ce raisonnement on prend garde à toujours faire tendre $k$ vers l'infini avant de faire tendre $\varepsilon, \varepsilon^{\prime}$ vers 0 .

\subsubsection{Condition aux limites}

Nous allons à présent travailler près d'un point $\rho_{0} \in T^{*} \partial X \backslash \partial X$

$$
\rho_{0}=\left(y_{0}, \eta_{0}\right) \in \mathcal{G}=\left\{r_{0}=0\right\} .
$$

Soit $s_{0} \in \mathbb{R}$ et $\left(f_{k}(y)\right)$ une suite bornée de $H_{\text {loc }}^{s_{0}}\left(Y, \mathbb{C}^{N}\right)$; pour $\alpha_{0} \in T^{*} Y \backslash Y$ et $s \in \mathbb{R}$, on dira que la suite $\left(f_{k}\right)$ est bornée dans $H_{\alpha_{0}}^{s}$ (resp. converge vers zéro fort dans $\left.H_{\alpha_{0}}^{s}\right)$ s'il existe un o.p.d. $E\left(y, D_{y}\right)$ de degré $-s$, elliptique en $\alpha_{0}$, à support compact dans $Y$, tel que la suite $\left(E f_{k}\right)$ soit bornée dans $L^{2}\left(Y, \mathbb{C}^{N}\right)$ (resp. converge vers zéro dans $L^{2}\left(Y, \mathbb{C}^{N}\right)$ ). Les mêmes propriétés restent alors valables pour tout o.p.d. $E$ à support compact dans $Y$ et dont le support essentiel 
est assez proche de $\alpha_{0}$. Nous supposerons dans la suite qu'il existe une matrice $B$, d'o.p.d. sur $Y$, de degré -1 , telle qu'on ait

$$
\left\{\begin{array}{l}
\left.u^{k}\right|_{x=0}=B\left(\left.\frac{1}{i}\left(\partial_{x} u^{k}\right)\right|_{x=0}\right)+h^{k}, \\
\left(h^{k}\right) \quad \text { converge vers zéro fort dans } H_{\rho_{0}}^{1} .
\end{array}\right.
$$

On notera $b$ le symbole principal de $B$. Près d'un point $\varrho_{0} \in \mathcal{G}$, la condition aux limites peut se mettre sous cette forme si et seulement si elle satisfait la condition de Lopatinski uniforme en $\rho_{0} \in \mathcal{G}$, voir [2, Chap VII.3].

LEMme 2.8. - Sous l'hypothèse (2.80), la suite $\left(\left.\left(\partial_{x} u^{k}\right)\right|_{x=0}\right)$ est bornée dans $L_{\rho_{0}}^{2}$. De plus, cette suite converge vers zéro fort dans $L_{\rho}^{2}$ pour $\rho \in \mathcal{E}$, proche de $\rho_{0}$.

Démonstration. - Soit $Q\left(x, y, D_{y}\right)$ un o.p.d. tangentiel de degré assez négatif et à support près de $x=0$. La suite $f^{k}=\left(\partial_{x}^{2}+R\right) u^{k}$ est bornée dans $L^{2}$ et $\left.f^{k}\right|_{x=0}$ est bornée dans $H_{\text {loc }}^{-1 / 2}\left(Y, \mathbb{C}^{N}\right)$. Par intégration par parties, on obtient, en tenant compte de $R^{*}=R$,

$$
\begin{aligned}
& \left(f^{k} \mid Q \partial_{x} u^{k}\right)_{L^{2}}+\left(\partial_{x} Q^{*} u^{k} \mid f_{k}\right)_{L^{2}}+\left.\int_{Y} Q^{*} u^{k} \cdot \bar{f}\right|_{x=0} \\
& \quad=\int_{Y}-\partial_{x} u^{k} \cdot \overline{Q \partial_{x} u^{k}}+u^{k} \cdot \overline{\partial_{x} Q \partial_{x} u^{k}}+\left(u,\left[\partial_{x}^{2}+R, Q \partial_{x}\right] u\right) .
\end{aligned}
$$

De plus, on a

$$
\left\{\begin{array}{l}
\left.\partial_{x} Q \partial_{x} u^{k}\right|_{x=0}=-\left.Q R u^{k}\right|_{x=0}+g^{k} \\
g^{k}=\left.\left[\partial_{x}, Q\right] \partial_{x} u^{k}\right|_{x=0}+Q\left[\left.v^{k}\right|_{x=0}-\left(\left.M_{0}\left(\partial_{x} u^{k}\right)\right|_{x=0}-\left.M_{1} u^{k}\right|_{x=0}\right] .\right.
\end{array}\right.
$$

Comme $\left(\left.u^{k}\right|_{x=0}\right)$ (resp. $\left.\left(\left.\partial_{x} u^{k}\right|_{x=0}\right)\right)$ est borné dans $H_{\text {loc }}^{1 / 2}\left(\right.$ resp. $\left.H_{\text {loc }}^{-1 / 2}\right)$, et $\left(\left.v^{k}\right|_{x=0}\right)$ est borné dans $H_{\text {loc }}^{-1 / 2}$, la suite $\left(g^{k}\right)$ est bornée dans $H_{\text {loc }}^{-1 / 2}\left(Y, \mathbb{C}^{N}\right)$, lorsque $Q$ est de degré 0 . On choisit $Q$ elliptique en $\rho_{0}$ vérifiant $Q+B^{*} Q R B=E^{*} E$, avec $E$ elliptique en $\rho_{0}, \chi$ un opérateur scalaire tangentiel d'ordre 0 à support proche de $\varrho_{0}$ et $\chi_{\varepsilon}$ une famille d'o.p.d. de degré $-\infty$ convergeant (au sens où les symboles convergent pour la topologie sur l'espace des symboles) vers $\chi$ et on pose $Q_{\varepsilon}=\chi_{\varepsilon}^{*} Q \chi_{\varepsilon}$ vérifiant $Q_{\varepsilon}+B^{*} Q_{\varepsilon} R B=\left(E \chi_{\varepsilon}\right)^{*}\left(E \chi_{\varepsilon}\right)+A_{-1, \varepsilon}$ où $A_{-1, \varepsilon}$ est une famille d'o.p.d. de degré $-\infty$ convergeant vers un opérateur d'ordre $-1, A_{-1}$. En appliquant (2.81) à la famille $Q_{\varepsilon}$, on obtient (puisque $\left[\partial_{x}^{2}+R, Q_{\varepsilon} \partial_{x}\right]$ est une famille uniformément bornée d'opérateurs de degré 2),

$$
\sup _{\varepsilon} \sup _{k}\left|\int_{Y} \partial_{x} u^{k} \cdot \overline{Q_{\varepsilon} \partial_{x} u^{k}}+u^{k} \cdot \overline{Q_{\varepsilon} R u^{k}}\right| \leqslant \operatorname{Cte}(Q),
$$

où $\operatorname{Cte}(Q)$ désigne une constante dépendant de $Q$. En utilisant à présent la condition aux limites (2.80), on obtient lorsque le support essentiel de $Q$ est proche de $\rho_{0}$

$$
\begin{aligned}
\int_{Y} \partial_{x} u^{k} \cdot \overline{Q_{\varepsilon} \partial_{x} u^{k}}+u^{k} \cdot \overline{Q_{\varepsilon} R u^{k}} \\
=\left(\partial_{x} u^{k} \mid\left(Q_{\varepsilon}+B^{*} Q_{\varepsilon} R B\right) \partial_{x} u^{k}\right)_{L^{2}\left(Y ; \mathbb{C}^{N}\right)} \\
\quad+\left(h^{k}, Q_{\varepsilon} R h^{k}\right)+\left(h^{k}, Q_{\varepsilon} R B\left(\frac{\partial x}{i} u^{k}\right)\right)+\left(B\left(\frac{\partial_{x}}{i} u^{k}\right), Q_{\varepsilon} R h^{k}\right),
\end{aligned}
$$


ce qui implique, puisque $\left(h^{k}\right)$ est bornée dans $H_{\varrho_{0}}^{1}$

$$
\left\|E \chi_{\varepsilon} \partial_{x} u^{k}\right\|^{2} \leqslant C(Q, \chi)\left(1+\left\|E \chi_{\varepsilon} \partial_{x} u^{k}\right\|\right),
$$

soit

$$
\left\|E \chi_{\varepsilon} \partial_{x} u^{k}\right\| \leqslant C^{\prime}(Q, \chi) \text {. }
$$

Passant à la limite $\varepsilon \rightarrow 0$, on obtient le résultat souhaité. Le fait que la suite $\left.\left(\partial_{x} u^{k}\right)\right|_{x=0}$ converge vers zéro fort dans $L_{\rho}^{2}$ pour $\rho \in \mathcal{E}$ proche de $\rho_{0}$ résulte de (2.80) et de la théorie elliptique standard.

On notera $\nu$ la mesure hermitienne positive associée à la suite bornée $\left(\left.\partial_{x} u^{k}\right|_{x=0}\right)$ de $L_{\rho_{0}}^{2}$ (qui est de limite faible nulle d'après le lemme 2.2), après extraction éventuelle d'une sous-suite. Si $A\left(y, D_{y}\right)$ est un o.p.d. de degré 0 matriciel à support compact dans $Y$ et à support essentiel proche de $\rho_{0}$, on a donc

$$
\lim _{k \rightarrow \infty} \int_{Y}\left(A \partial_{x} u_{k}\right) \cdot \overline{\partial_{x} u_{k}}=\langle\nu, \sigma(A)\rangle,
$$

où $\sigma(A)$ est le symbole principal de $A$ qui appartient à $C^{\infty}\left(S\left(T^{*} Y\right), \operatorname{End}\left(\mathbb{C}^{N}\right)\right)$ et est à support proche de $\rho_{0}$. La mesure $\nu$ est portée par $\mathcal{G} \cup \mathcal{H}$ d'après le lemme 2.8.

LEMME 2.9. - Pour $i=0,1$, soient $Q_{i}\left(x, y, D_{y}\right)$ des o.p.d.tangentiels matriciels de degré $i$, à support compact proche de $(y, \eta)=\rho_{0}, x=0$, et $Q=Q_{0} \frac{1}{i} \partial_{x}+Q_{1}$. On a

$$
\lim _{k \rightarrow \infty}\left(i\left[P^{*} Q-Q P\right] u^{k} \mid u^{k}\right)_{L^{2}(X)}=\left\langle\nu, q_{0}+b^{*} q_{0} r_{0} b+q_{1} b+b^{*} q_{1}\right\rangle,
$$

avec $q_{i}=\sigma\left(Q_{i}\right)(y, \eta, x=0)$.

Démonstration. - Rappelons qu'on a $u^{k} \in \bigcap_{\ell=0}^{3} H^{\ell}(] 0,1\left[; H_{\mathrm{loc}}^{1-\ell}(Y)\right)$ et $P u^{k}=v^{k}$, où $v^{k}$ tend fortement vers 0 dans $\bigcap_{\ell=0}^{1} H^{\ell}(] 0,1\left[, H_{\text {loc }}^{-\ell}(Y)\right)$. Soit $\varphi \in C_{0}^{\infty}(\bar{X})$, égale à 1 au voisinage du support des $Q_{i}$ et $u_{\varphi}^{k}=\varphi u^{k}$. Par intégration par parties, on obtient

$$
\left(P u^{k} \mid Q^{*} u_{\varphi}^{k}\right)_{L^{2}(X)}=\left(Q P u^{k} \mid u_{\varphi}^{k}\right)_{L^{2}(X)}-i \int_{Y} v^{k} \cdot \overline{Q_{0}^{*} u_{\varphi}^{k}},
$$

où par convention la dualité $(\alpha \mid \beta)_{L^{2}(X)}$ s'applique à $\alpha \in L^{2}(] 0,1\left[, H^{s}(Y)\right)$ et $\beta \in L^{2}(] 0,1[$, $\left.H^{-s}(Y)\right)$ avec $\alpha$ ou $\beta$ à support compact et $s \in \mathbb{R}$. De même, si les $Q_{i}$ sont de degré assez négatif, on obtient par intégration par parties

$$
\begin{aligned}
& \left(Q u^{k} \mid P u_{\varphi}^{k}\right)_{L^{2}(X)} \\
& \quad=\left(P^{*} Q u^{k} \mid u_{\varphi}^{k}\right)_{L^{2}(X)}+\int_{Y}-Q u^{k} \cdot \overline{\partial_{x} u_{\varphi}^{k}}+\partial_{x} Q u^{k} \cdot \overline{u_{\varphi}^{k}}-M_{0}^{*}\left(Q u^{k}\right) \cdot \overline{u_{\varphi}^{k}} .
\end{aligned}
$$

En retranchant (2.89) à (2.90), on obtient, pour des opérateurs $Q^{i}$ de degré assez négatif

$$
\begin{aligned}
\left(Q u^{k} \mid P u_{\varphi}^{k}\right)_{L^{2}(X)}-\left(P u^{k} \mid Q^{*} u_{\varphi}^{k}\right)_{L^{2}(X)} & \\
= & \left(\left(P^{*} Q-Q P\right) u^{k} \mid u_{\varphi}^{k}\right)_{L^{2}(X)} \\
& +\int_{Y}-Q u^{k} \cdot \overline{\partial_{x} u_{\varphi}^{k}}+\partial_{x} Q u^{k} \cdot \overline{u_{\varphi}^{k}}-M_{0}^{*}\left(Q u^{k}\right) \cdot \overline{u_{\varphi}^{k}}+i v^{k} \cdot \overline{Q_{0}^{*} u_{\varphi}^{k}} .
\end{aligned}
$$


Lorsque les opérateurs $Q_{i}$ sont de degré $i$, on a $\left(P^{*} Q-Q P\right)=\sum_{j=0}^{2} A_{2-j} \partial_{x}^{j}$ où les $A_{\ell}$ sont des opérateurs tangentiels de degré $\ell$, donc $\varphi\left(P^{*} Q-Q P\right) u^{k}$ appartient à $L^{2}(] 0,1\left[, H^{-1}(Y)\right)$ et est à support compact dans $\bar{X}$. Si de plus les $Q_{i}$ sont à support proche de $\rho_{0}$, on a d'après le lemme $2.8,\left.Q u^{k}\right|_{x=0} \in L^{2}$ et

$$
\begin{aligned}
\left.\partial_{x} Q u^{k}\right|_{x=0}= & \left.\frac{\partial Q_{0}}{\partial x} \frac{1}{i} \partial_{x} u^{k}\right|_{x=0}+\left.\frac{\partial Q_{1}}{\partial x} u^{k}\right|_{x=0}+\left.Q_{1} \partial_{x} u^{k}\right|_{x=0} \\
& +\left.Q_{0} \frac{1}{i} v^{k}\right|_{x=0}+\left.Q_{0} \frac{1}{i}\left[-R u^{k}-M_{0} \partial_{x} u^{k}-M_{1} u^{k}\right]\right|_{x=0},
\end{aligned}
$$

donc $\left.\partial_{x} Q u^{k}\right|_{x=0}$ appartient à $H^{-1}(Y)$. L'identité (2.91) reste donc valable pour $Q$ vérifiant les hypothèses du lemme 2.9. En faisant tendre $k$ vers l'infini dans l'identité (2.91), on obtient alors (2.88) en utilisant la condition aux limites (2.80) et la définition (2.87) de la mesure $\nu$.

Pour $\rho$ proche de $\rho_{0} \in T^{*} Y$, et $\xi$ proche de zéro, on note $J_{\xi}(\rho)$ l'endomorphisme de $\mathbb{C}^{N}$ défini par

$$
J_{\xi}(\rho)=(\operatorname{Id}-\xi b(\rho))^{-1}(\operatorname{Id}+\xi b(\rho)),
$$

où $b(\rho)$ est le symbole principal de $B$. On remarquera que $J_{0}(\rho)=$ Id et $J_{-\xi}(\rho)=\left[J_{\xi}(\rho)\right]^{-1}$. L'endomorphisme $-J_{\xi}(\rho)$, pour $\rho \in \mathcal{H}$ décrit la réflexion hyperbolique associée à la condition aux limites (2.80) entre les points $(\rho,-\xi)$ et $(\rho, \xi)$. Nous noterons également $C_{\text {paire }}^{\infty, k}\left(p^{-1}(0)\right.$, $\left.\operatorname{End}\left(\mathbb{C}^{N}\right)\right)$ le sous-espace des fonctions $q C^{\infty}$ homogènes de degré $k$ de $p^{-1}(0)$ à valeurs dans $\operatorname{End}\left(\mathbb{C}^{N}\right)$, à support près de $x=0, \xi=0,(y, \eta)=\rho_{0}$ et qui vérifient la condition de symétrie (pour $\rho$ proche de $\rho_{0}$ )

$$
\forall \xi, \quad J_{\xi}^{*}(\rho) q(x=0, \xi, \rho) J_{\xi}(\rho)=q(x=0,-\xi, \rho) .
$$

Soit $\mu$ la mesure associée à la suite $\left(u^{k}\right)$. On a $\left.\mu\right|_{\mathcal{E}}=0$ près de $\rho_{0}$ car d'après le lemme 2.8 , les suites $\left.u^{k}\right|_{x=0}$ et $\left.\partial_{x} u^{k}\right|_{x=0}$ convergent vers zéro fort dans $H_{\rho_{0}}^{1 / 2}$ et $H_{\rho_{0}}^{-1 / 2}$. Comme $\left.\mu\right|_{\mathcal{H}}=0$, on peut à présent considérer $\mu$ comme une mesure sur $p^{-1}(0) / \mathbb{R}_{+}^{*}$, c'est-à-dire comme un élément du dual de l'espace $C_{0}^{0}\left(p^{-1}(0) / \mathbb{R}_{+}^{*} ; \operatorname{End}\left(\mathbb{C}^{N}\right)\right)$ via l'application canonique

$$
p^{-1}(0) \backslash 0 / \mathbb{R}_{+}^{*} \stackrel{j}{\rightarrow} S Z
$$

qui est un homéomorphisme de $\left(p^{-1}(0) \backslash\{x=0, \xi \neq 0\}\right) / \mathbb{R}_{+}^{*}$ sur $S Z \backslash \mathcal{H}$.

LEMME 2.10. - Soient

$$
m(x, \xi, \rho)=m_{0}(x, \rho) i \xi+m_{1}(x, \rho)
$$

et

$$
q \in C_{\text {paire }}^{\infty, 1}\left(p^{-1}(0), \operatorname{End}\left(\mathbb{C}^{N}\right)\right)
$$

La mesure $\mu$ associée à la suite $\left(u^{k}\right)$ (qui satisfait la condition aux limites de la relation (2.80)) vérifie l'équation

$$
\left\langle\mu, \frac{\{p, q\}+i\left(m^{*} q-q m\right)}{\lambda^{2}}\right\rangle=0 .
$$


Démonstration. - Soit $q \in C_{0}^{\infty}\left(p^{-1}(0), \operatorname{End}\left(\mathbb{C}^{N}\right)\right)$, homogène de degré 1 , à support près de $\left(x=0, \xi=0, \rho_{0}\right)$. D'après le théorème de division de Malgrange (voir [7, Chap VII.7.5]), il existe des opérateurs tangentiels $Q_{i}$ de degré $i, i=0,1$, de symboles principaux $q_{i}$ tels que

$$
q=\left.\left(q_{0} \xi+q_{1}\right)\right|_{p^{-1}(0)}
$$

et on peut choisir les $Q_{i}$ à support compact dans $\bar{X}$ et à support essentiel proche de $x=0, \rho=\rho_{0}$. En notant $Q=Q_{0} \frac{1}{i} \partial_{x}+Q_{1}$ et $M=M_{0} \partial_{x}+M_{1}$, on a

$$
\left\{\begin{aligned}
i\left(P^{*} Q-Q P\right) & =i\left[\partial_{x}^{2}+R, Q\right]+i\left(M^{*} Q-Q M\right), \\
& =A_{2}+A_{1} \frac{1}{i} \partial_{x}+A_{0} P,
\end{aligned}\right.
$$

où les $A_{j}$ sont des opérateurs à supports compacts, tangentiels matriciels de degré $j$, de symboles principaux $a_{j}$. On a donc l'égalité sur $p^{-1}(0) \backslash \mathcal{H}$ (avec $\theta$ définie par (2.57))

$$
a_{2}+a_{1} \theta|\eta|=\{p, q\}+\left.i\left(m^{*} q-q m\right)\right|_{p^{-1}(0)} .
$$

D'après les lemmes 2.6, 2.7 et 2.8, l'identité (2.98) est donc conséquence de (2.101) si nous vérifions pour $q \in C_{\text {paire }}^{\infty, 1}\left(p^{-1}(0), \operatorname{End}\left(\mathbb{C}^{N}\right)\right)$ l'identité

$$
\left.\left(q_{0}+b^{*} q_{0} r b+q_{1} b+b^{*} q_{1}\right)\right|_{(x=0) \backslash \mathcal{E}} \equiv 0 .
$$

D'après (2.94) et (2.99), on a sur $\{x=0\} \cap p^{-1}(0)$

$$
J_{\xi}^{*} \circ\left(q_{0} \xi+q_{1}\right) \circ J_{\xi}=-q_{0} \xi+q_{1} .
$$

D'après (2.93), cela signifie que sur $x=0 \cap p^{-1}(0)$, la fonction matricielle $\xi \mapsto\left(\operatorname{Id}-\xi b^{*}\right)^{-1}$ $\left(q_{0} \xi+q_{1}\right)(\mathrm{Id}-\xi b)^{-1}$ est paire en $\xi$; ceci équivaut à dire que sur $\{x=0\} \cap p^{-1}(0)=\mathcal{G} \cup \mathcal{H}$, la fonction matricielle $\xi \mapsto\left(\operatorname{Id}+\xi b^{*}\right)\left(q_{0} \xi+q_{1}\right)(\mathrm{Id}+\xi b)$ est paire en $\xi$, ce qui entraîne $\left.\left(q_{0}+b^{*} q_{0} r b+q_{1} b+b^{*} q_{1}\right)\right|_{\mathcal{H}}=0$, donc (2.102) par continuité puisque $\mathcal{G} \subset \overline{\mathcal{H}}$.

\section{Mesures invariantes}

Dans ce paragraphe, nous noterons $\Sigma$ un petit voisinage (pour que sur $\Sigma$ (2.80) soit satisfaite), conique dans $Z=j(\operatorname{Car} P)$ du point $\rho_{0}=\left(y_{0}, \eta_{0}\right) \in \mathcal{G}$, de sorte que la condition aux limites (2.80) soit satisfaite près de $\bar{\Sigma}$ (et $|\xi b(\rho)|<1$ sur $\Sigma$ ). Près de $\rho_{0}$, on a donc

$$
\left\{\begin{array}{l}
\Sigma \cap(x>0)=\left\{(y, \eta, x, \xi) ; \xi^{2}=r(x, y, \eta)\right\}, \\
\Sigma \cap(x=0)=\left\{(y, \eta) ; r_{0}(y, \eta) \geqslant 0\right\} .
\end{array}\right.
$$

L'espace $\Sigma$ est un espace métrique localement compact. On remarquera que puisque $\rho_{0}$ est le projeté d'un point de la variété caractéristique vérifiant $\xi_{0}=0$, on a $\eta_{0} \neq 0$.

\subsection{Le flot de Melrose-Sjöstrand}

On notera $\pi$ la projection canonique de Car $P=p^{-1}(0)=\left\{\xi^{2}=r(x, y, \eta)\right\}$ sur $\Sigma$. L'ensemble $\Sigma$ est stratifié par

$$
\Sigma=\bigcup_{j=0}^{\infty} \Sigma^{j}
$$


où

$$
\begin{aligned}
\Sigma^{0} & =\Sigma \cap(x>0), \\
\Sigma^{1} & =\mathcal{H}=\left\{(y, \eta) ; r_{0}(y, \eta)>0\right\}, \\
\Sigma^{2} & =\left\{(y, \eta) ; r_{0}(y, \eta)=0, r_{1}(y, \eta) \neq 0\right\}, \\
\cdots & \\
\Sigma^{k+3} & =\left\{(y, \eta) ; r_{0}(y, \eta)=0, H_{r_{0}}^{j}\left(r_{1}\right)=0, \forall j \leqslant k ; H_{r_{0}}^{k+1}\left(r_{1}\right) \neq 0\right\}, \\
\cdots & \\
\Sigma^{\infty} & =\left\{(y, \eta) ; r_{0}(y, \eta)=0, H_{r_{0}}^{j}\left(r_{1}\right)=0, \forall j\right\} .
\end{aligned}
$$

On a $\mathcal{G}=\left\{r_{0}=0\right\}=\bigcup_{j \geqslant 2} \Sigma^{j}$, et on décompose $\Sigma^{2}$ en l'union disjointe

$$
\Sigma^{2}=\mathcal{G}^{2,+} \cup \mathcal{G}^{2,-}, \quad \mathcal{G}^{2, \pm}=\left\{(y, \eta) \in \Sigma^{2}, \pm r_{1}(y, \eta)>0\right\}
$$

Les points de $\mathcal{G}^{2,+}$ sont les points strictement diffractifs, et ceux de $\mathcal{G}^{2,-}$ les points strictement glissants. Les point de $\Sigma^{1}$ sont ceux en lesquels la bicaractéristique de $p$ est transverse au bord $x=0$ (points hyperboliques). Les points de $\Sigma^{j}, j \geqslant 2$, sont ceux en lesquels la bicaractéristique de $p$ a un contact d'ordre $j$ avec le bord $x=0$. La fonction $p=-\xi^{2}+r(x, y, \eta)$ étant homogène de degré 2 sur le fibré cotangent, le flot de son champ hamiltonien, $H_{p}$, n'induit pas un flot sur l'espace quotient homogène $\operatorname{Car} P / \mathbb{R}_{+}^{*}$. Pour remédier à ce fait, on reparamètre les bicaractéristiques de $p$ en choisissant une fonction $\tau(y, \eta)$, homogène de degré 1 positive, $C^{\infty}$ et ne s'annulant pas sur $\Sigma$ (sauf pour $\eta=0$ ). Les champs $H_{p}$ et $\frac{1}{\tau} H_{p}$ ont les mêmes courbes intégrales, et sur $p^{-1}(0)$, on a $\frac{1}{\tau} H_{p}=H_{p / \tau}$. Le paramètre $s$ que nous choisirons sur les bicaractéristiques généralisées sera le paramètre naturel pour le champ $\frac{1}{\tau} H_{p}$. Rappelons qu'un rayon est une application continue d'un intervalle $I$ de $\mathbb{R}$ dans $\Sigma, s \mapsto \gamma(s)$, qui vérifie en tout point $\rho_{1}=\gamma\left(s_{1}\right)$.

i) Si $\rho_{1} \in \mathcal{H} \cup \mathcal{G}^{2,+}$ : il existe $\varepsilon>0$ tel que l'on ait $x(\gamma(s))>0$ pour $0<\left|s-s_{1}\right|<\varepsilon$.

ii) Si $\rho_{1} \notin \mathcal{H} \cup \mathcal{G}^{2,+}\left(\pi^{-1}\left(\rho_{1}\right)\right.$ est alors un singleton dans $\left.p^{-1}(0)\right)$ : pour toute application $f$ $C^{\infty}$ de $p^{-1}(0)$ dans $\mathbb{R}$, et $\pi$-invariante, si on note $\tilde{f}$ l'unique application continue de $\Sigma$ dans $\mathbb{R}$ qui rend commutatif le diagramme

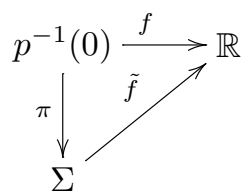

la fonction $s \mapsto \tilde{f}[\gamma(s)]$ est dérivable en $s=s_{1}$ et on a

$$
\frac{\mathrm{d}}{\mathrm{d} s}(\tilde{f} \circ \gamma)\left(s_{1}\right)=\frac{1}{\tau} H_{p}(f)\left[\pi^{-1}\left(\rho_{1}\right)\right] .
$$

Dans toute la suite, on supposera qu'il n'y a pas de contact d'ordre infini entre les bicaractéristiques de $p$ et le bord $x=0$, c'est-à-dire

$$
\exists J, \quad \Sigma=\bigcup_{j \leqslant J} \Sigma^{j} .
$$


Sous l'hypothèse (3.5), R. Melrose et J. Sjöstrand ont prouvé [14] que par tout point $\rho_{1}$ de $\Sigma$ il existe un unique rayon maximal $s \in] a, b\left[\stackrel{\gamma}{\longrightarrow} \Sigma\right.$ vérifiant $\gamma(0)=\rho_{1}$ et $\gamma(a+0) \cup \gamma(b-0) \in$ $\bar{\Sigma} \backslash \Sigma$.

Dans la suite on notera $\phi(s, \rho)$ le flot de Melrose-Sjöstrand ainsi défini, vérifiant $\phi(0, \rho)=\rho$. Le flot $\phi$ est homogène pour l'action de $\mathbb{R}_{+}^{*}$ sur $\Sigma$, c'est-à-dire vérifie $\phi(s, t \rho)=t \cdot \phi(s, \rho)$, donc induit un flot, qu'on notera aussi $\phi$ sur le quotient $\Sigma / \mathbb{R}_{+}^{*}$.

\subsection{Trivialisation du fibré le long du flot}

On note $\rho=(y, \eta)$ le point courant de $T^{*} Y \simeq T^{*} \partial X$ et $\mathcal{J}$ le morphisme de fibré vectoriel hermitien, $\mathbb{C}^{N}$ étant muni de son produit scalaire usuel

$$
\left\{\begin{array}{l}
p^{-1}(0) \times \mathbb{C}^{N} \stackrel{\mathcal{J}}{\longmapsto} p^{-1}(0) \times \mathbb{C}^{N} \\
\{(\rho, x, \xi) ; e\} \longmapsto(\rho, x, \xi) ;\left[J_{\xi}(\rho)\right]^{+1 / 2}(e),
\end{array}\right.
$$

où $J_{\xi}(\rho)$ est l'endomorphisme de $\mathbb{C}^{N}$ défini en (2.93) et

$$
\left[J_{\xi}(\rho)\right]^{+1 / 2}=\exp \left(\frac{1}{2} \log J_{\xi}(\rho)\right) .
$$

Le morphisme $\mathcal{J}$ est bien défini près de $\left(\rho_{0}, 0,0\right)$ et on a $\left.\mathcal{J}\right|_{\mathcal{G}}=\operatorname{Id}$. Si $q \in C_{0}^{\infty}\left(p^{-1}(0)\right.$, $\left.\operatorname{End}\left(\mathbb{C}^{N}\right)\right)$ est considérée comme forme hermitienne par le produit scalaire de $\mathbb{C}^{N}$, son image inverse par $\mathcal{J}$ notée $\tilde{q}$ est définie par

$$
\left(\tilde{q}_{\rho, x, \xi}(e) \mid e\right)=\left(\left(J_{\xi}^{* 1 / 2}(\rho) q_{\rho, x, \xi} J_{\xi}^{1 / 2}(\rho)\right)(e) \mid e\right) .
$$

On notera $\tilde{q}_{\xi}=J_{\xi}^{* 1 / 2} q_{\xi} J_{\xi}^{1 / 2}$ pour simplifier les notations. Par définition, on a $q \in C_{\text {paire }}^{\infty}\left(p^{-1}(0)\right.$, $\left.\operatorname{End}\left(\mathbb{C}^{N}\right)\right)$ si et seulement si $q \in C_{0}^{\infty}\left(p^{-1}(0), \operatorname{End}\left(\mathbb{C}^{N}\right)\right)$ et $J_{\xi}^{*} q_{\xi} J_{\xi}=q_{-\xi}$ sur le bord $x=0$, ce qui équivaut à $\tilde{q}_{-\xi}=\tilde{q}_{\xi}$ sur le bord $x=0$, puisque $J_{-\xi}=J_{\xi}^{-1}$. Si $\mu$ est la mesure du lemme 2.10, on définit la mesure hermitienne positive $\tilde{\mu}$, élément du dual de $C_{0}^{0}\left(p^{-1}(0) / \mathbb{R}_{+}^{*}, \operatorname{End}\left(\mathbb{C}^{N}\right)\right)$ par

$$
\langle\tilde{\mu}, \tilde{q}\rangle=\langle\mu, q\rangle, \quad \tilde{q}_{\xi}=J_{\xi}^{* 1 / 2} q_{\xi} J_{\xi}^{1 / 2} .
$$

En d'autres termes, $\tilde{\mu}$ est l'image inverse de $\mu$ par l'isomorphisme $\mathcal{J}$. Si on note $C_{+}^{\infty, k}\left(p^{-1}(0) / \mathbb{R}_{+}^{*}, \operatorname{End}\left(\mathbb{C}^{N}\right)\right)$ l'espace des sections $C^{\infty} \tilde{q}$ homogènes de degré $k$ de $p^{-1}(0) / \mathbb{R}_{+}^{*}$ à valeurs dans $\operatorname{End}\left(\mathbb{C}^{N}\right)$, à support compact, et qui vérifient $\tilde{q}(\rho, 0, \xi)=\tilde{q}(\rho, 0,-\xi)$, le lemme 2.10 est équivalent à

$$
\begin{aligned}
& \left\langle\tilde{\mu}, \frac{\{p, \tilde{q}\}+i\left(n^{*} \tilde{q}-\tilde{q} n\right)}{\lambda^{2}}\right\rangle=0, \quad \forall \tilde{q} \in C_{+}^{\infty, 1}\left(p^{-1}(0), \operatorname{End}\left(\mathbb{C}^{N}\right)\right) \\
& \text { avec } n=J^{-1 / 2} m J^{1 / 2}+i\left\{p, J^{-1 / 2}\right\} J^{1 / 2}
\end{aligned}
$$

On remarquera que le passage de la mesure $\mu$ à la mesure $\tilde{\mu}$ correspond exactement à un changement de structure hermitienne sur le fibré $p^{-1}(0) \times \mathbb{C}^{N}$, qui permet de ramener le morphisme d'identification $J_{\xi}(e)$ de la réflexion hyperbolique à l'identité; les équations (2.98) et (3.9) vérifiées par les mesures $\mu$ et $\tilde{\mu}$ sont formellement identiques. Si $\Sigma \times \mathbb{C}^{N}$ est le fibré hermitien trivial sur $\Sigma, \mathbb{C}^{N}$ étant muni de son produit scalaire usuel, on notera $\hat{\mu}$ la mesure hermitienne positive élément du dual de $C_{0}^{0}\left(\Sigma / \mathbb{R}^{*} ; \operatorname{End}\left(\mathbb{C}^{N}\right)\right)$ définie par

$$
\langle\hat{\mu}, q\rangle=\langle\tilde{\mu}, q \circ \pi\rangle,
$$

$4^{\text {e }}$ SÉRIE - TOME $34-2001-\mathrm{N}^{\circ} 6$ 
où $\pi: p^{-1}(0) / \mathbb{R}_{+}^{*} \rightarrow \Sigma / \mathbb{R}_{+}^{*}$ désigne la projection canonique. On remarquera que pour $q \in C_{0}^{0}\left(\Sigma / \mathbb{R}_{+}^{*} ; \operatorname{End}\left(\mathbb{C}^{N}\right)\right)$, la relation de symétrie $(q \circ \pi)(\rho, x=0, \xi)=(q \circ \pi)(\rho, x=0,-\xi)$ est satisfaite. De plus, comme $\left.\mu\right|_{\mathcal{H}}=0$, la mesure $\hat{\mu}$ détermine uniquement $\mu$. On peut réécrire l'équation (3.9) sur $\tilde{\mu}$ sous forme homogène en posant $\tilde{q}=\left(\lambda^{2} / \tau\right) a$. On obtient

$$
\begin{aligned}
& \forall a \in C_{+}^{\infty, 0}\left(p^{-1}(0), \operatorname{End}\left(\mathbb{C}^{N}\right)\right) \quad\left\langle\tilde{\mu},\left\{\frac{p}{\tau}, a\right\}+\ell^{*} a+a \ell\right\rangle=0 \\
& \text { avec } \ell=\frac{1}{2} \frac{\tau}{\lambda^{2}}\left\{\frac{p}{\tau}, \frac{\lambda^{2}}{\tau}\right\} \mathrm{Id}-i \frac{n}{\tau} .
\end{aligned}
$$

Soit $E_{0}=\mathbb{C}^{N}$ muni de son produit scalaire usuel $\left(e \mid e^{\prime}\right)_{\mathbb{C}^{N}} ;$ soit $s \mapsto \mathcal{L}(s)$ une application $C^{\infty}$ de $\mathbb{R}$ dans $\mathcal{M}_{N}(\mathbb{C})$ et $s \mapsto M(s)$ la solution de l'équation différentielle

$$
\frac{\mathrm{d} M}{\mathrm{~d} s}=\mathcal{L} M, \quad M(s=0)=\mathrm{Id} .
$$

Soit $\mathcal{T}_{0}$ la trivilisation du fibré $\mathbb{R} \times \mathbb{C}^{N}$ sur $\mathbb{R}$

$$
\mathbb{R} \times E_{0} \stackrel{\mathcal{T}_{0}}{\rightarrow} \mathbb{R} \times \mathbb{C}^{N}
$$

$$
(s, e) \longmapsto(s, M(s) e) \text {. }
$$

$\operatorname{Si} b(s) \in \Gamma\left(\mathbb{R}, \operatorname{End}\left(\mathbb{C}^{N}\right)\right.$ est identifiée à la forme hermitienne $\left(b e \mid e^{\prime}\right)_{\mathbb{C}^{N}}$, son image inverse par $\mathcal{T}_{0}$ est

$$
\mathcal{T}_{0}^{*}(b)(s)=M^{*}(s) b(s) M(s) .
$$

Si $\mu$ est une mesure élément du dual de l'espace $C_{0}^{0}\left(\mathbb{R}_{s}, \operatorname{End}\left(\mathbb{C}^{N}\right)\right)$, son image inverse $\mathcal{T}_{0}^{*}(\mu)$ par $\mathcal{T}_{0}$ est alors définie par

$$
\langle\mu, b\rangle=\left\langle\mathcal{T}_{0}^{*}(\mu), M^{*} b M\right\rangle
$$

et on a

LEMME 3.1. - Il y a équivalence entre

i) $\mathcal{T}_{0}^{*}(\mu)$ vérifie l'équation $\frac{\mathrm{d}}{\mathrm{d} s} \mathcal{T}_{0}^{*}(\mu)=0$.

ii) On $a\left\langle\mu, \frac{\mathrm{d} a}{\mathrm{~d} s}+\mathcal{L}^{*} a+a \mathcal{L}\right\rangle=0$ pour tout $a \in C_{0}^{\infty}\left(\mathbb{R}_{s}, \operatorname{End}\left(\mathbb{C}^{N}\right)\right)$.

Démonstration. - Le lemme 3.1 est conséquence de l'identité $\frac{\mathrm{d}}{\mathrm{d} s}\left(M^{*} a M\right)=M^{*}\left(\frac{\mathrm{d} a}{\mathrm{~d} s}+\mathcal{L}^{*} a+\right.$ $a \mathcal{L}) M$.

Compte tenu du lemme 3.1, l'équation (3.11) vérifiée par la mesure $\tilde{\mu}$ est donc formellement équivalente à l'invariance par le flot $\phi(s, \rho)$ de l'image inverse de la mesure $\hat{\mu}$ sur $\Sigma / \mathbb{R}_{+}^{*}$, par la trivialisation $\mathcal{T}$ du fibré $\Sigma / \mathbb{R}_{+}^{*} \times \mathbb{C}^{N}$

$$
\left\{\begin{array}{l}
\left(I \subset \mathbb{R} \times \Sigma /\left.\mathbb{R}_{+}^{*}\right|_{S}\right) \times E_{0} \stackrel{\mathcal{T}}{\longrightarrow} \Sigma / \mathbb{R}_{+}^{*} \times \mathbb{C}^{n} \\
((s, \rho), e) \longmapsto(\phi(s, \rho), M(s, \rho)(e)),
\end{array}\right.
$$

où $S$ est une hypersurface transverse au flot, et où l'application matricielle $s \mapsto M(s, \rho)$ vérifie l'équation différentielle

$$
\frac{\mathrm{d} M}{\mathrm{~d} s}(s, \rho)=\ell(\phi(s, \rho)) M(s, \rho), \quad M(0, \rho)=\mathrm{Id} .
$$


On considérera l'équation (3.17) comme équation différentielle ordinaire en $s$, avec paramètre $\rho \in \Sigma$. On notera qu'à $\rho$ fixé, la fonction $s \mapsto \ell(\phi(s, \rho))$ est bornée, $C^{0}$ sauf en un nombre fini de points $s_{i}$ où elle admet (éventuellement) une discontinuité de première espèce et possède des limites à gauche et à droite en chacun de ces points. Les points de discontinuité $s_{i}$ correspondent aux valeurs de $s$ pour lesquelles $\rho_{i}=\phi\left(s_{i}, \rho\right) \in \Sigma_{1}$, i.e. aux points de réflexion hyperbolique de la trajectoire. Il en résulte l'existence et l'unicité de la solution de l'équation (3.17) pour tout $\rho \in \Sigma$.

La proposition suivante assure la continuité de la trivialisation $\mathcal{T}$ (3.16), ce qui permet de définir sans ambiguïté l'image inverse $\mathcal{T}^{*}(\hat{\mu})$.

Proposition 3.2. - La solution $M(s, \rho)$ de l'équation différentielle (3.17) est continue en $(s, \rho) \in \mathbb{R} \times \Sigma$.

Démonstration. - On a

$$
M(t, \rho)=\mathrm{Id}+\int_{0}^{t} \ell(\phi(s, \rho)) M(s, \rho) \mathrm{d} s,
$$

d'où avec des constantes localement uniformes en $\rho$

$$
\|M(t, \rho)\| \leqslant C ; \quad\left\|M(t, \rho)-M\left(t^{\prime}, \rho\right)\right\| \leqslant C\left|t-t^{\prime}\right| .
$$

Il suffit donc de vérifier pour tout $t$, la continuité en $\rho$ de $M(t, \rho)$. Posons

$$
\begin{aligned}
& \Delta\left(t, \rho_{1}, \rho_{2}\right)=\int_{0}^{t}\left\|\ell\left(\phi\left(s, \rho_{1}\right)\right)-\ell\left(\phi\left(s, \rho_{2}\right)\right)\right\| \mathrm{d} s, \\
& D\left(t, \rho_{1}, \rho_{2}\right)=\left\|M\left(t, \rho_{1}\right)-M\left(t, \rho_{2}\right)\right\| .
\end{aligned}
$$

Les fonctions $\Delta$ et $D$ sont continues en $t$, et on a

$$
D\left(t, \rho_{1}, \rho_{2}\right) \leqslant C\left[\Delta\left(t, \rho_{1}, \rho_{2}\right)+\int_{0}^{t} D\left(s, \rho_{1}, \rho_{2}\right) \mathrm{d} s\right]
$$

Par le lemme de Gronwall, on a donc

$$
D\left(t, \rho_{1}, \rho_{2}\right) \leqslant C \Delta\left(t, \rho_{1}, \rho_{2}\right) \exp (\text { Cte } t) .
$$

La proposition est donc conséquence de

$$
\forall \rho_{2}, \quad \lim _{\rho_{1} \rightarrow \rho_{2}} \Delta\left(t, \rho_{1}, \rho_{2}\right)=0 .
$$

Comme le flot $\phi(s, \rho)$ est continu, et puisque $y, \eta, x, \xi^{2}$ sont des fonctions continues sur $\Sigma$, il suffit de vérifier

$$
\forall \rho_{2}, \quad \lim _{\rho_{1} \rightarrow \rho_{2}} \int_{0}^{t}\left|\xi\left(\phi\left(s, \rho_{1}\right)\right)-\xi\left(\phi\left(s, \rho_{2}\right)\right)\right| \mathrm{d} s=0 .
$$


La fonction $(s, \rho) \mapsto \xi(\phi(s, \rho))$ est uniformément bornée, continue en tout point $\left(s_{0}, \rho_{0}\right)$ tel que $\phi\left(s_{0}, \rho_{0}\right) \notin \Sigma_{1}$, et d'après l'hypothèse $\Sigma^{\infty}=\emptyset$ il n'existe qu'un nombre fini de valeurs de $s \in[0,1]$ telles que $\phi\left(s, \rho_{2}\right) \in \Sigma_{1}$. D'après le théorème de convergence dominée, on obtient (3.25).

\subsection{Le théorème de propagation}

On note $\dot{\sigma}$ l'image de $\sigma \in \Sigma$ dans $\Sigma / \mathbb{R}_{+}^{*}$. Rappelons que $\Sigma / \mathbb{R}_{+}^{*}$ est un espace métrisable. On notera $\operatorname{dist}\left(\dot{\sigma}, \dot{\sigma}^{\prime}\right)$ une distance sur $\Sigma / \mathbb{R}_{+}^{*}$. Soit $\Omega$ un ouvert homogène de $\Sigma$ et $U=\pi^{-1}(\Omega)$. Si $\tilde{\mu}$ est une mesure hermitienne positive élément du dual de $C_{0}^{0}\left(U / \mathbb{R}_{+}^{*}, \operatorname{End}\left(\mathbb{C}^{N}\right)\right)$, et vérifiant $\left.\tilde{\mu}\right|_{\mathcal{H}}=0$ on notera $\hat{\mu}$ l'image de $\tilde{\mu}$ par la projection $\pi: p^{-1}(0) \rightarrow \Sigma$

$$
\langle\hat{\mu}, q\rangle=\langle\tilde{\mu}, q \circ \pi\rangle, \quad q \in C_{0}^{0}\left(\Omega / \mathbb{R}_{+}^{*}, \operatorname{End}(\mathbb{C})\right) .
$$

On dira que $\tilde{\mu}$ vérifie l'équation “ $\dot{\tilde{\mu}}=0$ ” sur $U$ si on a

$$
\left\langle\tilde{\mu},\left\{\frac{p}{\tau}, a\right\}+\ell^{*} a+a \ell\right\rangle=0, \quad \forall a \in C_{+}^{\infty, 0}\left(U, \operatorname{End}\left(\mathbb{C}^{N}\right)\right),
$$

où $\ell(y, \eta, n, \xi) \in C^{\infty, 0}\left(p^{-1}(0), \operatorname{End}\left(\mathbb{C}^{N}\right)\right)$ est défini en (3.11). On note toujours $\mathcal{T}$ la trivialisation du fibré hermitien $\Sigma / \mathbb{R}_{+}^{*} \times \mathbb{C}^{N}$ associée à $\ell$ par (3.16) et (3.17). On dira que $\hat{\mu}$ est invariante sur $\Omega$ si $\mathcal{T}^{*}(\hat{\mu})$ est invariante par le flot de Melrose-Sjöstrand remonté trivialement sur le fibré $\Sigma / \mathbb{R}_{+}^{*} \times \mathbb{C}^{N}$ par la formule

$$
(\rho, e) \longmapsto(\phi(s, \rho), e), \quad \rho \in \Sigma / \mathbb{R}_{+}^{*}, e \in \mathbb{C}^{N}
$$

L'objet de ce paragraphe est de vérifier le théorème de propagation suivant.

THÉORÈME 1. - Les deux propriétés suivantes sont équivalentes.

i) $\hat{\mu}$ est invariante.

ii) $\tilde{\mu}$ vérifie l'équation “ $\dot{\tilde{\mu}}=0$ " et $\left.\tilde{\mu}\right|_{\mathcal{G}^{2,+}}=0$.

Démonstration du théorème 1. - On vérifie d'abord i) $\Rightarrow$ ii).

Soit $a \in C_{+}^{\infty, 0}\left(p^{-1}(0)\right.$, End $\left.\left.\mathbb{C}^{N}\right)\right)$; on a $a(\rho, x=0, \xi)=a(\rho, x=0,-\xi)$, donc $a$ définit une section $\hat{a} \in C_{0}^{0}\left(\Sigma / \mathbb{R}_{+}^{*}, \operatorname{End}\left(\mathbb{C}^{N}\right)\right)$ qui rend commutatif le diagramme

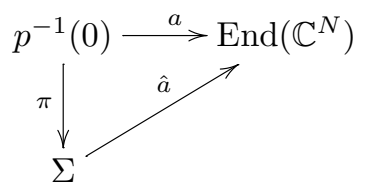

(On identifie $\hat{a}$ à la section homogène de degré zéro de $C_{0}^{0}\left(\Sigma, \operatorname{End}\left(\mathbb{C}^{N}\right)\right)$.) Soit $s \mapsto \phi(s, \rho)$ un rayon. La fonction continue $s \mapsto \hat{a}(\phi(s, \rho))$ est dérivable à gauche et à droite en tout point et on a

$$
\left.\frac{\mathrm{d}}{\mathrm{d} s} \hat{a}(\phi(s, \rho))\right|_{g, d}=\left\{\frac{p}{\tau}, a\right\}\left(\sigma_{g, d}\right)
$$


où $\sigma_{g, d}$ est un des au plus 2 points de $p^{-1}(0)$ éléments de $\pi^{-1}(\phi(s, \rho))$. En particulier, $s \mapsto \hat{a}(\phi(s, \rho))$ est dérivable en tout point $s$ tel que $\phi(s, \rho) \notin \mathcal{H}$ et $y$ vérifie

$$
\frac{\mathrm{d}}{\mathrm{d} s} \hat{a}(\phi(s, \rho))=\left\{\frac{p}{\tau}, a\right\}(\phi(s, \rho)) .
$$

Comme $\left.\hat{\mu}\right|_{\mathcal{H}}=0$ (car $\hat{\mu}$ est invariante et $\mathcal{H}$ est transverse au flot) l'équation (3.27) est conséquence de

$$
\left\langle\hat{\mu}, \frac{\partial \hat{a}}{\partial s}+\ell^{*} \hat{a}+\hat{a} \ell\right\rangle=0, \quad \forall a \in C_{+}^{\infty, 0}\left(p^{-1}(0), \operatorname{End}\left(\mathbb{C}^{N}\right)\right)
$$

Il suffit alors de remarquer que (3.30) résulte comme dans le lemme 3.1 de la construction de la trivialisation $\mathcal{T}$ définie par (3.16) et (3.17). Enfin, on a $\left.\hat{\mu}\right|_{\mathcal{G}^{2,+}}=0$ car $\mathcal{G}^{2,+}$ est transverse au flot de Melrose-Sjöstrand et $\hat{\mu}$ est invariante donc $\left.\tilde{\mu}\right|_{\mathcal{G}^{2,+}}=0$.

Vérifions à présent ii) $\Rightarrow$ i).

Le théorème étant de nature locale près de chaque point $\sigma=(y, \eta, x, \xi)$ de $p^{-1}(0)$, nous procédons par récurrence sur la strate $\Sigma^{j}$ telle que $\pi(\sigma) \in \Sigma^{j}$. Lorsque $\pi(\sigma) \in \Sigma^{0}$ (i.e. dans $x>0$ ), le résultat est conséquence du lemme 3.1 , et près des points de réflexion hyperbolique, $\pi(\sigma) \in \Sigma^{1}$, du lemme 3.1 et de la formule des sauts (on pourrait aussi utiliser le calcul explicite de la paramétrixe près des points hyperboliques, voir [4] ou [6] pour le cas $N=1$ ).

Pour traiter les cas $\pi(\sigma) \in \Sigma^{j}$, avec $j \geqslant 2$, on commence (voir la proposition 3.3) par construire des mesures auxiliaires qui permettent de généraliser l'équation (3.27) à des fonctions $b \in C^{\infty, 0}\left(p^{-1}(0), \operatorname{End}\left(\mathbb{C}^{N}\right)\right), C^{\infty}$ à support compact, homogènes de degré zéro de $p^{-1}(0)$ dans $\operatorname{End}\left(\mathbb{C}^{N}\right)$, mais ne vérifiant pas nécessairement la relation de symétrie entre les points $\xi$ et $-\xi$ $\operatorname{sur} x=0$.

Tout élément $b$ de $C^{\infty, 0}\left(p^{-1}(0), \operatorname{End}\left(\mathbb{C}^{N}\right)\right)$ se décompose d'une infinité de façons sous la forme

$$
b=a^{0}+\xi a^{-1}, \quad a^{j} \in C_{+}^{\infty, j}\left(p^{-1}(0), \operatorname{End}\left(\mathbb{C}^{N}\right)\right),
$$

où les $a^{j}$ sont des fonctions paires de $\xi$ sur $x=0$. On peut par exemple prendre

$$
a^{0}(\rho, x, \xi)=(b(\rho, x, \xi)+b(\rho, x,-\xi)) / 2
$$

et

$$
a^{-1}(\rho, x, \xi)=(b(\rho, x, \xi)-b(\rho, x,-\xi)) / 2 \xi
$$

On a

$$
\left\langle\tilde{\mu},\left\{\frac{p}{\tau}, b\right\}+\ell^{*} b+b \ell\right\rangle=\left\langle\tilde{\mu},\left\{\frac{p}{\tau}, \xi a^{-1}\right\}+\xi \ell^{*} a^{-1}+\xi a^{-1} \ell\right\rangle \stackrel{\text { def }}{=} I .
$$

On choisit $\chi \in C^{\infty}(\mathbb{R})$ vérifiant $\chi(x)=1$ pour $x \leqslant 1, \chi(x)=0$ pour $x \geqslant 2$ et $\chi^{\prime} \leqslant 0$; on pose $\phi=1-\chi$. Pour tout $\varepsilon>0$ petit on a $\xi a^{-1} \phi\left(\frac{x}{\varepsilon}\right) \in C_{+}^{\infty, 0}\left(p^{-1}(0), \operatorname{End}\left(\mathbb{C}^{N}\right)\right)$ d'où

$$
I=\left\langle\tilde{\mu},\left\{\frac{p}{\tau}, \xi a^{-1} \chi\left(\frac{x}{\varepsilon}\right)\right\}\right\rangle+\left\langle\tilde{\mu}, \xi \chi\left(\frac{x}{\varepsilon}\right)\left(\ell^{*} a^{-1}+a^{-1} \ell\right)\right\rangle .
$$


Le terme $\left\langle\tilde{\mu}, \xi \chi\left(\frac{x}{\varepsilon}\right)\left(\ell^{*} a^{-1}+a^{-1} \ell\right)\right\rangle$ converge quand $\varepsilon \rightarrow 0$ par le théorème de convergence dominée vers $\left\langle\tilde{\mu} 1_{x=0} \xi, \ell^{*} a^{-1}+a^{-1} \ell\right\rangle$ qui est nul car $\left.\tilde{\mu}\right|_{\mathcal{H}}=0$ et $\left.\xi\right|_{\Sigma^{j}}=0$ pour $j \geqslant 2$. On a donc

$$
\left\{\begin{array}{l}
I=I_{1}^{\varepsilon}+I_{2}^{\varepsilon}+I_{3}^{\varepsilon}, \\
I_{1}^{\varepsilon}=\left\langle\tilde{\mu}, \xi \chi\left(\frac{x}{\varepsilon}\right)\left\{\frac{p}{\tau}, a^{-1}\right\}\right\rangle, \\
I_{2}^{\varepsilon}=\left\langle\tilde{\mu}, \frac{1}{\tau}\left(-\frac{\partial p}{\partial x}\right) a^{-1} \chi\left(\frac{x}{\varepsilon}\right)\right\rangle, \\
I_{3}^{\varepsilon}=\left\langle\tilde{\mu}, \xi a^{-1}\left\{\frac{p}{\tau}, \chi\left(\frac{x}{\varepsilon}\right)\right\}\right\rangle .
\end{array}\right.
$$

Comme précédemment, on a $\lim _{\varepsilon \rightarrow 0} I_{1}^{\varepsilon}=0$. Par convergence dominée, on obtient

$$
\lim _{\varepsilon \rightarrow 0} I_{2}^{\varepsilon}=-\left\langle\tilde{\mu} 1_{x=0}, a^{-1} \frac{r_{1}}{\tau}\right\rangle .
$$

Pour calculer la limite du terme $I_{3}^{\varepsilon}$, on utilise

$$
\xi a^{-1} \phi\left(\frac{x}{\varepsilon}\right) \in C_{+}^{\infty, 0}\left(p^{-1}(0), \operatorname{End}\left(\mathbb{C}^{N}\right)\right)
$$

d'où

$$
\left\langle\tilde{\mu},\left\{\frac{p}{\tau}, \xi a^{-1} \phi\left(\frac{x}{\varepsilon}\right)\right\}+\xi \phi\left(\frac{x}{\varepsilon}\right)\left(\ell^{*} a^{-1}+a^{-1} \ell\right)\right\rangle=0 .
$$

On a

$$
\left\langle\tilde{\mu},\left\{\frac{p}{\tau}, \xi a^{-1} \phi\left(\frac{x}{\varepsilon}\right)\right\}\right\rangle=\left\langle\tilde{\mu},\left\{\frac{p}{\tau}, \xi a^{-1}\right\} \phi\left(\frac{x}{\varepsilon}\right)\right\rangle-I_{3}^{\varepsilon} .
$$

Car d'après (3.36)

$$
I_{3}^{\varepsilon}=2\left\langle\tilde{\mu}, \frac{\xi^{2}}{\tau \varepsilon} a^{-1} \chi^{\prime}\left(\frac{x}{\varepsilon}\right)\right\rangle
$$

d'où

$$
I_{3}^{\varepsilon}=\left\langle\tilde{\mu},\left(\left\{\frac{p}{\tau}, \xi a^{-1}\right\}+\left(\xi \ell^{*} a^{-1}+\xi a^{-1} \ell\right)\right) \phi\left(\frac{x}{\varepsilon}\right)\right\rangle .
$$

Lemme 3.2. - On a pour tout $a^{-1}, \lim _{\varepsilon \rightarrow 0} I_{3}^{\varepsilon}\left(a^{-1}\right)=\left\langle T_{0}, \tau a^{-1}\right\rangle$ où $T_{0}$ est une mesure hermitienne positive, élément du dual de $C_{0}^{0}\left(\Sigma / \mathbb{R}_{+}^{*}, \operatorname{End}\left(\mathbb{C}^{N}\right)\right)$, et portée par $\mathcal{H}=\Sigma_{1}$.

Démonstration. - On a d'après (3.42)

$$
\lim _{\varepsilon \rightarrow 0} I_{3}^{\varepsilon}\left(a^{-1}\right)=\left\langle\tilde{\mu} 1_{x>0},\left\{\frac{p}{\tau}, \xi a^{-1}\right\}+\xi \ell^{*} a^{-1}+\xi a^{-1} \ell\right\rangle
$$

et pour $a^{-1}$ hermitienne positive, on a $I_{3}^{\varepsilon}\left(a^{-1}\right) \geqslant 0$, d'après (3.41). Comme une limite faible de mesures hermitiennes positives est une mesure hermitienne positive, on obtient l'existence de $T_{0}$. La mesure $T_{0}$ est portée par $\{x=0\}$ d'après (3.41). Comme

$$
\tau a^{-1} \mapsto\left\langle\tilde{\mu} 1_{x>0}, a^{-1}\left\{\frac{p}{\tau}, \xi\right\}+\xi \ell^{*} a^{-1}+\xi a^{-1} \ell\right\rangle
$$


est une mesure qui ne charge pas $x=0$, on en déduit que

$$
a^{-1} \longmapsto\left\langle\tilde{\mu} 1_{x>0},\left\{\frac{p}{\tau}, a^{-1}\right\} \xi\right\rangle \stackrel{\text { def }}{=}\left\langle S, a^{-1} \tau\right\rangle
$$

est une mesure, et il reste à vérifier que $S$ ne charge pas $\bigcup_{j \geqslant 2} \Sigma^{j}=\{x=\xi=0\}$; on remplace alors $a^{-1}$ par $a^{-1} \theta\left(\frac{x}{\beta}\right) \psi\left(\frac{\xi}{\alpha \tau}\right)$ avec $\theta, \psi \in C_{0}^{\infty}(\mathbb{R})$, paire, $\alpha \rightarrow 0, \beta \rightarrow 0$ et $\frac{\alpha^{2}}{\beta} \rightarrow 0$. On a

$$
\begin{aligned}
& \left\langle S, \tau a^{-1} \theta\left(\frac{x}{\beta}\right) \psi\left(\frac{\xi}{\alpha \tau}\right)\right\rangle=S_{1}+S_{2}+S_{3}+S_{4}, \\
& S_{1}=\left\langle\tilde{\mu} 1_{x>0},\left\{\frac{p}{\tau}, a^{-1}\right\} \theta\left(\frac{x}{\beta}\right) \psi\left(\frac{\xi}{\alpha \tau}\right) \xi\right\rangle \longrightarrow 0, \\
& S_{2}=\left\langle\tilde{\mu} 1_{x>0},-\frac{2 \xi^{2}}{\beta \tau} \theta^{\prime}\left(\frac{x}{\beta}\right) \psi\left(\frac{\xi}{\alpha \tau}\right) a^{-1}\right\rangle \longrightarrow 0,
\end{aligned}
$$

par convergence dominée car

$$
\begin{gathered}
\left|\frac{\xi^{2}}{\beta \tau} \psi\left(\frac{\xi}{\alpha \tau}\right) \theta^{\prime}\left(\frac{x}{\beta}\right) a^{-1}\right| \leqslant \text { Cte } \frac{\alpha^{2}}{\beta} \longrightarrow 0 . \\
S_{3}=\left\langle\tilde{\mu} 1_{x>0},-\frac{\xi}{\tau^{2} \alpha} \psi^{\prime}\left(\frac{\xi}{\alpha \tau}\right) \frac{\partial r}{\partial x} \theta\left(\frac{x}{\beta}\right) a^{-1}\right\rangle \longrightarrow 0,
\end{gathered}
$$

car $\frac{a^{-1} \xi}{\tau^{2} \alpha} \psi^{\prime}\left(\frac{\xi}{\alpha \tau}\right) \frac{\partial r}{\partial x} \theta\left(\frac{x}{\beta}\right) 1_{x>0}$ tend vers zéro partout et est borné et de même

$$
S_{4}=\left\langle\tilde{\mu} 1_{x>0}, \frac{\xi^{2}}{\alpha} \psi^{\prime}\left(\frac{\xi}{\alpha \tau}\right)\left\{\frac{p}{\tau}, \frac{1}{\tau}\right\} \theta\left(\frac{x}{\beta}\right) a^{-1}\right\rangle \longrightarrow 0,
$$

ce qui achève la preuve du lemme.

D'après (3.36) à (3.41) et le lemme 3.2, on a obtenu la

Proposition 3.3. - Pour tout $a^{j} \in C_{+}^{\infty, j}\left(p^{-1}(0), \operatorname{End}\left(\mathbb{C}^{N}\right)\right)$ on a avec $b=a^{0}+\xi a^{-1}$

$$
\left\langle\tilde{\mu},\left\{\frac{p}{\tau}, b\right\}+\ell^{*} b+b \ell\right\rangle=\left\langle-\frac{r_{1}}{\tau^{2}} \mathbf{1}_{x=0} \tilde{\mu}+T_{0}(\tilde{\mu}), \tau a^{-1}\right\rangle .
$$

La mesure $\frac{r_{1}}{\tau^{2}} \mathbf{1}_{x=0} \tilde{\mu}$ est portée par $\bigcup_{j \geqslant 2} \Sigma_{j}$. La mesure hermitienne positive $T_{0}(\tilde{\mu})$, portée par $\Sigma_{1}=\mathcal{H}$ étant définie par

$$
\left\langle T_{0}(\tilde{\mu}), \tau a^{-1}\right\rangle=\lim _{\varepsilon \rightarrow 0} 2\left\langle\tilde{\mu}, \frac{\xi^{2}}{\tau \varepsilon} a^{-1} \chi^{\prime}\left(\frac{x}{\varepsilon}\right)\right\rangle,
$$

où $\chi \in C^{\infty}(\mathbb{R})$ est croissante, vérifie $\chi(x)=0$ pour $x \leqslant 1$ et $\phi(x)=1$ pour $x \geqslant 2$.

Soit à présent $j_{0} \geqslant 2$ un entier, $\sigma_{0}$ un point de $\Sigma^{j_{0}}$. Nous allons vérifier l'assertion i) $\Rightarrow$ ii) du théorème $1 \mathrm{au}$ voisinage de $\sigma^{0}$ par récurrence sur $j_{0}$ en supposant qu'elle est vraie au 
voisinage de tout point de $\Sigma^{j}$ pour $j<j_{0}$. On notera $(z, \zeta)$ un système de coordonnées symplectiques homogènes près de $\sigma_{0}$ tel que $z\left(\sigma_{0}\right)=0$ et $r_{0}=\zeta_{1} \tau$ près de $\sigma_{0}$ (ce système existe d'après le théorème de la base symplectique incomplète de Darboux). On a donc $\zeta_{1}\left(\sigma_{0}\right)=0$ et $p=-\xi^{2}+r=-\xi^{2}+\zeta_{1} \tau+x r_{1}(z, \zeta)+\mathcal{O}\left(x^{2}\right)$. Les fonctions $z, \zeta$ sont dérivables sur les rayons et vérifient

$$
\frac{\mathrm{d} z}{\mathrm{~d} s}=\frac{\partial r}{\partial \zeta}(x(s), z(s), \zeta(s)), \quad \frac{\mathrm{d} \zeta}{\mathrm{d} s}=-\frac{\partial r}{\partial z}(x(s), z(s), \zeta(s)) .
$$

En particulier, près de $\sigma_{0}$, on a $\frac{\mathrm{d} z_{1}}{\mathrm{~d} s} \sim \tau>0$, donc $s \mapsto z_{1}(s)$ est strictement croissant sur les rayons et l'hypersurface $S=\left\{z_{1}=0\right\}$ est transverse au flot. Soit $\omega$ un petit voisinage ouvert homogène de $\sigma_{0}$ dans $S$ et $\Omega$ l'ouvert de $\Sigma$,

$$
\Omega_{\varepsilon_{0}}=\left\{\phi(s, \sigma) ; \sigma \in \omega,|s|<\varepsilon_{0}\right\} .
$$

Quitte à diminuer $\omega$, on peut supposer que $\Omega_{\varepsilon_{0}} \cap \Sigma_{j}=\emptyset, \forall j>j_{0}$. Soit $\omega_{j_{0}}=\{\sigma \in \omega, \exists s$, $\left.|s|<\varepsilon_{0}, \phi(s, \sigma) \in \Sigma^{j_{0}}\right\}$ et soit $F$ l'adhérence dans $\Omega_{\varepsilon_{0}}$ de $\left\{\phi(s, \sigma) ;|s|<\varepsilon_{0}, \sigma \in \omega_{j_{0}}\right\}$, et $V$ l'ouvert de $\Omega_{\varepsilon_{0}}, V=\Omega_{\varepsilon_{0}} \backslash F$. Il existe alors un ouvert $\omega_{v}$ de $\omega$ tel que qu'on ait

$$
V=\left\{\phi(s, \sigma), \sigma \in \omega_{v},|s|<\varepsilon_{0}\right\} .
$$

Comme $\bigcup_{j>j_{0}} \Sigma^{j}$ est fermé, en choisissant $\omega$ et $\varepsilon_{0}$ assez petits, on a $V \cap \Sigma^{j}=\phi$ pour tout $j \geqslant j_{0}$. Comme on a “ $\dot{\tilde{\mu}}=0$ " sur l'ouvert $V$, et $\left.\tilde{\mu}\right|_{\mathcal{G}} ^{2,+}=0,\left.\hat{\mu}\right|_{V}$ est invariante par hypothèse de récurrence et $V$ étant un tube de rayon de la forme (3.55), il en résulte que la mesure hermitienne positive $\left.\mathbf{1}_{V / \mathbb{R}_{+}^{*}} \hat{\mu}\right|_{\left(\Omega_{\varepsilon_{0}} / \mathbb{R}_{+}^{*}\right)}$ est invariante sur $\Omega_{\varepsilon_{0}}$. Comme on a déjà vérifié l'assertion i) $\Rightarrow$ ii) du théorème 1 , on a donc " $\mathbf{1}_{V / \mathbb{R}_{+}^{*}} \tilde{\mu}=0$ " sur $\Omega_{\varepsilon_{0}}$. D' après l'identité $\left.\tilde{\mu}\right|_{\Omega_{\varepsilon_{0}}}=\left.\mathbf{1}_{V} \tilde{\mu}\right|_{\Omega_{\varepsilon_{0}}}+\left.\mathbf{1}_{F} \tilde{\mu}\right|_{\Omega_{\varepsilon_{0}}}$, on en déduit " $\left.\mathbf{1}_{F} \dot{\tilde{\mu}}\right|_{\Omega_{\varepsilon_{0}}}=0$ " sur $\Omega_{\varepsilon_{0}}$. Comme on a pour $\omega$ et $\varepsilon_{0}$ assez petits

$$
\left\{\begin{array}{l}
F=\left\{\phi(s, \sigma),|s|<\varepsilon_{0}, \sigma \in \tilde{\omega}\right\} \quad \text { avec } \\
\tilde{\omega}=\left\{\sigma \in \omega, \exists s,|s| \leqslant \varepsilon_{0}, \phi(s, \sigma) \in \Sigma^{j_{0}}\right\}
\end{array}\right.
$$

on peut donc supposer que près de $\sigma_{0}$, la mesure $\tilde{\mu}$ est portée par une réunion de rayons qui rencontrent $\Sigma^{j_{0}}$. On va à présent distinguer trois cas : $\sigma_{0} \in \mathcal{G}^{2,+}, \sigma_{0} \in \mathcal{G}^{2,-}, \sigma_{0} \in \Sigma^{j_{0}}, j_{0} \geqslant 3$.

\subsubsection{1er cas}

$\sigma_{0} \in \mathcal{G}^{2,+}$. C'est le cas strictement diffractif. D'après l'hypothèse, on peut supposer $\tilde{\mu}$ portée par les rayons qui rencontrent $\mathcal{G}^{2,+}$, près de $\sigma_{0}$. D'après (3.51), $T_{0}(\tilde{\mu}) 1_{\mathcal{H}} \equiv 0$ et puisque $T_{0}(\tilde{\mu})$ est portée par $\mathcal{H}$ d'après la proposition 3.3 , on a $T_{0}(\tilde{\mu}) \equiv 0$ près de $\sigma_{0}$, et comme par hypothèse on a $\left.\tilde{\mu}\right|_{\mathcal{G}^{2,+}}=0$ on déduit de (3.51)

$$
\left\langle\tilde{\mu},\left\{\frac{p}{\tau}, b\right\}+\ell^{*} b+b \ell\right\rangle=0, \quad \forall b \in C^{\infty, 0}\left(p^{-1}(0), \operatorname{End}\left(\mathbb{C}^{N}\right)\right) .
$$

Comme les rayons issus de $\mathcal{G}^{2,+}$ sont les courbes intégrales de $H_{p / \tau}$ contenues dans $p=0$ et restent dans $x \geqslant 0$, la mesure $\hat{\mu}$ est invariante d'après le lemme 3.1.

\subsubsection{2ème cas}

$\sigma_{0} \in \mathcal{G}^{2,-}$. C'est le cas strictement glissant. D'après l'hypothèse, on peut supposer $\tilde{\mu}$ portée par les rayons qui rencontrent $\mathcal{G}^{2,-}$ près de $\sigma_{0}$. Or ces rayons sont tout entiers contenus dans 
$\mathcal{G}^{2,-}$ près de $\sigma_{0}$, de sorte qu'on peut supposer $\tilde{\mu}$ à support dans $\mathcal{G}^{2,-}$. On a

$$
\forall a \in C_{+}^{\infty, 0}\left(p^{-1}(0), \operatorname{End}\left(\mathbb{C}^{N}\right)\right) \quad\left\langle\tilde{\mu},\left\{\frac{p}{\tau}, a\right\}+\ell^{*} a+a \ell\right\rangle=0
$$

et

$$
\left.\left\{\frac{p}{\tau}, a\right\}\right|_{\mathcal{G}^{2,-}}=\left.\frac{1}{\tau} H_{r_{0}}\left(\left.a\right|_{x=0}\right)\right|_{r_{0}=0} \quad \text { pour } a \in C_{+}^{\infty, 0}\left(p^{-1}(0), \operatorname{End}\left(\mathbb{C}^{N}\right)\right)
$$

(car $\left.a\right|_{x=0}$ est paire en $\xi$ donc $\left.\frac{\partial}{\partial \xi} a\right|_{x=0, \xi=0}=0$ ). De plus, l'application de $C_{+}^{\infty, 0}\left(p^{-1}(0)\right.$, $\left.\operatorname{End}\left(\mathbb{C}^{N}\right)\right)$ dans $C^{\infty, 0}\left(\mathcal{G}^{2,-}, \operatorname{End}\left(\mathbb{C}^{N}\right)\right),\left.a \mapsto a\right|_{x=0, \xi=0}$ est surjective. L'assertion i) $\Rightarrow$ ii) du théorème 1 est donc d'après (3.58) conséquence du lemme 3.1

\subsubsection{3ème cas}

$\sigma_{0} \in \Sigma^{j_{0}}, j_{0} \geqslant 3$. Posons $j_{0}=k+3$, on a

$$
\Sigma^{j_{0}}=\left\{(z, \zeta) ; r_{0}(z, \zeta)=\tau \zeta_{1}=0 ; \partial_{z_{1}}^{\ell} r_{1}(z, \zeta)=0, \forall \ell \leqslant k, \partial_{z_{1}}^{k+1} r_{1}(z, \zeta) \neq 0\right\}
$$

Soit $g_{k}(z, \zeta)=\partial_{z_{1}}^{k} r_{1}(z, \zeta)$; on a donc

$$
\Sigma^{j_{0}} \subset\left\{(z, \zeta) ; g_{k}(z, \zeta)=0, \partial_{z_{1}} g_{k} \neq 0\right\} \stackrel{\text { def }}{=} G .
$$

Par le théorème des fonctions implicites, $G$ est localement près de $\sigma_{0}$ une hypersurface lisse de la forme $G=\left\{z_{1}=\Theta\left(z^{\prime}, \zeta\right)\right\}$ avec $z=\left(z_{1}, z^{\prime}\right)$. Notons à nouveau $F$ la réunion des rayons près de $\sigma_{0}$ qui rencontrent $\Sigma^{j 0}$. Sur chaque rayon, $z_{1}$ est une fonction strictement croissante de $s$ et le flot est transverse à $G$ d'après (3.53). On pose

$$
\left\{\begin{array}{l}
F^{+}=\left\{(z, \zeta, x, \xi) \in F, z_{1}>\Theta\left(z^{\prime}, \zeta\right)\right\}, \\
F^{-}=\left\{(z, \zeta, x, \xi) \in F, z_{1}<\Theta\left(z^{\prime}, \zeta\right)\right\}, \\
F^{0}=\left\{(z, \zeta, x, \xi) \in F, z_{1}=\Theta\left(z^{\prime}, \zeta\right)\right\} \supset \Sigma^{j_{0}} \cap F .
\end{array}\right.
$$

On identifie $G$ à son image dans $\Sigma$. On peut supposer $\tilde{\mu}$ à support dans $F=F^{-} \cup F^{0} \cup F^{+}$. On pose $\tilde{\mu}^{ \pm}=\left.\tilde{\mu}\right|_{ \pm z_{1}>\Theta\left(\zeta^{\prime}, \zeta\right)}$. Par hypothèse de récurrence $\left(F^{ \pm} \cap \Sigma^{j}=\phi\right.$ pour $\left.j \geqslant j_{0}\right)$ les mesures $\hat{\mu}^{ \pm}$sont invariantes sur les ouverts $\pm z_{1}>\Theta\left(z^{\prime}, \zeta\right)$. On peut les prolonger par transport en mesures invariantes à support dans $F$, qu'on notera $\tilde{\mu}^{ \pm}$. On a donc près de $\sigma_{0}$

$$
\tilde{\mu}=\mathbf{1}_{z_{1}>\Theta} \tilde{\mu}^{+}+\mu_{0}+\mathbf{1}_{z_{1}<\Theta} \tilde{\mu}^{-},
$$

où la mesure $\mu_{0}$ est portée par $F^{0}$. L'équation de propagation (3.27) implique

$$
\left\langle\mu_{0},\left\{\frac{p}{\tau}, a\right\}+\ell^{*} a+a \ell\right\rangle=-\left\langle\mathbf{1}_{z_{1}>\Theta} \mu^{+}+\mathbf{1}_{z_{1}<\Theta} \mu^{-},\left\{\frac{p}{\tau}, a\right\}+\ell^{*} a+a \ell\right\rangle
$$

pour tout $a \in C_{+}^{\infty, 0}\left(U, \operatorname{End}\left(\mathbb{C}^{N}\right)\right)$ à support près de $\sigma_{0}$. Notons $\operatorname{Tr}\left(\mu^{ \pm}\right)$les mesures à support dans $F_{0}$, qui sont définies comme les traces sur $F_{0}$ des mesures $\tilde{\mu}^{ \pm}$. On a alors

$$
\left\langle\mu_{0},\left\{\frac{p}{\tau}, a\right\}+\ell^{*} a+a \ell\right\rangle=-\left\langle\operatorname{tr}\left(\mu^{+}\right)-\operatorname{tr}\left(\mu^{-}\right), a\right\rangle .
$$


Il en résulte

$$
\left|\left\langle\mu_{0},\left\{\frac{p}{\tau}, a\right\}\right\rangle\right| \leqslant \text { Cte }\|a\|_{L^{\infty}} .
$$

Or on a $\left.\left\{\frac{p}{\tau}, a\right\}\right|_{F_{0}}=\left.\left\{\frac{r_{0}}{\tau}, a\right\}\right|_{F_{0}}=\left.\frac{\partial a}{\partial z_{1}}\right|_{F_{0}}$. L'inégalité (3.66) implique donc que $\partial_{z_{1}} \mu_{0}$ est une mesure, mais comme $\mu_{0}$ est portée par $F^{0}$ qui est transverse à $\partial_{z_{1}}$, on en déduit $\mu_{0} \equiv 0$, et l'égalité (3.65) entraîne alors $\operatorname{tr}\left(\mu^{+}\right)=\operatorname{tr}\left(\mu^{-}\right)$. D'après l'identité (3.63), on a donc $\tilde{\mu}=\mu^{+}=$ $\mu^{-}$ce qui prouve que $\hat{\mu}$ est invariante.

\subsection{La condition $\left.\tilde{\mu}\right|_{\mathcal{G}^{2,+}}=0$}

Soit $\left(u^{k}\right)_{k \in \mathbb{N}}$ une suite bornée de $H^{1}$ convergeant faiblement vers zéro, vérifiant l'équation $P u^{k}=v^{k}$ (où $v^{k}$ vérifie la relation (2.21) dans $x>0$ ), et la condition aux limites (2.80) sur $x=0$. Soient $\mu, \tilde{\mu}, \hat{\mu}$ les mesures associées à la suite $\left(u^{k}\right)$. D'après (3.11), on a “ “ $\dot{\tilde{\mu}}=0$ ”, et d'après le théorème 1,1 'invariance de la mesure $\hat{\mu}$ va être conséquence de la propriété $\left.\tilde{\mu}\right|_{\mathcal{G}^{2,+}}=0$.

LEMME 3.4. - On a $\left.\tilde{\mu}\right|_{\mathcal{G}^{2,+}}=0$ si et seulement si pour tout $\rho \in \mathcal{G}^{2,+}$ tout $a^{j} \in C_{+}^{\infty, j}\left(p^{-1}(0)\right.$, $\left.\operatorname{End}\left(\mathbb{C}^{N}\right)\right)$ à support près de $\rho$ avec $a^{-1}$ hermitienne positive, et en posant $b=a^{0}+\xi a^{-1}$, la mesure $\tilde{\mu}$ vérifie l'inégalité

$$
\left\langle\tilde{\mu},\left\{\frac{p}{\tau}, b\right\}+\ell^{*} b+b \ell\right\rangle \geqslant 0 .
$$

Démonstration. - En effet, $\mathcal{G}^{2,+}$ est défini par l'inéquation $r_{1}>0$. Le lemme 3.4 est donc conséquence de la formule (3.51) de la proposition 3.3, puisque la mesure positive $T_{0}(\tilde{\mu})$, portée $\operatorname{par} \Sigma_{1}=\mathcal{H}$, ne charge pas $\mathcal{G}^{2,+}$.

On remarquera que (3.67) n'est pas une conséquence de l'équation “ $\dot{\tilde{\mu}}=0$ ” puisque l'on teste ici contre une classe plus grande de fonctions.

Soit alors $q \in C_{0}^{\infty}\left(p^{-1}(0), \operatorname{End}\left(\mathbb{C}^{N}\right)\right)$, homogène de degré 1 , à support près de $(x=0$, $\left.\xi=0, \rho_{0}\right)$. En écrivant $q=\left.\left(q_{0} \xi+q_{1}\right)\right|_{p^{-1}(0)}$ comme dans (2.99), on a d'après (2.100) et (2.101) et le lemme 2.9, avec $\lambda^{2}=|\eta|^{2}\left(1+\theta^{2}\right), \theta=\frac{\xi}{|\eta|}$

$$
\left\langle\mu, \frac{\{p, q\}+i\left(m^{*} q-q m\right)}{\lambda^{2}}\right\rangle=\left\langle\nu, q_{0}+b^{*} q_{0} r b+q_{1} b+b^{*} q_{1}\right\rangle .
$$

Comme $\nu$ est une mesure hermitienne positive (voir (2.87)), la relation (3.67) est conséquence, d'après (3.8), de l'implication : si $a^{0}, a^{-1}, q_{0}, q_{1}$ sont liés sur $p^{-1}(0)$ par la relation

$$
J_{\xi}^{* 1 / 2}\left(q_{0} \xi+q_{1}\right) J_{\xi}^{1 / 2}=\lambda\left(a^{0}+\xi a^{-1}\right)
$$

alors $a^{-1} \geqslant 0$ implique $\left.\left(q_{0}+b^{*} q_{0} r b+q_{1} b+b^{*} q_{1}\right)\right|_{x=0} \geqslant 0$. Comme $\lambda$ est une fonction paire de $\xi$, il s'agit donc de calculer la partie impaire en $\xi$ de la fonction matricielle sur $p^{-1}(0) \cap\{x=0\}$

$$
\xi \longmapsto J_{\xi}^{* 1 / 2}\left(q_{0} \xi+q_{1}\right) J_{\xi}^{1 / 2}
$$

Soit donc

$$
M=\frac{1}{\xi}\left(J_{\xi}^{* 1 / 2}\left(q_{0} \xi+q_{1}\right) J_{\xi}^{1 / 2}-J_{-\xi}^{* 1 / 2}\left(-q_{0} \xi+q_{1}\right) J_{-\xi}^{1 / 2}\right) .
$$


On doit vérifier $M \geqslant 0 \Rightarrow q_{0}+b^{*} q_{0} r b+q_{1} b+b^{*} q_{1} \geqslant 0$ sur $\{x=0\} \cap p^{-1}(0)$. On a $M \geqslant 0 \Leftrightarrow$ $N \geqslant 0$ avec

$$
N=\frac{1}{\xi}\left(J_{\xi}^{*}\left(q_{0} \xi+q_{1}\right) J_{\xi}+\left(q_{0} \xi-q_{1}\right)\right)
$$

et d'après (2.93), $N \geqslant 0$ équivaut à $L \geqslant 0$ avec

$$
L=\frac{1}{\xi}\left(L_{\xi}-L_{-\xi}\right) ; \quad L_{\xi}=\left(\mathrm{Id}-\xi b^{*}\right)^{-1}\left(q_{0} \xi+q_{1}\right)(\mathrm{Id}-\xi b)^{-1} .
$$

Soit $S_{\xi}=(\operatorname{Id}-\xi b)(\operatorname{Id}+\xi b)$; on a $S_{\xi}=S_{-\xi}$ et $L \geqslant 0$ équivaut à $\tilde{L} \geqslant 0$ avec $\tilde{L}=S_{\xi}^{*} L S_{\xi}$. On a

$$
\tilde{L}=\frac{1}{\xi}\left(\tilde{L}_{\xi}-\tilde{L}_{-\xi}\right) ; \quad \tilde{L}_{\xi}=S_{\xi}^{*} L_{\xi} S_{\xi}
$$

et $\operatorname{sur} p^{-1}(0)=\left\{\xi^{2}=r\right\}$

$$
\left\{\begin{aligned}
\tilde{L}_{\xi} & =\left(1+\xi b^{*}\right)\left(q_{0} \xi+q_{1}\right)(1+\xi b) \\
& =\left[q_{1}+r\left(b^{*} q_{0}+q_{0} b+b^{*} q_{1} b\right)\right]+\xi\left[q_{0}+b^{*} q_{0} r b+q_{1} b+b^{*} q_{1}\right],
\end{aligned}\right.
$$

d'où sur $p^{-1}(0) \cap\{x=0\}$,

$$
\tilde{L} \geqslant 0 \Longleftrightarrow q_{0}+b^{*} q_{0} r_{0} b+q_{1} b+b^{*} q_{1} \geqslant 0,
$$

ce qui achève la vérification de l'invariance de la mesure $\hat{\mu}$.

\section{Le système de Lamé, notations}

\subsection{Ondes transversale et longitudinale}

On considère le système de Lamé dans un domaine régulier $\left(C^{\infty}\right), \Omega \subset \mathbb{R}^{3}$ et borné :

$$
\left\{\begin{array}{l}
\left(\partial_{t}^{2}-\mu \Delta-(\lambda+\mu) \nabla \operatorname{div}\right) u=\left(\partial_{t}^{2}+A\right) u=0, \\
\left.u\right|_{\partial \Omega}=0, \\
\left.u\right|_{t=0}=\left.u_{0} \in H_{0}^{1}(\Omega)^{3} \quad \partial_{t} u\right|_{t=0}=u_{1} \in L^{2}(\Omega)^{3},
\end{array}\right.
$$

où $\lambda, \mu$ sont les coefficients de Lamé qui vérifient $\mu>0$ et $\lambda+2 \mu>0$. On supposera $\mu \neq \lambda+2 \mu$. On a une énergie naturelle qui est un invariant de l'évolution :

$$
E(u)(t)=\frac{1}{2} \int_{\Omega}\left|\partial_{t} u\right|^{2}+\mu|\nabla u|^{2}+(\lambda+\mu)|\operatorname{div} u|^{2} .
$$

On peut alors décomposer la solution $u$ en somme de deux termes $u_{L}$ et $u_{T}$ de rotationnel et de divergence nuls respectivement :

LEMME 4.1. - Il existe $(\varphi, \psi) \in H_{\mathrm{loc}}^{2}\left(\mathbb{R}_{t} \times \bar{\Omega}\right)^{1+3}$ vérifiant

1. $u=\nabla \varphi+\operatorname{rot} \psi=u_{L}+u_{T}, u_{L}=\nabla \varphi, u_{T}=\operatorname{rot} \psi$,

2. $\left(\partial_{t}^{2}-c_{L}^{2} \Delta\right) \varphi=0,\left(\partial_{t}^{2}-c_{T}^{2} \Delta\right) \psi=0$,

3. $\operatorname{div} \psi=0, \operatorname{rot} u_{L}=0, \operatorname{div} u_{T}=0$. 
4. Il existe $C>0$ tel que pour tout intervalle $I \Subset \mathbb{R}$

$$
\begin{aligned}
& \|\varphi\|_{H^{2}(I \times \Omega)}^{2} \leqslant C|I| E(u), \\
& \|\psi\|_{H^{2}(I \times \Omega)}^{2} \leqslant C|I| E(u),
\end{aligned}
$$

en particulier si on note

$$
E_{L, T}\left(u_{L, T}\right)=\frac{1}{2} \int_{\Omega}\left|\partial_{t} u_{L, T}\right|^{2}+c_{L, T}^{2}\left|\nabla u_{L, T}\right|^{2},
$$

avec $c_{L}^{2}=\lambda+2 \mu, c_{T}^{2}=\mu$, on obtient

$$
E_{L, T}\left(u_{L, T}\right) \leqslant C E(u) .
$$

Démonstration. - Pour démontrer ce lemme, on décompose $u$ sur une base de $H_{0}^{1}(\Omega)^{3}$ formée de vecteurs propres de l'opérateur $A_{D}=-\mu \Delta-(\lambda+\mu) \nabla$ div avec conditions au bord de Dirichlet, $\left(e_{\nu}, \lambda_{\nu}\right)_{\nu \in \mathbb{N}^{*}}$ :

$$
u(t, x)=\sum_{\nu \in \mathbf{Z}^{*}} \mathrm{e}^{\operatorname{signe}(\nu) i t \sqrt{\lambda_{|\nu|}}} u_{\nu} e_{|\nu|}
$$

et on note

$$
U=A_{D}^{-1} u=\sum_{\nu \in \mathbf{Z}^{*}} \mathrm{e}^{\operatorname{signe}(\nu) i t \sqrt{\lambda_{|\nu|}}} \frac{u_{\nu}}{\lambda_{|\nu|}} e_{|\nu|} .
$$

La fonction $U$ vérifie

$$
\left(\partial_{t}^{2}+A\right) U=0
$$

et on a $U \in C^{k}\left(\mathbb{R}_{t} ; H^{3-k}(\Omega)^{3}\right), \forall k \in \mathbb{N}$. On note

$$
\begin{aligned}
& \varphi=-c_{L}^{2} \operatorname{div} U \in C^{k}\left(\mathbb{R}_{t} ; H^{2-k}(\Omega)\right), \quad \forall k \in \mathbb{N}, \\
& \psi=-c_{T}^{2} \operatorname{rot} U \in C^{k}\left(\mathbb{R}_{t} ; H^{2-k}(\Omega)^{3}\right), \quad \forall k \in \mathbb{N} .
\end{aligned}
$$

Compte tenu de (4.9), les fonctions $\varphi$ et $\psi$ vérifient respectivement $\left(\partial_{t}^{2}-c_{L}^{2} \Delta\right) \varphi=P_{L} \varphi=0$ et $\left(\partial_{t}^{2}-c_{T}^{2} \Delta\right) \psi=P_{T} \psi=0, \nabla \varphi+\operatorname{rot} \psi=-c_{L}^{2} \nabla \operatorname{div} U-c_{T}^{2} \operatorname{rot} \operatorname{rot} U=A U=-\partial_{t}^{2} U=u$. Enfin la troisième condition du lemme est triviale et la dernière est conséquence de la construction.

\subsection{Géométrie}

Soit $M=\mathbb{R}_{t} \times \Omega$. On note

$$
\left.\operatorname{Car} \mathcal{L} \subset T^{*} \mathbb{R}^{d+1}\right|_{M}=\left\{(t, \tau, z, \zeta) ;(x, t) \in \bar{M}, \tau^{2}=c_{L}^{2}|\zeta|^{2}\right\}
$$

et

$$
\left.\operatorname{Car} \mathcal{T} \subset T^{*} \mathbb{R}^{d+1}\right|_{\bar{M}}=\left\{(t, \tau, z, \zeta) ;(x, t) \in \bar{M}, \tau^{2}=c_{T}^{2} \mid \zeta^{2}\right\},
$$

les deux variétés caractéristiques des deux opérateurs des ondes. On note aussi $Z_{\mathcal{L} ; \mathcal{T}}=$ $j(\operatorname{Car} \mathcal{L} ; \mathcal{T}) \subset{ }^{b} T^{*} M$ les projections de ces deux variétés caractéristiques. Dans un système de coordonnées géodésiques où $\Delta=\partial_{x}^{2}+R\left(x, y, D_{y}\right)+Q_{1}\left(x, y, D_{y}\right)+Q_{0}\left(x, y, D_{y}\right) \partial_{x}$ où $Q_{i}$ sont d'ordre $i$, si on note $r_{0}=\left.r\right|_{x=0}$, on a

$$
Z_{\mathcal{L}, \mathcal{T}} \cap\{x=0\}=\left\{(t, y, \tau, \eta) ; \nu_{L, T}^{2} \tau^{2}+r_{0} \geqslant 0\right\},
$$


avec $\nu_{L, T}=c_{L, T}^{-1}$, et on a les décompositions

$$
T^{*} \partial M=\mathcal{H}_{L} \cup \mathcal{G}_{L} \cup \mathcal{E}_{L}=\mathcal{H}_{T} \cup \mathcal{G}_{T} \cup \mathcal{E}_{T},
$$

avec

$$
\left\{\begin{array}{l}
\mathcal{H}_{L, T}=\left\{(t, y, \tau, \eta) ; \nu_{L, T}^{2} \tau^{2}+r_{0}>0\right\}, \\
\mathcal{G}_{L, T}=\left\{(t, y, \tau, \eta) ; \nu_{L, T}^{2} \tau^{2}+r_{0}=0\right\}, \\
\mathcal{E}_{L, T}=\left\{(t, y, \tau, \eta) ; \nu_{L, T}^{2} \tau^{2}+r_{0}<0\right\} .
\end{array}\right.
$$

\subsection{Mesures}

On considère $\left(u_{k}\right)_{k \in \mathbb{N}}$ une suite d'énergie bornée de solutions du système de Lamé. On suppose que la suite $\left(u_{k}\right)$ converge faiblement vers 0 . Les suites $\left(u_{k}\right)_{L, T}$ qui lui sont associées sont des suites de solutions d'équations d'ondes $\left(\nu_{L, T}^{2} \partial_{t}^{2}-\Delta\right) u_{k, L, T}=0$ d'énergies bornées et convergent également faiblement vers 0 . Nous allons leur associer deux mesures, $\mu_{L, T}$ éléments du dual topologique de $C^{0}\left({ }^{b} S^{*} M ; \operatorname{End}\left(\mathbb{C}^{3}\right)\right)$.

\subsection{1. À l'intérieur}

Quitte à extraire une sous-suite, les procédures standard (voir P. Gérard [5] ou L. Tartar [15]) montrent qu'il existe deux mesures $\mu_{L, T}$ éléments du dual de l'espace des fonctions continues sur $S^{*} M$ à valeurs endomorphismes, telles que pour tout opérateur pseudodifférentiel $Q$ à valeurs endomorphismes de $\mathbb{C}^{3}$, de degré 0 et à support compact dans $M$, on a

$$
\left\langle\mu_{L, T}, \sigma(Q)\right\rangle=\lim _{k \rightarrow+\infty}\left(Q u_{k, L, T}, u_{k, L, T}\right)_{H_{L, T}^{1}},
$$

où on a noté

$$
\begin{aligned}
&\left(Q u_{L}, u_{L}\right)_{H_{L}^{1}}= \int_{M}\left(\partial_{t} Q u_{k, L}, \partial_{t} u_{k, L}\right)+\mu\left(\nabla Q u_{k, L}, \nabla u_{k, L}\right) \\
& \quad+(\lambda+\mu)\left(\operatorname{div} Q u_{k, L}, \operatorname{div} u_{k, L}\right), \\
&=\int_{M}\left(\partial_{t} Q u_{k, L}, \partial_{t} u_{k, L}\right)+(\lambda+2 \mu)\left(\nabla Q u_{k, L}, \nabla u_{k, L}\right)
\end{aligned}
$$

et

$$
\left(Q u_{T}, u_{T}\right)_{H_{T}^{1}}=\int_{M}\left(\partial_{t} Q u_{k, T}, \partial_{t} u_{k, T}\right)+\mu\left(\nabla Q u_{k, T}, \nabla u_{k, T}\right) .
$$

Les résultats standard de régularité elliptique montrent aussi que ces deux mesures sont portées par les variétés caractéristiques respectives, $\operatorname{Car} \mathcal{L}$ et $\operatorname{Car} \mathcal{T}$.

\subsubsection{Près d'un point du bord}

On se place dans un système de coordonnées géodésiques près d'un point $\left(x_{0}=0, y_{0}\right)$. Dans ce système $\mathrm{M}$ à la forme $X=] 0,1\left[{ }_{x} \times Y, Y=B(0,1)_{y}\right.$, le bord de $M$ a pour équation $x=0$, le laplacien $\Delta$ a la forme suivante :

$$
\Delta=\frac{1}{\sqrt{\operatorname{det} g}} \partial_{x} \sqrt{\operatorname{det} g} \partial_{x}+P\left(x, y, D_{y}\right)
$$


où $x$ désigne la variable normale au bord, $y$ la variable tangentielle, $P\left(x, y, D_{y}\right)$ est un opérateur différentiel tangentiel et la métrique $g$ est donnée par

$$
g=\left(\begin{array}{cc}
1 & 0 \\
0 & M(x, y)
\end{array}\right), \quad g^{-1}=\left(\begin{array}{cc}
1 & 0 \\
0 & \alpha(x, y)
\end{array}\right)
$$

où $\left.{ }^{t} \eta \alpha\right|_{x=0} \eta=\|\eta\|^{2}$ est la norme naturelle sur $T^{*} \partial \Omega$, duale de la norme sur $T \Omega$ induite par la métrique sur $\Omega$, et $\left.{ }^{t} \eta \partial_{x} \alpha\right|_{x=0} \eta=-2 \kappa(y, \eta)\|\eta\|^{2}$. La convention de signe que nous avons choisie est telle que $\kappa>0$ sur $\mathcal{G}^{2,+}$.

On considère les fonctions $\widetilde{u}_{L, T}=\operatorname{det} g^{1 / 4} u_{L, T}$ dans ce système de coordonnées et on note

$$
\widetilde{P}_{L, T}=\operatorname{det} g^{1 / 4} P_{L, T} \operatorname{det} g^{-1 / 4} \quad \text { où } P_{L, T}=\Delta-\nu_{L, T}^{2} \partial_{t}^{2} .
$$

Les opérateurs $\widetilde{P}_{L, T}$ sont autoadjoints pour la métrique $\mathrm{d} x|\mathrm{~d} y| \mathrm{d} t$ et de la forme $\partial_{x}^{2}+$ $\widetilde{R}_{L, T}\left(x, y, D_{y}, D_{t}\right)$, où les opérateurs $\widetilde{R}_{L, T}$ sont des opérateurs différentiels de degré 2 , tangentiels et autoadjoints pour la mesure $\mathrm{d} x|\mathrm{~d} y| \mathrm{d} t$. Le symbole principal de l'opérateur $\widetilde{R}_{L, T}$, $r_{L, T}(x, y, \eta)$, vaut

$$
r_{L, T}(x, y, \eta, \tau)=\nu_{L, T}^{2} \tau^{2}-{ }^{t} \eta \alpha(x, y) \eta
$$

ce qui implique

$$
r_{0, L, T}=\left.r\right|_{x=0}=\nu_{L, T}^{2} \tau^{2}-\|\eta\|^{2}
$$

et

$$
r_{1, L, T}=\left.\partial_{x} r\right|_{x=0}=2\|\eta\|^{2} \kappa(y, \eta) .
$$

On peut d'après la remarque 2.1, quitte à extraire une sous-suite, associer des mesures $\check{\mu}_{L, T}$ et $\check{\mu}_{\partial, L, T}^{1 / 2,0,-1 / 2}$ aux suites $\widetilde{u}_{k, L, T}$ et $\left(\left.\widetilde{u}_{k, L, T}\right|_{x=0},\left.\partial_{x} \widetilde{u}_{k, L, T}\right|_{x=0}\right)$ comme à la section 2.

LEMME 4.2. - Les mesures $\check{\mu}_{\partial, L, T}^{1 / 2,0,-1 / 2}$ sont nulles.

Démonstration. - On suppose par exemple $c_{L}>c_{T}$ (l'autre cas se traitant de la même manière). On a alors

$$
T^{*} Y=\mathcal{H}_{T} \cup \mathcal{G}_{T} \cup \mathcal{E}_{T} ; \quad \mathcal{H}_{L} \cup \mathcal{G}_{L} \subset \mathcal{H}_{T}
$$

On remarque d'abord que la condition aux limites

$$
\left.u_{k, L}\right|_{x=0}+\left.u_{k, T}\right|_{x=0}=0
$$

implique $\check{\mu}_{\partial, L}^{1 / 2}=\check{\mu}_{\partial, T}^{1 / 2}$. D'après le lemme 2.6, les mesures $\check{\mu}_{\partial, T}^{1 / 2,0,-1 / 2}$ sont portées par $\mathcal{E}_{T}$. Mais près d'un point $\rho_{0} \in \mathcal{E}_{T}$ (qui est donc elliptique pour l'opérateur $\partial_{t}^{2}+A$ ), d'après le théorème de régularité elliptique pour le problème de Dirichlet, les fonctions $u_{k}$ sont uniformément bornées dans $H^{s}$ au voisinage de $\rho_{0}$, pour tout $s \in \mathbb{R}$. D'après la construction de $u_{k, T}$ et $u_{k, L}$ à partir de $u_{k}$, il en est de même des suites $u_{k, T}$ et $u_{k, L}$, ce qui montre que les mesures $\check{\mu}_{\partial, L, T}^{1 / 2,0,-1 / 2}$ sont toutes nulles au voisinage de $\rho_{0}$. On obtient ainsi que les mesures $\check{\mu}_{\partial, T}^{1 / 2,0,-1 / 2}$ sont nulles partout. Donc la mesure $\check{\mu}_{\partial, L}^{1 / 2}=\check{\mu}_{\partial, T}^{1 / 2}$ est aussi nulle, puis en utilisant les relations (2.58) on obtient que les mesures $\check{\mu}_{\partial, L}^{0,-1 / 2}$ sont aussi nulles. 
Soient $\zeta_{L, T}$ les fonctions sur $S \widehat{Z}$ définies $\check{\mu}_{L, T}$ presque partout par

$$
\begin{cases}\zeta(x, y, \xi, \eta, \tau)=\frac{\nu_{L, T}^{2} \tau^{2}+\|\xi, \eta\|^{2}}{\tau^{2}+|\xi, \eta|^{2}} & \text { si } x>0, \\ \zeta(x, y, \xi, \eta, \tau)=\frac{\nu_{L, T}^{2} \tau^{2}+\|0, \eta\|^{2}}{\tau^{2}+|0, \eta|^{2}} & \text { si } x=0,\end{cases}
$$

où $\|\xi, \eta\|^{2}=\xi^{2}+{ }^{t} \eta A(x, y) \eta,|\xi, \eta|^{2}=\xi^{2}+{ }^{t} \eta \eta$. On définit alors la mesure $\mu_{L, T}$ comme étant la mesure qui, dans ce système de coordonnées vaut

$$
\mu_{L, T}=\zeta(x, y, \xi, \eta, \tau) \check{\mu}_{L, T} .
$$

Le lemme suivant montre que cette multiplication $\operatorname{par} \zeta$ permet de retrouver l'énergie naturelle.

LEMME 4.3. - Pour tout opérateur pseudodifférentiel $\widetilde{Q}$ sur $X$, d'ordre 0 , tangentiel près de $\{x=0\}$ et à support compact, si on note $Q$ l'opérateur pseudodifférentiel sur $M$ qui lui correspond, on a, $\mathrm{d} L$ désignant la mesure de Lebesgue sur $\Omega$,

$$
\begin{aligned}
& \left\langle\mu_{L, T}, \kappa(\sigma(\widetilde{Q}))\right\rangle \\
& =\lim _{k \rightarrow+\infty} \int_{M} \nu_{L, T}^{2}\left(\partial_{t} Q u_{k, L, T}, \partial_{t} u_{k, L, T}\right)+\left(\nabla Q u_{k, L, T}, \nabla u_{k, L, T}\right) \mathrm{d} t \mathrm{~d} L .
\end{aligned}
$$

Démonstration. - En effet (en notant $\frac{\mathrm{d}}{\mathrm{d} n}$ le champ normal extérieur à $\Omega$ ),

$$
\begin{aligned}
I_{k} & =\int_{M} \nu_{L, T}^{2}\left(\partial_{t} Q u_{k, L, T}, \partial_{t} u_{k, L, T}\right)+\left(\nabla Q u_{k, L, T}, \nabla u_{k, L, T}\right) \mathrm{d} t \mathrm{~d} L \\
& =-\int_{M}\left(\left(\nu_{L, T}^{2} \partial_{t}^{2}+\Delta\right) Q u_{k, L, T}, u_{k, L, T}\right) \mathrm{d} t \mathrm{~d} L+\int_{\partial M} \frac{\mathrm{d}}{\mathrm{d} n} Q u_{k} \overline{u_{k}} \mathrm{~d} \sigma .
\end{aligned}
$$

Puisque les mesures $\check{\mu}_{\partial, L, T}^{1 / 2,0,-1 / 2}$ sont toutes nulles, le terme de bord tend vers 0 quand $k \rightarrow+\infty$ et

$$
\begin{aligned}
I_{k}= & -\int_{M}\left(\left(\nu_{L, T}^{2} \partial_{t}^{2}+\Delta\right) Q u_{k, L, T}, u_{k, L, T}\right) \mathrm{d} t \mathrm{~d} L+\mathrm{o}(1), \\
= & -\int_{X}\left(\left(\nu_{L, T}^{2} \partial_{t}^{2}+\Delta\right) \widetilde{Q} \operatorname{det} g^{-1 / 4} \widetilde{u}_{k, L, T}, \operatorname{det} g^{-1 / 4} \widetilde{u}_{k, L, T}\right) \\
& \times \sqrt{|\operatorname{det} g|}^{1 / 2} \mathrm{~d} t \mathrm{~d} x|\mathrm{~d} y|+\mathrm{o}(1)
\end{aligned}
$$

qui converge, d'après les lemmes 2.7 et 4.2 vers

$$
\left\langle\check{\mu}_{L, T}, \kappa(\zeta \sigma(\widetilde{Q}))\right\rangle
$$

puisqu'on a dans le système de coordonnées géodésiques normales

$$
-\sigma\left(\nu_{L, T}^{2} \partial_{t}^{2}+\Delta\right)=\nu_{L, T}^{2} \tau^{2}+\|\xi, \eta\|^{2} ; \quad \lambda^{2}=\tau^{2}+|\xi, \eta|^{2} .
$$

En utilisant une partition de l'unité, on peut ainsi définir deux mesures $\mu_{L, T}$ éléments du dual de l'espace des fonctions continues sur $S \widehat{Z}_{\mathcal{L}, \mathcal{T}}$ à valeurs endomorphismes de $\mathbb{C}^{3}$, vérifiant pour 
tout $Q$, opérateur pseudodifférentiel sur $M$ d'ordre 0 , tangentiel (ou plus précisément somme d'opérateurs qui sont tangentiels dans des systèmes de coordonnées géodésiques),

$$
\begin{aligned}
& \left\langle\mu_{L, T}, \kappa(\sigma(\widetilde{Q}))\right\rangle \\
& \quad=\lim _{k \rightarrow+\infty} \int_{M} \nu_{L, T}^{2}\left(\partial_{t} Q u_{k, L, T}, \partial_{t} u_{k, L, T}\right)+\left(\nabla Q u_{k, L, T}, \nabla u_{k, L, T}\right) \mathrm{d} t \mathrm{~d} L .
\end{aligned}
$$

On identifiera par la suite ces deux mesures aux mesures correspondantes définies sur le dual de l'espace des fonctions continues sur ${ }^{b} S^{*} M\left({ }^{b} T^{*} M\right.$ quotienté par l'action naturelle de $\left.\mathbb{R}^{+}\right)$, à valeurs endomorphismes de $\mathbb{C}^{3}$. On remarquera que d'après (4.34), $u$ est une section du fibré tangent à $\Omega$ et la fibre $\mathbb{C}^{3}$ est l'espace tangent en chaque point à $\Omega$. Enfin, d'après les lemmes $2.7,4.2$ et 4.3 , si $Q$ est un opérateur pseudo-différentiel sur $M$, d'ordre 2 , qui près du bord est de la forme $Q=\sum_{j=0}^{2} Q_{2-j} \partial_{x}^{j}$, avec $Q_{i}$ tangentiels de degrés $i$, en remarquant que $Q u_{k, L, T}=\left(\sum_{j=0}^{1} Q_{2-j} \partial_{x}^{j}-Q_{0} \tilde{R}_{L, T}\right) u_{k, L, T}$, on obtient

$$
\lim _{k \rightarrow+\infty} \int_{M}\left(Q u_{k, L, T}, u_{k, L, T}\right) \mathrm{d} t \mathrm{~d} L=\left\langle\mu_{L, T}, \frac{q}{\nu_{L, T}^{2} \tau^{2}+\|\xi, \eta\|^{2}}\right\rangle,
$$

où $q$ est le symbole principal de $Q$.

Proposition 4.4. - Les mesures $\mu_{L}$ et $\mu_{T}$ sont mutuellement singulières (au sens de la théorie de la mesure).

Démonstration. - À l'intérieur ce résultat est immédiat puisque les mesures $\mu_{L}$ et $\mu_{T}$ sont portées par $j(\operatorname{Car} \mathcal{L})$ et $j(\operatorname{Car} \mathcal{T})$ respectivement (et ces deux ensembles sont disjoints). Il suffit donc de montrer la proposition en remplaçant $\mu_{L, T}$ par $\mu_{L, T} 1_{\partial M}$. Près d'un point du bord, $\rho_{0}$, on suppose par exemple $c_{L}>c_{T}$. Si $\rho_{0} \in \mathcal{H}_{T}$, alors la proposition est vérifiée près de $\rho_{0}$ puisque $\mu_{T} 1_{\mathcal{H}_{T}}=0$ d'après le lemme 2.6. Si $\rho_{0} \in \mathcal{E}_{T}$ alors $\rho_{0}$ est elliptique pour l'opérateur $\partial_{t}^{2}+A$ et les résultats standard de régularité elliptique pour le problème de Dirichlet montrent que $u_{k}$ (et donc aussi $u_{k, L}$ et $u_{k, T}$ ) est uniformément bornée dans $H^{s}$ pour tout $s$, donc $\mu_{L}$ et $\mu_{T}$ sont nulles près de $\rho_{0}$. Enfin, si $\rho_{0} \in \mathcal{G}_{T} \subset \mathcal{E}_{L}$, comme $\mu_{\partial, L}^{1 / 2}=0$, la régularité elliptique pour l'opérateur $\nu_{L}^{2} \partial_{t}^{2}-\Delta$ avec conditions de Dirichlet montre que, près de $\rho_{0}, u_{k, L}$ converge fortement vers 0 dans $H^{1}$ donc $\mu_{L}=0$.

\subsubsection{Polarisation}

Nous n'étudions ici que la polarisation de la mesure $\mu_{T}$ puisque c'est celle dont nous nous servirons ultérieurement, néanmoins il est clair qu'un résultat analogue est vrai pour la mesure $\mu_{L}$. Soit $\Pi(x, \xi)$ l'opérateur égal à l'intérieur ou en un point du bord $\rho_{0} \in \mathcal{G}_{T}$ au projecteur orthogonal sur le plan orthogonal à $\xi$. Cet opérateur est défini $\mu_{T}$ presque partout et vaut $\Pi=\mathrm{Id}-\Pi^{\perp}$ avec

$$
\forall X \in \mathbb{C}^{3}, \quad \Pi^{\perp}(x, \varrho)(X)=\frac{\langle\varrho, X\rangle}{\|\varrho\|^{2}} g^{-1} \varrho .
$$

Lemme 4.5. - Pour tout $q \in C^{0}\left({ }^{b} S^{*} M ; \operatorname{End}\left(\mathbb{C}^{3}\right)\right)$

$$
\left\langle\mu_{T}, q\right\rangle=\left\langle\mu_{T}, \Pi q\right\rangle=\left\langle\mu_{T}, q \Pi\right\rangle=\left\langle\mu_{T}, \Pi q \Pi\right\rangle .
$$


Démonstration. - Soit $B$ un opérateur pseudo-différentiel de degré 0 , tangentiel près du bord, de symbole principal $b$. On peut appliquer (4.35) à $Q=B \nabla \operatorname{div}$, d'où en utilisant $\operatorname{div} u_{T}=0$,

$$
\left\langle\mu_{T}, \frac{\|\xi, \eta\|^{2}}{\nu_{T}^{2} \tau^{2}+\|\xi, \eta\|^{2}} b \Pi^{\perp}\right\rangle=0
$$

ce qui implique la première relation de (4.37). La deuxième s'obtient à partir de la première puisque la mesure $\mu_{T}$ est hermitienne et la troisième est conséquence des deux premières.

\section{Le théorème de propagation dans le cas où $\mu_{L}=0$}

On suppose ici que $\operatorname{div} u_{k}=\operatorname{div} u_{k, L}$ converge vers 0 dans $L^{2}(] 0, T[\times \Omega)$ quand $t \rightarrow+\infty$. Nous allons démontrer qu'alors la mesure $\mu_{T}$ est invariante par un certain flot qu'on explicitera. On commence par montrer que $\mu_{L}$ est nulle. On a $-\operatorname{div} u_{k, L}=c_{L}^{2} \operatorname{div} \nabla \operatorname{div} U_{k}=c_{L}^{2} \Delta \operatorname{div} U_{k}$. En dehors de la variété caractéristique de l'opérateur $\partial_{t}^{2}+A,\left(U_{k}\right)$ converge fortement vers 0 dans tous les espaces $H^{s}$ et près de la variété caractéristique, l'opérateur $\partial_{t}^{2}$ est elliptique, tangentiel et on a $\partial_{t}^{2} \operatorname{div} U_{k}=c_{L}^{2} \Delta \operatorname{div} U_{k}=-\operatorname{div} u_{k, L} \rightarrow 0$ dans $L^{2}$. D'après la régularité elliptique, $\operatorname{div} U_{k} \rightarrow 0$ dans $H^{2}$ et donc $u_{k, L}=-c_{L}^{2} \nabla \operatorname{div} U_{k} \rightarrow 0$ dans $H^{1}$, soit $\mu_{L}=0$.

On rappelle que le support de $\mu_{T}$ est inclus dans la projection de la variété caractéristique $Z_{\mathcal{T}}$. Puisque les fonctions $u_{k, T}$ sont solutions de l'équation $\left(\partial_{t}^{2}-c_{T}^{2} \Delta\right) u_{k, T}=0$, il est standard que la mesure $\mu_{T}$ est près de tout point intérieur $\rho_{0} \in Z_{\mathcal{T}} \cap T^{*} M$ invariante par le flot du champ Hamiltonien homogène $H_{p_{T} / \tau}$.

\subsection{Près d'un point $\rho_{0} \in \mathcal{H}_{L}$}

Près d'un point hyperbolique pour l'onde longitudinale les résultats standard de régularité pour les solutions de l'équation des ondes montrent, puisque $u_{k, L}$ tend vers 0 dans $H_{\varrho_{0}}^{1}$,

$$
\left\|\left.u_{k, L}\right|_{\partial M}\right\|_{H_{\rho_{0}}^{1}} \leqslant C\left\|u_{k, L}\right\|_{H_{\rho_{0}}^{1}}
$$

et

$$
\left\|\left.\partial_{n} u_{k, L}\right|_{\partial M}\right\|_{L_{\rho_{0}}^{2}} \leqslant C\left\|u_{k, L}\right\|_{H_{\rho_{0}}^{1}} .
$$

Les deux traces de $u_{k, L}$ convergent donc fortement vers 0 dans $H^{1}$ et $L^{2}$ respectivement près de $\rho_{0}$. Près de $\rho_{0}, u_{k, T}$ vérifie donc des conditions au bord de Dirichlet :

$$
\begin{aligned}
& \left(\partial_{t}^{2}-c_{T}^{2} \Delta\right) u_{k, T}=0, \\
& \left.u_{k, T}\right|_{\partial M}=-\left.u_{k, L}\right|_{\partial M}=\mathrm{o}(1)_{H_{\varrho_{0}}^{1}} .
\end{aligned}
$$

Le théorème de propagation de la mesure de la section 3 montre alors que $\mu_{T}$ est invariante par le flot de Melrose et Sjöstrand (ici la trivialisation du fibré $\Sigma / \mathbb{R}_{+}^{*} \times \mathbb{C}^{3}$ est celle qui est donnée par la relation (3.16), pour $J_{\xi}=\mathrm{Id}$, avec $M(s, \rho)=\mathrm{Id}$ ). On note $\xi_{ \pm}$les deux points (éventuellement confondus) de la variété caractéristique qui se projettent sur $\rho$ et $\Pi_{ \pm}$le projecteur orthogonal sur le plan orthogonal à $\xi_{ \pm}$. On notera encore $\Pi_{ \pm}$les transportés par le flot de ces projecteurs et $\Pi_{+,-}$le projecteur orthogonal sur l'espace orthogonal à l'espace engendré par $\xi_{+}$et $\xi_{-}$. D'après le théorème de propagation près de $\rho_{0}$ et le lemme 4.5 , près de $\rho_{0}$, on a

$$
\mu_{T}=\Pi_{+} \mu_{T} \Pi_{+}=\Pi_{-} \mu_{T} \Pi_{-}=\Pi_{+,-} \mu_{T} \Pi_{+,-} .
$$


On remarquera que, sauf dans le cas d'un rayon normal ou tangent au bord, cette information est non triviale puisqu' alors $\Pi_{+,-}$est le projecteur orthogonal sur la droite normale à la fois à la direction incidente et à la normale au bord en $\rho_{0}$.

\subsection{Près d'un point $\rho_{0} \in \mathcal{E}_{L}$}

Ce cas est non trivial uniquement dans le cas où $c_{L}>c_{T}$. En effet dans le cas contraire, $Z_{\mathcal{T}} \cap \mathcal{E}_{L}=\emptyset$ et la mesure $\mu_{T}$ est nulle près de $\rho_{0}$. Pour l'étude on se place dans un système de coordonnées géodésiques près de $\rho_{0}$. On note $\chi: M \rightarrow X$ le changement de variables correspondant. Soit $v_{k, T}$ le champ de vecteurs sur $X, v_{k, T}=T \chi\left(u_{k, T}\right)$. On calcule le système de conditions au bord vérifié par les fonctions $v_{k, T}$ (on en déduira simplement celui vérifié par $\left.\widetilde{u}_{k, T}\right)$. Les fonctions $\varphi_{k}$ associées à $\left(u_{k}\right)$ par le lemme 4.1 vérifient $P_{L} \varphi_{k}=0$ et le point $\rho_{0}$ est elliptique pour l'opérateur $P_{L}$. Il existe donc, d'après la théorie elliptique, un opérateur pseudodifférentiel, $E_{-1}\left(y, D_{y}\right)$, d'ordre -1 tel que près de $\rho_{0}$,

$$
\left.\varphi_{k}\right|_{\partial M}=E_{-1}\left(\left.\frac{\partial \varphi_{k}}{i \partial x}\right|_{\partial M}\right)
$$

et le symbole principal $e_{-1}$ de $E_{-1}$ vaut

$$
e_{-1}(y, \eta)=\frac{-i}{\sqrt{\|\eta\|^{2}-\tau^{2} \nu_{L}^{2}}}
$$

Les conditions aux limites vérifiées par les trois composantes du vecteur $v_{k, T}$ sont

$$
\begin{aligned}
\left.v_{k, T, x}\right|_{\partial M} & =-\left.\partial_{x} \varphi\right|_{\partial M} \\
\left(\begin{array}{c}
v_{k, T, y_{1}} \\
v_{k, T, y_{2}}
\end{array}\right) & =-\alpha\left(\begin{array}{c}
\left.\partial_{y_{1}} \varphi\right|_{\partial M} \\
\left.\partial_{y_{2}} \varphi\right|_{\partial M}
\end{array}\right) .
\end{aligned}
$$

La condition $\operatorname{div} u_{k, T}=0$ s'écrit $\operatorname{div}_{g} v_{k, T}=0$, soit

$$
\partial_{x} v_{k, T, x}+\partial_{y_{1}} v_{k, T, y_{1}}+\partial_{y_{2}} v_{k, T, y_{2}}=\mathcal{O}(1)_{H_{\rho_{0}}^{1}} .
$$

D'après (5.7) (5.8) et (5.9), on obtient près de $\rho_{0}$,

$$
\left.v_{k, T, x}\right|_{\partial M}=Q_{-1}\left(\left.\frac{\partial v_{k, T, x}}{i \partial x}\right|_{\partial M}\right)+\mathrm{o}(1)_{\partial, H_{\rho_{0}}^{1}}
$$

où $Q_{-1}$ est un opérateur pseudodifférentiel d'ordre -1 , elliptique de symbole principal

$$
q_{-1}(y, \eta)=\frac{-i \sqrt{\|\eta\|^{2}-\tau^{2} \nu_{L}^{2}}}{\|\eta\|^{2}} .
$$

Reportant cette relation dans (5.8) et en utilisant (5.7) on obtient aussi

$$
\left(\begin{array}{l}
v_{k, T, y_{1}} \\
v_{k, T, y_{2}}
\end{array}\right)=-\alpha\left(\begin{array}{c}
\partial_{y_{1}} \\
\partial_{y_{2}}
\end{array}\right) E_{-1} Q_{-1}\left(\left.\partial_{x} v_{k, T, x}\right|_{\partial M}\right)+\mathrm{o}(1)_{\partial, H_{\rho_{0}}^{1}} .
$$

Finalement le système de conditions aux limites que $v_{k, T}$ vérifie est de la forme

$$
v_{k, T}=B_{-1}^{\prime}\left(\frac{\partial v_{k, T}}{i \partial x}\right)+\mathrm{o}(1)_{\partial, H_{\rho_{0}}^{1}}
$$


où $B_{-1}^{\prime}$ est une matrice $3 \times 3$ d'opérateurs pseudodifférentiels d'ordre -1 de symbole principal

$$
b_{-1}^{\prime}(y, \eta)=\left(\begin{array}{ccc}
\frac{-i \sqrt{\|\eta\|^{2}-\tau^{2} \nu_{L}^{2}}}{\|\eta\|^{2}} & 0 & 0 \\
-\frac{\alpha}{\|\eta\|^{2}}\left(\begin{array}{l}
\eta_{1} \\
\eta_{2}
\end{array}\right) & 0 & 0 \\
0 & 0
\end{array}\right) .
$$

Si on revient aux conditions aux limites vérifiées par $\widetilde{u}_{k, T}=(\operatorname{det} g)^{1 / 4} u_{k, T}$, on obtient

$$
\widetilde{u}_{k, T}=B_{-1}\left(\frac{\partial \widetilde{u}_{k, T}}{i \partial x}\right)+\mathrm{o}(1)_{\partial, H_{\rho_{0}}^{1}}
$$

où $B_{-1}=T \chi^{-1} B_{-1}^{\prime} T \chi$. Nous nous trouvons donc exactement dans le cadre d'application du théorème de propagation de la mesure de la section 3. Il ne reste donc qu'à identifier les objets géométriques qui interviennent dans la définition du flot : les endomorphismes $J_{\xi}$ et $M(s)$. A priori le théorème de propagation décrit la propagation de la mesure $\check{\mu}_{T}$ (dont on déduit la propagation de la mesure $\mu_{T}=\zeta \check{\mu}_{T}$ ). Évidemment, l'endomorphisme $J_{\xi}$ est le même pour les deux mesures. En ce qui concerne $M(s)$, il est plus simple de calculer directement l'endomorphisme associé à la mesure $\mu_{T}$.

\subsubsection{L'opérateur $J_{\xi}$}

Calculons l'opérateur $J_{\xi}$ dans la base $\left(\partial_{x}, \partial_{y_{1}}, \partial_{y_{2}}\right)$, c'est à dire la matrice $J_{\xi}^{\prime}$ :

$$
J_{\xi}^{\prime}=T \chi J_{\xi} T \chi^{-1}
$$

On a $J_{\xi}^{\prime}=\left(I d-\xi b_{-1}^{\prime}\right)^{-1}\left(I d+\xi b_{-1}^{\prime}\right)$, soit

$$
J_{\xi}^{\prime}=\left(\begin{array}{ccc}
\frac{\|\eta\|^{2}-\xi \delta}{\|\eta\|^{2}+\xi \delta} & 0 & 0 \\
\frac{-2 \xi}{\|\eta\|^{2}+\xi \delta} \alpha\left(\begin{array}{l}
\eta_{1} \\
\eta_{2}
\end{array}\right) & 1 & 0 \\
0 & 1
\end{array}\right),
$$

avec $\delta=i \sqrt{\|\eta\|^{2}-\tau^{2} \nu_{L}^{2}}$. Compte tenu de ce que la mesure $\mu_{T}$ est polarisée perpendiculairement à la direction de propagation $(\xi, \eta)$, on s'intéresse juste à son action sur le plan $(\xi, \eta)^{\perp}$. Une base de ce plan est donnée par les vecteurs

$$
e_{1}(\xi)=(0, Y), \quad e_{2}(\xi)=\left(\|\eta\|^{2},-\alpha(\eta) \xi\right)
$$

où $Y$ est un vecteur de l'espace tangent $T Y$, tel que $\langle Y, \eta\rangle=0$. On a donc

$$
\begin{aligned}
J_{\xi}^{\prime}\left(e_{1}(-\xi)\right) & =e_{1}(\xi), \\
J_{\xi}^{\prime}\left(e_{2}(-\xi)\right) & =\frac{\|\eta\|^{2}-\xi \delta}{\|\eta\|^{2}+\xi \delta} e_{2}(\xi) .
\end{aligned}
$$

Puisque $\delta \in i \mathbb{R}$, l'endomorphisme $-J_{\xi}$ qui traduit la réflexion hyperbolique est donc une isométrie de $(-\xi, \eta)^{\perp} \operatorname{sur}(\xi, \eta)^{\perp}$ (ce caractère isométrique traduit la conservation de l'énergie). Les relations (5.19), (5.20) nous permettent de retrouver la relation (5.30) de [11] (calculée par des arguments d'optique géométrique). 


\subsubsection{L'endomorphisme $M(s)$}

La description précédente de l'endomorphisme $J_{\xi}$ caractérise la propagation de la mesure $\mu_{T}$ le long du rayon issu du point $\rho_{0} \in \mathcal{E}_{L}$, sauf si ce rayon est un rayon glissant près de $\rho_{0}$ $\left(\gamma(s) \in \mathcal{G}^{2,-}\right.$ pour $\left.\left.\left.\pm s \in\right] 0, \varepsilon\right]\right)$. Pour terminer la description du cas $\rho_{0} \in \mathcal{E}_{L}$, il nous suffit donc de décrire l'endomorphisme $M(s)$, le long d'un rayon glissant. Puisque l'opérateur de Laplace est autoadjoint, la mesure $\mu_{T}$ vérifie pour tout $q \in C_{\text {paire }}^{\infty, 1}\left(p^{-1}(0) ;\right.$ End $\left.\mathbb{C}^{3}\right)$

$$
\left\langle\mu_{T}, \frac{\{p, q\}}{\lambda^{2}}\right\rangle=0,
$$

avec

$$
\lambda^{2}=\zeta\left(\tau^{2}+|\eta|^{2}\right),\left.\quad \lambda^{2}\right|_{\mathcal{G}}=\nu_{T}^{2} \tau^{2}+\left\|\eta^{2}\right\|
$$

L'endomorphisme $m$ de la relation (2.98) est donc nul. L'endomorphisme $n$ de la relation (3.9) vaut donc

$$
n=i\left\{p, J_{\xi}^{-1 / 2}\right\} J_{\xi}^{1 / 2},
$$

or $\left.J_{\xi}\right|_{\mathcal{G}}=\mathrm{Id}$, donc

$$
\left.n\right|_{\mathcal{G}}=-\frac{i}{2}\left\{p, J_{\xi}\right\}=\left.i \frac{\partial p}{\partial x} b_{-1}\right|_{\mathcal{G}}
$$

D'après (5.23), l'endomorphisme $l$ de la relation (3.11) vaut donc dans la base $\partial_{x}, \partial_{y_{1}}, \partial_{y_{2}}$, et en se restreignant à $\tau>0$

$$
\begin{aligned}
l & =\frac{1}{2} \frac{\tau}{\lambda^{2}}\left\{\frac{p}{\tau}, \frac{\lambda^{2}}{\tau}\right\}-i \frac{n}{\tau}=-i \frac{n}{\tau}, \\
& =\frac{2 \kappa(y, \eta)}{\tau}\left(\begin{array}{ccc}
-\delta & 0 & 0 \\
-\alpha\left(\begin{array}{c}
\eta_{1} \\
\eta_{2}
\end{array}\right) & 0 & 0 \\
0 & 0
\end{array}\right) .
\end{aligned}
$$

Calculons l'action de l'opérateur $l$ sur le trièdre de Frénet $(t, n, b)$ le long d'une bicaractéristique $\gamma$. Comme $\gamma$ est un rayon glissant, sa projection spatiale est une géodésique du bord pour la métrique induite, $t$ est le vecteur tangent à la projection spatiale de $\gamma, n$ est la normale intérieure au bord et $b=t \wedge n$. Notre choix de paramétrage donne le long de la bicaractéristique

$$
\frac{\mathrm{d}}{\mathrm{d} s} y=-2 \frac{\alpha\left(\begin{array}{l}
\eta_{1} \\
\eta_{2}
\end{array}\right)}{\tau}
$$

et en notant $\kappa=\kappa(y, \eta)$,

$$
\left\{\begin{array}{l}
n=\left(1_{x}, 0_{y}\right), \\
t=\left(0, \frac{\alpha(\eta)}{\|\eta\|}\right), \\
l(t)=0, \\
l(b)=0, \\
l(n)=\frac{-2 \kappa}{\tau}(\delta n+\alpha(\eta)) .
\end{array}\right.
$$

Or $\operatorname{sur} \mathcal{G}, \nu_{T}^{2} \tau^{2}=\|\eta\|^{2}$, donc

$$
l(n)=-\frac{2 \kappa}{\tau} \delta n+2 \kappa \nu_{T} t
$$


La géodésique du bord est paramétrée à vitesse $2 \nu_{T}$. En effet

$$
\left\|\frac{\mathrm{d}}{\mathrm{d} s} y\right\|=\|2 \alpha(\eta)\| / \tau=2 \nu_{T} .
$$

Les dérivées du trièdre de Frénet valent donc :

$$
\frac{\mathrm{d}}{\mathrm{d} s}\left(\begin{array}{c}
t \\
n \\
b
\end{array}\right)=2 \nu_{T}\left(\begin{array}{ccc}
0 & -\kappa & 0 \\
\kappa & 0 & -\zeta \\
0 & \zeta & 0
\end{array}\right)\left(\begin{array}{l}
t \\
n \\
b
\end{array}\right),
$$

où $|\kappa|$ est la courbure et $\zeta$ la torsion de la géodésique vue comme une courbe de $\mathbb{R}^{3}$. On remarquera qu'avec nos conventions, $\kappa<0$ sur $\mathcal{G}^{2,-}$.

Soient $(\alpha(s), \beta(s), \gamma(s))$ les solutions du système où $M$ est défini par la relation (3.12)

$$
(\alpha t+\beta n+\gamma b)=M(s)\left(\alpha_{0} t_{0}+\beta_{0} n_{0}+\gamma_{0} b_{0}\right),
$$

qui vérifient donc

$$
\begin{aligned}
\dot{\alpha} t & +\dot{\beta} n+\dot{\gamma} b-2 \nu_{T} \alpha(\kappa n)+2 \nu_{T} \beta(\kappa t-\zeta b)+2 \nu_{T} \gamma(\zeta n) \\
& =\beta\left(-\frac{2 \kappa}{\tau} \delta n+2 \kappa \nu_{T} t\right),
\end{aligned}
$$

soit

$$
\left\{\begin{array}{l}
\dot{\alpha}=2 \nu_{T} \kappa \beta-2 \kappa \nu_{T} \beta=0, \\
\dot{\beta}=2 \nu_{T} \kappa \alpha-2 \nu_{T} \zeta \gamma-\frac{2 \kappa}{\tau} \delta \beta, \\
\dot{\gamma}=2 \nu_{T} \zeta \beta .
\end{array}\right.
$$

On ne s'intéresse qu'à des ondes polarisées perpendiculairement à la direction de propagation, on vérifie donc que si $\alpha_{0}=0$ alors $\alpha(s)=0$, ce qui correspond à la première équation. L'opérateur $M(s)$ restreint au plan engendré par $n$ et $b$ est donc décrit par le système :

$$
\begin{aligned}
& \dot{\beta}=-2 \nu_{T} \zeta \gamma-\frac{2 \kappa}{\tau} \delta \beta, \\
& \dot{\gamma}=2 \nu_{T} \zeta \beta,
\end{aligned}
$$

dont on vérifie qu'il est bien une isométrie de ce plan (ceci correspond à la conservation de l'énergie), puisque la matrice $\left(\begin{array}{cc}-2 \kappa \delta / \tau & -2 \nu_{T} \zeta \\ 2 \nu_{T} \zeta & 0\end{array}\right)$ est anti-adjointe (car $\delta$ est imaginaire pur).

\subsection{Près d'un point $\rho_{0} \in \mathcal{G}_{L}$}

On se place dans un système de coordonnées géodésiques près du point $x_{0}$. On notera $\chi$ le changement de variables correspondant et $J_{\xi}=T \chi^{-1} J_{\xi}^{\prime} T \chi$ l'opérateur défini près de $\varrho_{0}$ dans $S^{*} Y \operatorname{par}(5.17)$ où

$$
\delta= \begin{cases}i \sqrt{\|\eta\|^{2}-\tau^{2} \nu_{L}^{2}} & \text { si } \varrho \in \mathcal{E}_{L}, \\ \sqrt{\tau^{2} \nu_{L}^{2}-\|\eta\|^{2}} & \text { si } \varrho \in \mathcal{H}_{L} \cup \mathcal{G}_{L} .\end{cases}
$$

On notera $C_{\text {paire }}^{\infty, 1}\left(p^{-1}(0)\right.$; End $\left.\mathbb{C}^{3}\right)$ l'espace des fonctions $q C^{\infty}$ homogènes de degré 1 , de $p^{-1}(0)$ à valeurs dans $\mathbb{C}^{3}$ à support près de $\left(x_{0}=0, \xi= \pm \xi_{0}, y_{0}, \eta_{0}\right)$ et vérifiant la condition 
de symétrie

$$
J_{\xi}^{*} q(x=0, \xi, y, \eta) J_{\xi}=q(x=0,-\xi, y, \eta) .
$$

Proposition 5.1. - Soit $q \in C_{\text {paire }}^{\infty, 1}\left(p^{-1}(0) ;\right.$ End $\left.\mathbb{C}^{3}\right)$. Alors, on a

$$
\left\langle\mu_{T}, \frac{\{p, q\}}{\lambda^{2}}\right\rangle=0
$$

où $\lambda$ est la fonction définie en (5.22).

COROLlaire 5.2. - La mesure $\mu_{T}$ est, près de $\varrho_{0}$, invariante par le flot associé à la réflexion hyperbolique décrite par $J_{\xi}$.

Puisque la mesure $\mu_{T}$ est polarisée perpendiculairement à la direction de propagation, il suffit pour décrire complètement sa propagation de décrire l'action de $J_{\xi}$ sur le plan perpendiculaire à $(\xi, \eta)$, qui est la même que celle des relations (5.19) et (5.20), avec notre nouvelle définition de $\delta$. On remarquera que cette description est, dans la région $\mathcal{H}_{L}$ compatible avec la description de la section 5.1 (celle correspondant aux conditions aux limites de Dirichlet), puisqu'alors on sait que la mesure $\mu_{T}$ est polarisée le long de $e_{1}$ et que les deux descriptions coïncident alors.

Démonstration de la proposition 5.1. - Si $\varrho_{0} \in \mathcal{E}_{T}$, d'après le lemme 4.2, $\left.u_{k, T}\right|_{\partial M}$ converge fortement vers 0 dans $H^{1 / 2}$, d'après les résultats standard de régularité elliptique, on en déduit que $u_{k, T}$ converge fortement vers 0 dans $H^{1}$ près de $\varrho_{0}$. Si $\varrho_{0} \in \mathcal{H}_{T}$. Les traces $\widetilde{u}_{k, T}$ et $\partial_{x} \widetilde{u}_{k, T}$ sont donc bornées dans $H_{\varrho_{0}}^{1}$ et $L_{\varrho_{0}}^{2}$ respectivement. On peut donc leur associer une mesure

$$
\mu_{\partial}=\left(\begin{array}{cc}
\mu_{\partial}^{1} & \left(\mu_{\partial}^{1 / 2}\right)^{*} \\
\mu_{\partial}^{1 / 2} & \mu_{\partial}^{0}
\end{array}\right)
$$

sur $S^{*} Y$ caractérisée par

$$
\left\{\begin{array}{l}
\forall Q \in \mathcal{A}_{I}^{1}, \quad \lim _{k} \int_{\partial X} Q \widetilde{u}_{T}^{k} \cdot \overline{\partial_{x} \widetilde{u}_{T}^{k}} \mathrm{~d} y=\left\langle\mu_{\partial}^{1 / 2},\left.\frac{\sigma(Q)}{|\eta|}\right|_{x=0}\right\rangle, \\
\forall Q \in \mathcal{A}_{I}^{2}, \quad \lim _{k} \int_{\partial X} Q \widetilde{u}_{T}^{k} \cdot \overline{\widetilde{u}_{T}^{k}} \mathrm{~d} y=\left\langle\mu_{\partial}^{1},\left.\frac{\sigma(Q)}{|\eta|^{2}}\right|_{x=0}\right\rangle, \\
\forall Q \in \mathcal{A}_{I}^{0}, \quad \lim _{k} \int_{\partial X} Q \partial_{x} \widetilde{u}_{T}^{k} \cdot \overline{\partial_{x} \widetilde{u}_{T}^{k}} \mathrm{~d} y=\left\langle\mu_{\partial}^{0},\left.\sigma(Q)\right|_{x=0}\right\rangle .
\end{array}\right.
$$

Soit $\widetilde{\mu}_{T}=\mu_{T} / \zeta$ la mesure associée dans notre système de coordonnées à la suite $\widetilde{u}_{k, T}$. On a, avec $\lambda_{0}^{2}=\tau^{2}+|\xi, \eta|^{2}$,

$$
\left\langle\mu_{T}, \frac{\{p, q\}}{\lambda^{2}}\right\rangle=\left\langle\widetilde{\mu}_{T}, \frac{\{p, q\}}{\lambda_{0}^{2}}\right\rangle .
$$

Soit $Q=Q_{0}\left(\frac{\partial_{x}}{i}\right)+Q_{1}$ où $Q_{i}$ sont des opérateurs pseudodifférentiels tangentiels d'ordre $i$ à supports proches de $\varrho_{0}$. On a

$$
\begin{aligned}
\left(i\left(\widetilde{P}_{T}^{*} Q-Q \widetilde{P}_{T}\right) \widetilde{u}_{T, k}, \widetilde{u}_{T, k}\right)_{L^{2}}= & \int_{Y}-Q_{0} \partial_{x}^{2} \widetilde{u}_{T, k} \overline{\widetilde{u}_{T, k}}-i Q_{1} \partial_{x} \widetilde{u}_{T, k} \overline{\widetilde{u}_{T, k}} \\
& +i Q_{1} \widetilde{u}_{T, k} \overline{\partial_{x} \widetilde{u}_{T, k}}+Q_{0} \partial_{x} \widetilde{u}_{T, k} \overline{\partial_{x} \widetilde{u}_{T, k}}+o(1) .
\end{aligned}
$$


On en déduit que

$$
\left\langle\widetilde{\mu}_{T}, \frac{\left\{p, q_{0} \xi+q_{1}\right\}}{\lambda_{0}^{2}}\right\rangle=\left\langle\left(\begin{array}{cc}
\mu_{\partial}^{1} & \left(\mu_{\partial}^{1 / 2}\right)^{*} \\
\mu_{\partial}^{1 / 2} & \mu_{\partial}^{0}
\end{array}\right),\left(\begin{array}{cc}
q_{0} r_{T} & -i q_{1} \\
i q_{1} & q_{0}
\end{array}\right)\right\rangle .
$$

Pour $q \in C^{\infty, 1}\left(p^{-1}(0)\right.$; End $\left.\mathbb{C}^{3}\right)$, il existe deux opérateurs tangentiels $Q_{i}$ tels que $q=q_{0} \xi+q_{1}$. En utilisant le théorème de division de Malgrange, on montre comme à la preuve du lemme 2.9 que la relation (5.41) reste vraie pour un tel symbole. Il suffit donc pour conclure de vérifier que si $q$ est paire, alors le terme de droite dans (5.41) est nul. Puisque $\widetilde{u}_{k, T}$ vérifie sur $\mathcal{E}_{L}$ la condition aux limites (5.15), le lemme 2.10 montre que ce terme est nul sur $\mathcal{E}_{L}$. La fonction $u_{k, T}$ vérifie par ailleurs sur $\mathcal{H}_{L}$ la condition aux limites $\left.u_{k, T}\right|_{\partial M}=\mathrm{o}(1)_{H_{\varrho_{0}}^{1}}$ et $v_{k, T}=T \chi u_{k, T}$ vérifie $\operatorname{div}_{g} v_{k, T}=0$, donc

$$
\partial_{x} v_{k, T, x}+\partial_{y_{1}} v_{k, T, y_{1}}+\partial_{y_{2}} v_{k, T, y_{2}}=\mathrm{o}(1)_{L_{\varrho_{0}}^{2}}
$$

ce qui implique $\left.\partial_{x} v_{k, T, x}\right|_{\partial M}=\mathrm{o}(1)_{L_{\varrho_{0}}^{2}}$ et en particulier $v_{k, T}$ vérifie aussi sur $\mathcal{H}_{L}$ la condition aux limites (5.13), où on remplace $i \sqrt{\|\eta\|^{2}-\tau^{2} \nu_{L}^{2}}$ par $\sqrt{\tau^{2} \nu_{L}^{2}-\|\eta\|^{2}}$ (et donc $\widetilde{u}_{k, T}$ vérifie la condition (5.15)). Le terme de droite dans (5.41) est donc aussi nul sur $\mathcal{H}_{L}$. Il est donc égal à

$$
\left\langle 1_{\mathcal{G}_{L}}\left(\begin{array}{cc}
\mu_{\partial}^{1} & \left(\mu_{\partial}^{1 / 2}\right)^{*} \\
\mu_{\partial}^{1 / 2} & \mu_{\partial}^{0}
\end{array}\right),\left(\begin{array}{cc}
q_{0} r_{T} & -i q_{1} \\
i q_{1} & q_{0}
\end{array}\right)\right\rangle .
$$

Soient $\widetilde{q}_{i}=\left(T \chi^{-1}\right)^{*} q_{i} T \chi^{-1}$ et $\widetilde{\mu}_{\partial}$ définie par

$$
\left\langle\widetilde{\mu}_{\partial}, \widetilde{q}\right\rangle=\left\langle\mu_{\partial}, q\right\rangle .
$$

Il nous faut donc montrer que, si $\widetilde{q}=\widetilde{q}_{0} \xi+\widetilde{q}_{1}$ et $\widetilde{q}$ paire, c'est-à-dire

$$
J_{\xi}^{\prime *} \widetilde{q}(x=0, \xi, y, \eta) J_{\xi}^{\prime}=\widetilde{q}(x=0,-\xi, y, \eta),
$$

avec

$$
J_{\xi}^{\prime}=T \chi J_{\xi} T \chi^{-1}
$$

alors

$$
\left\langle 1_{\mathcal{G}_{L}}\left(\begin{array}{cc}
\widetilde{\mu}_{\partial}^{1} & \left(\widetilde{\mu}_{\partial}^{1 / 2}\right)^{*} \\
\widetilde{\mu}_{\partial}^{1 / 2} & \widetilde{\mu}_{\partial}^{0}
\end{array}\right),\left(\begin{array}{cc}
\widetilde{q}_{0} r_{T} & -i \widetilde{q}_{1} \\
i \widetilde{q}_{1} & \widetilde{q}_{0}
\end{array}\right)\right\rangle=0 .
$$

On a $\left.\widetilde{v}_{k, T}\right|_{\partial M}=-\left.(\operatorname{det} g)^{1 / 4} \nabla(\operatorname{det} g)^{-1 / 4} \widetilde{\varphi}_{k}\right|_{\partial M}$ où $\widetilde{\varphi}_{k}$ est une suite de solutions convergeant fortement vers 0 dans $H_{\varrho_{0}}^{2}$ (car $u_{k, L}$ tend vers 0 dans $H_{\varrho_{0}}^{1}$ ) de l'équation $\widetilde{P}_{L} \widetilde{\varphi}_{k}=\left(\partial_{x}^{2}+\widetilde{R}_{L}\right) \widetilde{\varphi}=0$ et ses traces $\left.\widetilde{\varphi}_{k}\right|_{\partial M}$ et $\left.\partial_{x} \widetilde{\varphi}_{k}\right|_{\partial M}$ sont bornées dans $H_{\varrho_{0}}^{2}$ et $H_{\varrho_{0}}^{1}$ respectivement. On a alors, si $Q=-Q_{2} \partial_{x}$ avec $Q_{2}$, un opérateur tangentiel d'ordre 2

$$
\int_{X}\left(P_{L}^{*} Q-Q P_{L}\right) \varphi_{k} \overline{\varphi_{k}}=\int_{\partial X} \partial_{x} Q_{2} \partial_{x} \varphi_{k} \overline{\varphi_{k}}+Q_{2} \partial_{x} \varphi_{k} \overline{\partial_{x} \varphi_{k}} .
$$

Le terme de gauche dans (5.48) tend vers 0 , celui de droite (modulo extraction d'une sous-suite) vers

$$
0=\left\langle\nu^{2}, r_{L} q_{2}\right\rangle+\left\langle\nu^{1}, q_{2}\right\rangle
$$

$4^{\text {e }}$ SÉRIE - TOME $34-2001-\mathrm{N}^{\circ} 6$ 
où $\nu_{2}$ et $\nu_{1}$ sont les mesures de défaut (voir P. Gérard [5] et L. Tartar [15]) dans $H^{2}(\partial M)$ et $H^{1}(\partial M)$ respectivement des suites $\left(\left.\widetilde{\varphi}_{k}\right|_{\partial M}\right)$ et $\left(\left.\partial_{x} \widetilde{\varphi}_{k}\right|_{\partial M}\right)$. Localisant cette dernière équation sur $\mathcal{G}_{L}$, on obtient $\nu^{1} 1_{\mathcal{G}_{L}}=0$. Comme $\left.\widetilde{v}_{k, T, x}\right|_{\partial M}=-\left.\partial_{x} \widetilde{\varphi}_{k}\right|_{\partial M}+\mathrm{o}(1)_{H^{1}}$, on en déduit que les mesures $\widetilde{\mu}_{\partial}^{1} 1_{\mathcal{G}_{L}}$ et $\widetilde{\mu}_{\partial}^{1 / 2} 1_{\mathcal{G}_{L}}$ sont de la forme

$$
\widetilde{\mu}_{\partial}^{1} 1_{\mathcal{G}_{L}}=1_{\mathcal{G}_{L}}\left(\begin{array}{ccc}
0 & 0 & 0 \\
0 & \widetilde{\mu}_{\partial, 2,2}^{1} & \widetilde{\mu}_{\partial, 2,3}^{1} \\
0 & \widetilde{\mu}_{\partial, 3,2}^{1} & \widetilde{\mu}_{\partial, 3,3}^{1}
\end{array}\right), \quad \widetilde{\mu}_{\partial}^{1 / 2} 1_{\mathcal{G}_{L}}=1_{\mathcal{G}_{L}}\left(\begin{array}{ccc}
0 & 0 & 0 \\
0 & \tilde{\mu}_{\partial, 2,2}^{1 / 2} & \tilde{\mu}_{\partial, 2,3}^{1 / 2} \\
0 & \tilde{\mu}_{\partial, 3,2}^{1 / 2} & \tilde{\mu}_{\partial, 3,3}^{1 / 2}
\end{array}\right) .
$$

Les relations

$$
\operatorname{div} \widetilde{v}_{k, T}=\partial_{x} \widetilde{v}_{k, T, x}+\partial_{y_{1}} \widetilde{v}_{k, T, y_{1}}+\partial_{y_{2}} \widetilde{v}_{k, T, y_{2}}=\mathcal{O}(1)_{H_{\varrho_{0}}^{1}}
$$

et

$$
\left.\widetilde{v}_{k, T, y}\right|_{\partial M}=-\nabla_{y} \widetilde{\varphi}+\mathrm{o}(1)_{H_{\varrho_{0}}^{1}}
$$

montrent

$$
\left.\widetilde{v}_{k, T, y}\right|_{\partial M}=C_{-1}\left(\frac{\partial_{x}}{i} \widetilde{v}_{k, T, x}\right)+\mathrm{o}(1)_{H_{\varrho_{0}}^{1}}
$$

où le symbole principal de l'opérateur $C_{-1}$ est $-\alpha(\eta) /\|\eta\|^{2}$.

Si on note

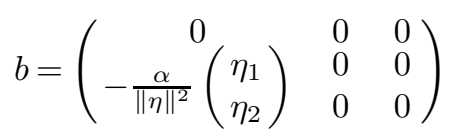

on peut réécrire le terme de gauche dans (5.47) sous la forme

$$
\left\langle 1_{\mathcal{G}_{L}} \widetilde{\mu}_{\partial}^{0}, \widetilde{q}_{0}+b^{*} \widetilde{q}_{0} r_{T} b+\widetilde{q}_{1} b+b^{*} \widetilde{q}_{1}\right\rangle
$$

et le même calcul algébrique qu'à la démonstration du lemme 2.10 montre que si $\widetilde{q}$ est pair, alors le terme de droite dans (5.55) est nul sur $\mathcal{G}_{L}$; ce qui termine la démonstration de la proposition 5.1 .

Terminons cette partie avec la définition suivante :

DÉFINITION 5.3. - On appellera rayon de polarisation transversale sur $\Omega$ toute application

$$
\gamma: s \in I \subset \mathbb{R} \mapsto \gamma(s) \in S \hat{Z} \times\left. T \mathbb{R}^{3}\right|_{\bar{\Omega}} \otimes_{\mathbb{R}} \mathbb{C}
$$

où $I$ est un intervalle de $\mathbb{R}, S \hat{Z}$ est l'espace associé à $M=\mathbb{R}_{t} \times \Omega$, défini en (2.36) et $\left.T \mathbb{R}^{3}\right|_{\Omega} \otimes_{\mathbb{R}} \mathbb{C}$ est le complexifié de l'espace tangent à $\bar{\Omega}$ et telle que :

1. La projection, $s \mapsto \pi_{x, t}(\gamma(s))$, sur la base de $S \hat{Z}, \mathbb{R}_{t} \times \bar{O}$ est un rayon bicaractéristique généralisé au sens de Melrose et Sjöstrand.

2. Si $\pi_{x, t}\left(\gamma\left(s_{0}\right)\right) \in \mathbb{R}_{t} \times \Omega$ alors la projection $s \mapsto \pi_{X}(\gamma(s))$ sur le dernier facteur est constante au voisinage de $s_{0}$ et orthogonale à la direction de propagation : $\left\langle\pi_{\xi}(\gamma(s)), \pi_{X}(\gamma(s))\right\rangle=0$.

3. Si $\pi_{x, t, \xi, \tau}\left(\gamma\left(s_{0}\right)\right) \in \mathcal{H}_{T} \cap \overline{\mathcal{H}_{L}}$ alors $\gamma\left(s_{0}-0\right)$ et $\gamma\left(s_{0}+0\right)$ existent et $s \mapsto \pi_{X}(\gamma(s))$ est continue près de $s_{0}$.

4. Si $\pi_{x, t, \xi, \tau}\left(\gamma\left(s_{0}\right)\right) \in \mathcal{E}_{L} \cap \mathcal{H}_{T}$ alors $\gamma\left(s_{0}-0\right)$ et $\gamma\left(s_{0}+0\right)$ existent et vérifient

$$
\pi_{X}\left(\gamma\left(s_{0}+0\right)\right)=J_{\pi_{\xi\left(\gamma\left(s_{0}-0\right)\right)}}\left(\pi_{X}\left(\gamma\left(s_{0}+0\right)\right)\right) .
$$


5. Si $\pi_{x, t, \xi, \tau}\left(\gamma\left(s_{0}\right)\right) \in \mathcal{E}_{T}$ alors $\pi_{X}(\gamma(s))$ est de classe $C^{1}$ près de $s_{0}$ et vérifie en $s_{0}$ l'équation différentielle (5.32) (sur les coordonnées du repère de Frénet).

C'est-à-dire que la variation de la composante du fibré tangent d'un rayon de polarisation est décrite par les différents cas que nous venons d'étudier dans cette section.

\section{Décroissance exponentielle de l'énergie pour les solutions du système de la thermoélasticité}

On considère $\Omega \subset \mathbb{R}^{d}, d=2,3$, un ouvert régulier, relativement compact, $\left(u_{0}, u_{1}, \theta_{0}\right) \in$ $H_{0}^{1}(\Omega)^{d} \times L^{2}(\Omega)^{d+1},(u, \theta)$ la solution donnée par le théorème de Hille Yosida du système

$$
\left\{\begin{array}{l}
\left(\partial_{t}^{2}-\mu \Delta-(\lambda+\mu) \nabla \operatorname{div}\right) u+\alpha \nabla \theta=0, \\
\partial_{t} \theta-\Delta \theta+\beta \operatorname{div} \partial_{t} u=0, \\
\left.u\right|_{\partial \Omega}=0,\left.\quad \theta\right|_{\partial \Omega}=0, \\
\left.u\right|_{t=0}=u_{0} \in H_{0}^{1}(\Omega)^{3},\left.\quad \partial_{t} u\right|_{t=0}=u_{1} \in L^{2}(\Omega)^{3}, \\
\left.\theta\right|_{t=0}=\theta_{0} \in L^{2}(\Omega) ;
\end{array}\right.
$$

et son énergie naturelle :

$$
E(u, \theta)(t)=\frac{1}{2} \int_{\Omega}\left[\left|\partial_{t} u\right|^{2}+\mu|\nabla u|^{2}+(\lambda+\mu)|\operatorname{div} u|^{2}+\frac{\alpha}{\beta}|\theta|^{2}\right] \mathrm{d} x,
$$

qui vérifie

$$
\frac{\mathrm{d} E}{\mathrm{~d} t}=-\frac{\alpha}{\beta} \int_{\Omega}|\nabla \theta|^{2} \mathrm{~d} x \leqslant 0 .
$$

Pour simplifier la présentation on se placera dans cette partie dans le cas le plus difficile à étudier (et pertinent du point de vue physique) où $\lambda+\mu>0$.

G. Lebeau et E. Zuazua ont démontré dans [11], théorème 1.1 (voir aussi relations (3.6) et (3.7)), le résultat suivant

THÉORÈME 2 (Lebeau-Zuazua).-La décroissance de l'énergie pour les solutions du système (6.1) est uniforme : il existe $C, \varepsilon>0$ tels que pour tous $\left(u_{0}, u_{1}, \theta_{0}\right) \in H_{0}^{1}(\Omega)^{d} \times$ $L^{2}(\Omega)^{d+1}$,

$$
E(u, \theta)(t) \leqslant C \mathrm{e}^{-\varepsilon t} E(u, \theta)(0),
$$

si et seulement si les deux conditions suivantes sont vérifiées :

i) Toute solution, $\varphi \in H_{0}^{1}(\Omega)^{d} d u$ système

$$
\begin{aligned}
& -\Delta \varphi=\gamma^{2} \varphi \quad \text { dans } \Omega, \\
& \operatorname{div} \varphi=0
\end{aligned}
$$

est nulle.

ii) Il existe $T>0$ et $C>0$ tels que pour tous $\left(u_{0}, u_{1}\right) \in H_{0}^{1}(\Omega)^{d} \times L^{2}(\Omega)^{d}$, la solution $d u$ système de Lamé (4.1) vérifie

$$
\left\|u_{0}\right\|_{H^{1}(\Omega)^{d}}^{2}+\left\|u_{1}\right\|_{L^{2}(\Omega)^{d}}^{2} \leqslant C \int_{0}^{T} \int_{\Omega}|\operatorname{div} u|^{2} \mathrm{~d} x \mathrm{~d} t .
$$


Remarque 6.1. - En toute rigueur, le théorème 1.1 de [11] correspond à prendre les normes $L^{2}$ et $H^{-1}$ dans le terme de gauche et la norme $H^{-1}$ dans le terme de droite de (6.7). Néanmoins l'opérateur $\sqrt{A_{D}}$ réalisant une isométrie de $H^{1}$ sur $L^{2}$ et de $L^{2}$ sur $H^{-1}$ et commutant à l'évolution, on voit facilement que l'équation (9) de [11] est bien équivalente à (6.7).

Dans la cas où $d=2$, ils en ont déduit une condition nécessaire et suffisante pour que (6.4) soit vérifiée. Nous allons généraliser cette étude au cas $d=3$.

THÉORÈME 3 (condition suffisante). - On suppose que $\Omega \subset \mathbb{R}^{3}$ n'a pas de contact d'ordre infini avec ses tangentes et que la condition i) précédente est vérifiée (c'est le cas génériquement dans la classe des ouverts $\left.C^{\infty}\right)$ et

iii) Il existe $T>0$ tel que tout rayon bicaractéristique généralisé pour l'opérateur $P_{T}=\nu_{T}^{2} \partial_{t}^{2}-\Delta$ rencontre l'ensemble

$$
\mathcal{H}_{L} \backslash\{\|\eta\|=0\} \cap\{t \in] 0, T[\}
$$

(au moins) deux fois et en deux points où les directions critiques qui sont en ces points les normales à la direction de propagation et à la normale au bord n'appartiennent pas au même rayon de polarisation transversale (voir définition 5.3 ).

Alors (6.4) est vérifiée (l'énergie décroît exponentiellement).

Remarque 6.2. - Si la condition iii) est vérifiée, alors la condition i) ne peut être violée que par un nombre fini de fonctions $\varphi$ et si on note $F$ l'espace vectoriel engendré par les triplets de la forme $u_{0}=c_{0} \varphi, u_{1}=c_{1} \varphi, \theta_{0}=0$, on obtiendrait sous l'hypothèse iii) que la distance de $\left(u(t), \partial_{t} u(t), \theta(t)\right)$ à $F$ décroît exponentiellement (voir [11], Remark 2.2).

THÉORÈME 4 (condition nécessaire).- Soit $T>0$. On suppose qu'il existe un rayon bicaractéristique généralisé pour l'opérateur $P_{T}=\nu_{T}^{2} \partial_{t}^{2}-\Delta$ qui ne rencontre l'ensemble

$$
\mathcal{H}_{L} \backslash\{\|\eta\|=0\} \cap\{t \in[0, T]\}
$$

qu'en des points où les directions critiques appartiennent au même rayon de polarisation transversale. Alors il existe une suite de données initiales $\left(u_{0}^{k}, u_{1}^{k}, \theta_{0}^{k}=0\right)$ telles que la solution de (6.1) vérifie

$$
\begin{aligned}
E\left(u_{0}^{k}, u_{1}^{k}, \theta_{0}^{k}, t=0\right) & =1, \\
\lim _{k \rightarrow+\infty} E\left(u_{0}^{k}, u_{1}^{k}, \theta_{0}^{k}, t=T\right) & =1 .
\end{aligned}
$$

Démonstration du théorème 3.-Un argument d'unicité-compacité désormais classique (voir [1]) permet de montrer que les conditions i) et ii) sont équivalentes aux conditions i) et ii' $^{\prime}$, avec

ii $^{\prime}$ ) Il existe $T>0$ et $C>0$ tels que pour tous $\left(u_{0}, u_{1}\right) \in H_{0}^{1}(\Omega)^{d} \times L^{2}(\Omega)^{d}$, la solution du système de Lamé (4.1) vérifie

$$
\begin{aligned}
& \left\|u_{0}\right\|_{H^{1}(\Omega)^{d}}^{2}+\left\|u_{1}\right\|_{L^{2}(\Omega)^{d}}^{2} \\
& \quad \leqslant C\left[\int_{0}^{T} \int_{\Omega}|\operatorname{div} u|^{2} \mathrm{~d} x \mathrm{~d} t+\left\|u_{0}\right\|_{L^{2}(\Omega)^{d}}^{2}+\left\|u_{1}\right\|_{H^{-1}(\Omega)^{d}}^{2}\right] .
\end{aligned}
$$

Nous allons montrer que la condition iii) implique la condition $\mathrm{ii}^{\prime}$ ). En effet supposons la condition iii) vérifiée et raisonnons par l'absurde. Si la condition ii') n'est pas vérifiée, alors on peut construire une suite $\left(u^{k}\right)$ de solutions du système de Lamé (4.1) vérifiant 


$$
\begin{aligned}
& \left\|u_{0}^{k}\right\|_{H^{1}(\Omega)^{d}}^{2}+\left\|u_{1}^{k}\right\|_{L^{2}(\Omega)^{d}}^{2} \\
& \quad>k\left[\int_{0}^{T} \int_{\Omega}\left|\operatorname{div} u^{k}\right|^{2} \mathrm{~d} x \mathrm{~d} t+\left\|u_{0}^{k}\right\|_{L^{2}(\Omega)^{d}}^{2}+\left\|u_{1}^{k}\right\|_{H^{-1}(\Omega)^{d}}^{2}\right] .
\end{aligned}
$$

Quitte à normaliser la suite $\left(u^{k}\right)$ et à extraire une sous-suite, on peut supposer que le terme de gauche est égal à 1 , et que la suite converge faiblement vers une limite qui d'après (6.13) ne peut être que 0 . On obtient ainsi une suite de solutions $\left(u^{k}\right)$ du système de Lamé convergeant faiblement vers 0 et vérifiant $\operatorname{div} u^{k} \rightarrow 0$ dans $L^{2}(] 0, T[\times \Omega)$. On peut alors appliquer à cette suite les constructions de la section précédente et on obtient ainsi une mesure $\mu_{T}$ (définie sur $S Z \cap] 0, T\left[_{t}\right.$ et invariante sur cet ensemble par le flot que nous avons décrit). On sait aussi que cette mesure est polarisée perpendiculairement à la direction de propagation et que près de tout point de $\mathcal{H}_{L} \backslash\{\|\eta\|=0\}$, elle est polarisée le long de la direction critique. Comme par hypothèse, tout rayon rencontre $] 0, T$ [ en au moins deux points qui ne sont pas connectés par la propagation, le théorème d'invariance de la mesure implique qu'en tout point de $] 0, T[$, la mesure est polarisée à la fois le long de 2 vecteurs linéairement indépendants (inclus dans le plan orthogonal à la direction de propagation), donc qu'elle est nulle. Comme

$$
\mu_{T}(S Z \cap] 0, T[)=T \times \lim _{k \rightarrow+\infty}\left\|u_{0}^{k}\right\|_{H^{1}(\Omega)^{d}}^{2}+\left\|u_{1}^{k}\right\|_{L^{2}(\Omega)^{d}}^{2}=1
$$

on obtient une contradiction.

Démonstration du théorème 4. - Notons $\gamma(s)$ un rayon bicaractéristique généralisé pour l'opérateur $P_{T}=\nu_{T}^{2} \partial_{t}^{2}-\Delta$ qui ne rencontre l'ensemble

$$
\mathcal{H}_{L} \backslash\{\|\eta\|=0\} \cap\{t \in[0, T]\}
$$

qu'en des points où les directions critiques sont connectées par la propagation. On peut supposer que sur $\gamma$, on à $\tau=+c_{T}$. On remarque d'abord que si $\gamma$ n'a pas de point intérieur dans $[0, T]$ $\left(\gamma \subset S^{*} \partial M\right)$, alors il est inclus dans la région $\mathcal{G}_{T} \subset \mathcal{E}_{L}$, donc si $\gamma_{n}$ est une suite de rayons réfléchis qui convergent vers $\gamma$ (il en existe puisque $\Omega$ n'a pas avec ses tangentes de contact d'ordre infini, voir [13]), pour $n$ assez grand, $\gamma_{n}$ ne peut rencontrer $S^{*} \partial M$ qu'en des points de la région $\mathcal{E}_{L}$, donc vérifie encore la même hypothèse. On peut donc supposer que $\gamma$ possède un point intérieur, $x_{0}$. On peut aussi supposer que ce point correspond sur $\gamma$ au temps $t=0$ et au paramètre $s=0$. On note $D=\left(D_{1}, D_{2}, D_{3}\right)$ la direction de polarisation obtenue pour $s=0$ et que le flot envoie sur les directions critiques obtenues à chaque contact du rayon avec $\mathcal{H}_{L} \backslash\{\|\eta\|=0\} \cap\{t \in[0, T]\}$. Par changement de variables orthonormales, on se ramène au cas $x_{0}=0, \xi_{0}=(1,0,0), \tau_{0}=c_{T}, t_{0}=0$ et $D=(0,1,0)$. On peut alors construire une suite de données initiales, $\left(u_{0}^{k}, u_{1}^{k}\right)$ d'énergies égales à 1 et telles que les mesures $\mu_{T, L}$ associées vérifient pour $|t| \ll 1$ :

a) $\mu_{L}=0$.

b) Le support de $\mu_{T}$ est $\gamma$ et de plus $\mu_{T}$ est polarisée le long de $D$.

En effet, pour $|t| \ll 1$, puisque le point $x_{0}$ est un point intérieur, la vitesse finie de propagation du support permet de ne pas tenir compte du bord, si on prend les données initiales à support assez petits. Soit $\psi \in C_{0}^{\infty}\left(\mathbb{R}^{3}\right)$. On considère les fonctions

$$
\psi_{k}(x)=C^{-1} k^{-5 / 4} \mathrm{e}^{i k x_{1}} \psi(\sqrt{k} x) ; \quad C=c_{T}\left(\int|\psi|^{2}\right)^{1 / 2}
$$


et

$$
u_{0}^{k}=\operatorname{rot}\left(\begin{array}{c}
0 \\
0 \\
-\psi^{k}
\end{array}\right)=\left(\begin{array}{c}
-\partial_{x_{2}} \psi^{k} \\
\partial_{x_{1}} \psi^{k} \\
0
\end{array}\right) ; \quad u_{1}^{k}=i k c_{T} u_{0}^{k}
$$

et on leur associe la suite $\left(u^{k}\right)$ de solutions du système de Lamé de données initiales $\left(u_{0}^{k}, u_{1}^{k}\right)$. L'énergie de la suite $\left(u^{k}\right)$ tend vers 1 et il est facile de voir que pour $|t| \ll 1$, d'une part la mesure $\mu_{L}$ est nulle et d'autre part la mesure $\mu_{T}$ est supportée par $\gamma$ et polarisée le long de $D=(0,1,0)$. Notons $D(s)$ la direction obtenue par propagation le long de $\gamma(s)$ de la direction $D, \mathcal{P}(t)$ la proposition :

$$
\left\{\begin{array}{l}
\text { «Au voisinage de }[0, t] \text { la mesure } \mu_{L} \text { est nulle et } \mu_{T} \text { est supportée } \\
\gamma \text { et polarisée le long de } D(s) . »
\end{array}\right.
$$

et $\mathcal{T}$ l'ensemble des $t \in[0, T]$ tels que $\mathcal{P}(t)$ est vérifiée. Cet ensemble est non vide et ouvert dans $[0, T]$. Si nous montrons qu'il est aussi fermé, on aura montré que la suite $u^{k}$ vérifie

$$
E\left(u^{k}\right) \rightarrow 1, \quad \text { et } \quad \operatorname{div} u^{k} \rightarrow 0 \text { dans } L^{2}(\Omega \times[0, T]) .
$$

Cette suite contredira donc l'estimation (6.7). On pourrait également en utilisant le résultat de découplage pour le système de la thermoélasticité de Lebeau-Zuazua [11], lemme 3.1, montrer que la suite de solutions du système de la thermoélasticité (6.1) associées à la suite de données initiales $\left(u_{0}^{k}, u_{1}^{k}, \theta_{0}=0\right)$ vérifie

$$
E\left(u^{k}, \theta^{k}\right)(0) \rightarrow 1, \quad \text { et } \quad E\left(u^{k}, \theta^{k}\right)(T) \rightarrow 1 .
$$

Montrons que l'ensemble $\mathcal{T}$ est fermé dans $[0, T]$. Supposons que $t_{n} \in \mathcal{T} \rightarrow t$. Le seul cas à étudier est $t_{n}<t, \forall n$. Soit $s_{0}$ le paramètre sur $\gamma$ correspondant à $t\left(s_{0}\right)=t$. Nous allons étudier les différents cas selon la nature du point $\varrho_{0}=\gamma\left(s_{0}\right)$. Il nous suffit de montrer que la mesure $\mu_{L}$ est nulle près de $\varrho_{0}$, et d'appliquer les résultats de la section précédente.

i) $\varrho_{0}$ est un point intérieur ;

ii) $\varrho_{0}$ est un point elliptique pour l'onde longitudinale ;

iii) $\varrho_{0}$ est un point hyperbolique pour l'onde longitudinale et $\|\eta\| \neq 0$;

iv) $\varrho_{0}$ est un point hyperbolique pour l'onde longitudinale et $\|\eta\|=0$;

v) $\varrho_{0}$ est un point diffractif ou glissant pour l'onde longitudinale.

\subsubsection{Les cas i) et ii)}

Le théorème d'invariance de la mesure $\mu_{T}$ au voisinage de $\varrho_{0}$ est encore vrai dans ces cas, puisque la démonstration n'utilise pas et prouve (car l'énergie est conservée) le fait que $\mu_{L}=0$. La propriété $\mathcal{P}\left(t_{0}\right)$ est donc vérifiée.

\subsubsection{Le cas iii)}

On reprend les notations de la section précédente. Supposons seulement qu'une des demibicaractéristiques issues de $\varrho_{0}$ (dont on exclut le point $\varrho_{0}$ ), par exemple celle correspondant à $\xi>0$, n'est pas dans le support de la mesure $\mu_{L}$. Par la théorie hyperbolique standard on sait alors qu'il existe un opérateur elliptique d'ordre $1, E_{-1}$, tel que près de $\rho_{0}$,

$$
\left.\widetilde{\varphi}\right|_{\partial M}=E_{-1}\left(\left.\frac{\partial \widetilde{\varphi}}{i \partial x}\right|_{\partial M}\right)+\mathrm{o}(1)_{H_{\partial, \rho_{0}}^{2}}
$$


et le symbole principal $e_{-1}$ de $E_{-1}$ vaut

$$
e_{-1}(y, \eta)=\frac{1}{\sqrt{\tau^{2} \nu_{L}^{2}-\|\eta\|^{2}}} .
$$

En reprenant l'étude réalisée à la section précédente près d'un point $\varrho_{0} \in \mathcal{E}_{L}$, on obtient que le système de conditions aux limites que $\widetilde{v}_{k, T}$ vérifie près de $\varrho_{0}$ est de la forme

$$
\widetilde{v}_{k, T}=B_{-1}^{\prime}\left(\frac{\partial \widetilde{v}_{k, T}}{i \partial x}\right)+\mathrm{o}(1)_{H_{\partial, \rho_{0}}^{1}},
$$

où $B_{-1}^{\prime}$ est une matrice $3 \times 3$ d'opérateurs pseudo-différentiels d'ordre -1 de symbole principal

$$
b_{-1}^{\prime}(y, \eta)=\left(\begin{array}{ccc}
\frac{-\sqrt{\tau^{2} / \nu_{L}^{2}-\|\eta\|^{2}}}{\|\eta\|^{2}} & 0 & 0 \\
-\frac{\alpha}{\|\eta\|^{2}}\left(\begin{array}{l}
\eta_{1} \\
\eta_{2}
\end{array}\right) & 0 & 0 \\
0
\end{array}\right) .
$$

Nous nous trouvons donc exactement dans le cadre d'application du théorème de propagation de la mesure de la section 3. Il ne reste donc qu'à identifier les endomorphismes $J_{\xi}$. Décrivons l'opérateur $J_{\xi}^{\prime}=T \chi J_{\chi} T_{\xi}^{-1}$. On a $J_{\xi}^{\prime}=\left(I d-\xi b_{-1}^{\prime}\right)^{-1}\left(I d+\xi b_{-1}^{\prime}\right)$, soit

$$
J_{\xi}^{\prime}=\left(\begin{array}{ccc}
\frac{\|\eta\|^{2}-\xi \delta}{\|\eta\|^{2}+\xi \delta} & 0 & 0 \\
\frac{-2 \xi}{\|\eta\|^{2}+\xi \delta} \alpha\left(\begin{array}{l}
\eta_{1} \\
\eta_{2}
\end{array}\right) & \left(\begin{array}{l}
1 \\
0
\end{array}\right) & \left(\begin{array}{l}
0 \\
1
\end{array}\right)
\end{array}\right),
$$

avec $\delta=\sqrt{\tau^{2} \nu_{L}^{2}-\|\eta\|^{2}}$. Compte tenu de ce que la mesure $\mu_{T}$ est polarisée perpendiculairement à la direction de propagation $(\xi, \eta)$, on ne s'intéresse encore qu'à son action sur le plan $(\xi, \eta)^{\perp}$. Une base de ce plan est donnée par les vecteurs

$$
e_{1}(\xi)=(0, Y), \quad e_{2}(\xi)=\left(\|\eta\|^{2}, \alpha(\eta) \xi\right),
$$

où $Y$ est un vecteur tangent au bord, de norme 1 et tel que $\langle Y, \eta\rangle=0$. On a alors

$$
\begin{aligned}
J_{\xi}^{\prime}\left(e_{1}(-\xi)\right) & =e_{1}(\xi), \\
J_{\xi}^{\prime}\left(e_{2}(-\xi)\right) & =\frac{\|\eta\|^{2}-\xi \delta}{\|\eta\|^{2}+\xi \delta} e_{2}(\xi) .
\end{aligned}
$$

Puisque l'onde incidente $\mu_{T}$ est polarisée le long de $D(s)$ et que $D\left(s_{0}\right)=e_{1}$, le calcul précédent montre que l'onde réfléchie est de même masse totale. La conservation de l'énergie pour les solutions du système de Lamé implique donc que $\mu_{L}$ est nulle au voisinage de $\varrho_{0}$. La propriété $\mathcal{P}\left(t_{0}\right)$ est donc vérifiée.

\subsubsection{Le cas iv)}

On ne fait pas dans ce cas d'hypothèse sur la polarisation de l'onde incidente. D'après l'étude précédente, on sait a priori que la mesure $\mu_{L}$ est supportée près de $\varrho_{0}$ par la réunion des rayons qui rencontrent l'ensemble $\eta=0$ (rayons normaux au bord). Donc la mesure $H^{2}$ de $\widetilde{\varphi}$ est supportée par ce même ensemble et celle de $\left.\widetilde{\varphi}\right|_{\partial M}$ aussi, donc

$$
\begin{aligned}
& \left.\widetilde{v}_{k, T, 1}\right|_{\partial M}=-\partial_{y_{1}} \widetilde{\varphi}+\mathrm{o}(1)_{H_{\varrho_{0}}^{1}}=\mathrm{o}(1)_{H_{\varrho_{0}}^{1}}, \\
& \left.\widetilde{v}_{k, T, 2}\right|_{\partial M}=-\partial_{y_{2}} \widetilde{\varphi}+\mathrm{o}(1)_{H_{\varrho_{0}}^{1}}=\mathrm{o}(1)_{H_{\varrho_{0}}^{1}},
\end{aligned}
$$


puisque les symboles $\eta_{1,2}$ s'annulent sur l'ensemble $\{\eta=0\}$. On obtient donc d'après l'équation (5.9)

$$
\left.\partial_{x} v_{k, T, x}\right|_{\partial M}=\mathrm{o}(1)_{L_{\varrho_{0}}^{2}} .
$$

Il y a donc réflexion totale pour la mesure $\mu_{T}$ et le même argument de conservation de l'énergie implique que $\mu_{L}=0$ près de $\varrho_{0}$. La propriété $\mathcal{P}\left(t_{0}\right)$ est donc aussi vérifiée.

\subsubsection{Le cas $v$ )}

L'étude de ce cas est proche de celle réalisée pour l'étude de la transmission à travers une interface dans une géométrie similaire à la nôtre par L. Miller [12]. On note $\mu$ la mesure associée à $\partial_{t} \widetilde{\varphi}$ et

$$
\left(\begin{array}{cc}
\mu_{\partial}^{1} & \mu_{\partial}^{*, 1 / 2} \\
\mu_{\partial}^{1 / 2} & \mu_{\partial}^{0}
\end{array}\right)
$$

les mesures de ses traces sur le bord (qui sont bornées dans $H^{1}$ et $L^{2}$ respectivement puisque $\varrho_{0} \in \mathcal{H}_{T}$ ). Soit $Q=Q_{1}+Q_{0} \frac{\partial_{x}}{i}$, avec $Q_{i}$ des opérateurs pseudodifférentiels tangentiels d'ordre $i$ à support compact.

En calculant $\left(\left[\widetilde{P}_{L}, Q\right] \partial_{t} \widetilde{\varphi}, \partial_{t} \widetilde{\varphi}\right)$ et en intégrant par parties, on obtient

$$
\begin{aligned}
& \left\langle\mu, \frac{-2 r_{L} \partial_{x} q_{0}-2 \xi \partial_{x} q_{1}+\left\{r_{L}, q_{0} \xi+q_{1}\right\}}{\lambda^{2}}\right\rangle \\
& =\left\langle\mu_{\partial}^{1}, \frac{q_{0} r_{L}}{|\eta|^{2}}\right\rangle+\left\langle\mu_{\partial}^{0}, q_{0}\right\rangle+2\left\langle\operatorname{Re} \mu_{\partial}^{1 / 2}, \frac{q_{1}}{|\eta|}\right\rangle .
\end{aligned}
$$

On prend dans un premier temps $q_{1}=0$ et

$$
q_{0}(x, y, t, \eta, \tau)=q_{0}^{0}(y, t, \eta, \tau) \theta(x / \varepsilon) \psi\left(r_{L} / \varepsilon^{2}\right),
$$

avec $\theta$ et $\psi$ deux fonctions $C^{\infty}$ à supports compacts égales à 1 au voisinage de 0 . On obtient par convergence dominée

$$
\left\langle\mu, \frac{-\partial_{x} r_{L} q_{0}}{\lambda^{2}} 1_{x=0} 1_{\mathcal{G}_{L}}\right\rangle=\left\langle\mu_{\partial}^{0}, q_{0}^{0} 1_{\mathcal{G}_{L}}\right\rangle .
$$

Soit $\varrho_{1}$ un point du bord voisin mais différent de $\varrho_{0}$. Comme la mesure $\mu_{T}$ incidente est nulle au voisinage de $\varrho_{1}$, on obtient d'après la théorie hyperbolique standard, près de $\varrho_{1}$, une relation pseudodifférentielle sur les deux traces de $\widetilde{v}_{k}$ de la forme

$$
\left(\widetilde{v}_{k}\right)_{\partial M}=E_{-1}\left(\partial_{x}\left(\widetilde{v}_{k}\right)_{\partial M}\right)+o(1)_{H_{\varrho_{1}}^{1}}
$$

où $E_{-1}$ est un o.p.d. scalaire d'ordre -1 de symbole principal

$$
e_{-1}(y, \eta)=\frac{1}{\sqrt{\tau^{2} \nu_{T}^{2}-\|\eta\|^{2}}}
$$

D'après (5.8) et (5.9), on obtient pour $\partial_{t} \widetilde{\varphi}$ la condition aux limites

$$
\partial_{t} \widetilde{\varphi}_{\partial M}=B_{-1}\left(\frac{\partial_{x}}{i} \partial_{t} \widetilde{\varphi}_{\partial M}\right)+\mathrm{o}(1)_{H_{\varrho_{1}}^{1}}
$$


où $B_{-1}$ est un o.p.d. d'ordre -1 de symbole principal

$$
b_{-1}(y, \eta)=\frac{\sqrt{\tau^{2} / \nu_{T}^{2}-\|\eta\|^{2}}}{\|\eta\|^{2}} .
$$

Nous pouvons donc appliquer le théorème de propagation pour la mesure $\mu$ qui est donc invariante près de $\varrho_{1}$ par le flot associé à cette condition aux limites. Il en résulte que si $s \mapsto \gamma(s)$ est la bicaractéristique généralisée de $-\xi^{2}+r_{L}$ vérifiant $\gamma(0)=\varrho_{0}$ (avec $s \mapsto t(s)$ croissant), on a

$$
\left\{\begin{array}{l}
\text { Le support de } \mu \text { est contenu dans }\{\gamma(s) ; s \geqslant 0\} \\
\text { et } \mu \text { est invariante par le flot précédent } \operatorname{sur}\{\gamma(s) ; s>0\} .
\end{array}\right.
$$

Comme près de $\varrho_{0}, \gamma(s)$ ne rencontre pas $\mathcal{H}_{L}$, le crochet de Poisson $\left\{-\xi^{2}+r_{L}, q_{1}\right\}(\gamma(s))$ vaut $\tau \frac{\mathrm{d}}{\mathrm{d} s} q_{1}(\gamma(s))$ (voir (3.29)). Il résulte alors de (6.33)

$$
\left\langle\mu, \frac{\tau}{\lambda^{2}} \frac{\mathrm{d}}{\mathrm{d} s} q_{1}(\gamma(s))\right\rangle=2\left\langle\operatorname{Re} \mu_{\partial}^{1 / 2}, \frac{q_{1}}{|\eta|}\right\rangle .
$$

Or les bicaractéristiques peuvent être paramétrées par une des variables tangentes $(t, y, \tau, \eta)$, donc (6.41) implique que $\frac{\mathrm{d} \mu}{\mathrm{d} s}$ est une mesure. En particulier $\mu$ ne charge pas $\varrho_{0}$. D'après (6.35), $\mu_{\partial}^{0}$ ne charge donc pas non plus $\varrho_{0}$. L'inégalité de Cauchy-Schwartz implique

$$
\left|\mu_{\partial}^{1 / 2}\right| \leqslant C \sqrt{\left|\mu_{\partial}^{0}\right|\left|\mu_{\partial}^{1}\right|} .
$$

La mesure $\mu_{\partial}^{1 / 2}$ ne charge donc pas non plus $\varrho_{0}$; donc (6.41) implique que $\frac{\mathrm{d} \mu}{\mathrm{d} s}$ est une mesure qui ne charge pas $\varrho_{0}$. D'après (6.40) et la formule des sauts, la mesure $\mu$ est donc nulle; ce qui implique $u_{k, L} \rightarrow 0$ dans $H_{\varrho_{0}}^{1}$. On peut donc appliquer le résultat de la section précédente qui démontre que la propriété $\mathcal{P}\left(t_{0}\right)$ est vérifiée.

\section{Appendice A. Les théories elliptique et hyperbolique «standard»}

Dans cette annexe nous démontrons les résultats classiques près des points hyperboliques ou elliptiques. Nous démontrons ces résultats dans le cas elliptique par la technique des projecteurs de Calderón et dans le cas hyperbolique par des estimations d'énergie.

\section{A.1. La théorie elliptique}

Nous montrons dans cette partie deux résultats : d'une part qu'indépendamment de toute condition aux limites les deux traces de la solution d'un problème elliptique d'ordre 2 sont liées par une relation pseudodifférentielle et que dans le cas d'un problème aux limites vérifiant les conditions de Lopatinski, ces deux traces sont essentiellement nulles dans la région elliptique. On limitera notre étude à celle d'un problème aux limites et nous supposerons connus les résultats dans le cas intérieur.

\section{A.1.1. Sans condition aux limites}

On reprend les notations de la section 2 et on se place près d'un point $\varrho_{0}=\left(x_{0}=0, y_{0}, \eta_{0}\right) \in \mathcal{E}$, ce qui se traduit par la relation

$$
-r(x, y, \eta) \geqslant C\|\eta\|^{2}
$$

$4^{\text {e }}$ SÉRIE - TOME $34-2001-\mathrm{N}^{\circ} 6$ 
près de $\varrho_{0}$. On considère une suite $\left(u^{k}\right)$ bornée et convergeant faiblement vers 0 dans $H^{1}(] 0,1[\times Y)$ et telle que

$$
\begin{aligned}
P u^{k} & =\left(\left(\partial_{x}^{2}+R\left(x, y, D_{y}\right)\right) \mathrm{Id}+M_{0} \partial_{x}+M_{1}\right) u^{k} \\
& =v^{k} \rightarrow 0 \quad \text { dans } L^{2}(] 0,1[\times Y) .
\end{aligned}
$$

Soient $\varphi \in C_{0}^{\infty}\left(\mathbb{R}^{d+1}\right)$ à support proche de $\left(x_{0}, y_{0}\right)$ et égale à 1 près de ce point et $\psi \in C^{\infty}\left(\mathbb{R}^{d}\right)$ homogène de degré 0 pour $|\eta| \geqslant 1$ à support dans un petit voisinage conique de $\eta_{0}$ et égale à 1 près de ce point. On note $p(x, y, \xi, \eta)=\left(-\xi^{2}+r(x, y, \eta)\right)$ Id et $m(x, y, \xi, \eta)$ un symbole matriciel (polynomial en la variable $\xi$ ) tel que

$$
P=\mathrm{Op}\left(\left(-\xi^{2}+r(x, y, \eta)\right) \mathrm{Id}+m(x, \xi, y, \eta)\right) .
$$

On définit

$$
E_{M}=\sum_{l=0}^{M} E^{l}
$$

une paramétrixe de l'opérateur $P$ à l'ordre $M$ par la procédure suivante :

$$
E^{0}=\operatorname{Op}\left(\frac{\varphi(x, y) \psi(\eta)}{-\xi^{2}+r(x, y, \eta)}\right) \mathrm{Id}
$$

et les $E^{l}=\left(E_{i, j}^{l}\right)_{i, j=1}^{N}$ sont des matrices $N \times N$ d'opérateurs pseudodifférentiels définis par récurrence par les relations

$$
E^{1} \times p=-\sum_{|\alpha|=1} \frac{1}{i^{|\alpha|}} \partial_{\xi, \eta}^{\alpha} E^{0} \times \partial_{x}^{\alpha} p-E^{0} \times m
$$

$$
E^{n} \times p=-\sum_{\substack{|\alpha|+k=n, k \neq n,}} \frac{1}{i^{|\alpha|}} \partial_{\xi, \eta}^{\alpha} E^{k} \times \partial_{x}^{\alpha} p-\sum_{|\alpha|+k=n-1} \frac{1}{i^{|\alpha|}} \partial_{\xi, \eta}^{\alpha} E^{k} \times \partial_{x}^{\alpha} m,
$$

où $p=\left(-\xi^{2}+r\right)$ Id et $\times$ désigne la multiplication des matrices (si on a choisi les supports des fonctions $\varphi$ et $\psi$ assez petits, $p$ est inversible sur le support des termes de droite de (A.6), (A.7)).

Le calcul symbolique des opérateurs pseudodifférentiels montre que

$$
E_{M} \circ P=\varphi(x) \psi\left(D_{y}\right) \mathrm{Id}+R_{M},
$$

où $R_{M}$ est un opérateur pseudodifférentiel d'ordre $-1-M$.

On note $\underline{u^{k}}$ le prolongement par 0 dans $\{x<0\}$ de $u^{k}$. D'après la formule des sauts, on a

$$
P \underline{u^{k}}=\left.\partial_{x} u^{k}\right|_{x=0} \otimes \delta_{x=0}-\left.u\right|_{x=0} \otimes \delta_{x=0}^{\prime}+\left.M_{0}\left(0, y, D_{y}\right) u\right|_{x=0} \otimes \delta_{x=0}+\underline{v^{k}} .
$$

Soit $\tilde{\varphi} \in C_{0}^{\infty}\left(\mathbb{R}^{1+d}\right)$, égale à 1 au voisinage du support de $\varphi$. Appliquant $E_{M} \circ \tilde{\varphi}$ à (A.9) (avec $M \geqslant 3$ ), on obtient

$$
\begin{aligned}
\varphi(x, y) \psi\left(D_{y}\right) \underline{u^{k}}= & E_{M}\left(\tilde{\varphi}(x=0, y)\left(\left.\partial_{x} u^{k}\right|_{x=0}+\left.M_{0}\left(0, y, D_{y}\right) u\right|_{x=0}\right) \otimes \delta_{x=0}\right. \\
& \left.-\left.\tilde{\varphi}(x=0, y) u\right|_{x=0} \otimes \delta_{x=0}^{\prime}\right)+w^{k}
\end{aligned}
$$


avec

$$
\begin{aligned}
& w^{k} \rightarrow 0 \quad \text { dans } H^{2}(]-1,1[\times Y), \\
& \partial_{x} w^{k} \rightarrow 0 \quad \text { dans } H^{2}(]-1,1\left[; H^{-1}(Y)\right) .
\end{aligned}
$$

Notons $e_{M}(x, y, \xi, \eta)$ le symbole de l'opérateur $E_{M}$ qui est d'après (A.5), (A.6) et (A.7) méromorphe en la variable $\xi$ avec des pôles aux points $\xi^{ \pm}= \pm i \sqrt{-r(x, y, \eta)}$. On a

$$
\begin{gathered}
E_{M}\left(\tilde{\varphi}(x=0, y)\left(\left.\partial_{x} u^{k}\right|_{x=0}+\left.M_{0}\left(0, y, D_{y}\right) u\right|_{x=0}\right) \otimes \delta_{x=0}\right)(x, y) \\
=(2 \pi)^{-d-1} \int \mathrm{e}^{i\left(x \cdot \xi+\left(y-y^{\prime}\right) \cdot \eta\right)} e_{M}(x, y, \xi, \eta) \tilde{\varphi}(x=0, y) \\
\quad \times\left(\left.\partial_{x} u^{k}\right|_{x=0}+\left.M_{0}\left(0, y, D_{y}\right) u\right|_{x=0}\right)\left(y^{\prime}\right) \mathrm{d} y^{\prime} \mathrm{d} \eta \mathrm{d} \xi .
\end{gathered}
$$

Pour calculer cette expression pour $x<0$, on déforme le contour d'intégration en $\xi$ dans le demi-plan $\{\operatorname{Im} \xi<0\}$ et on obtient par la formule des résidus (pour $x<0$ )

$$
\begin{aligned}
& E_{M}\left(\left(\left.\partial_{x} u^{k}\right|_{x=0}+\left.M_{0}\left(0, y, D_{y}\right) u\right|_{x=0}\right) \otimes \delta_{x=0}\right)(x, y) \\
&=(2 \pi)^{-d} \mathrm{e}^{i \xi^{-}(x, y, \eta) \cdot x} \int \mathrm{e}^{i\left(y-y^{\prime}\right) \cdot \eta} n_{M}(x, y, \eta) \tilde{\varphi}(x=0, y) \\
& \quad \times\left(\left.\partial_{x} u^{k}\right|_{x=0}+\left.M_{0}\left(0, y, D_{y}\right) u\right|_{x=0}\right) \mathrm{d} y^{\prime} \mathrm{d} \eta,
\end{aligned}
$$

où $n_{M}(x, y, \eta)$ est le résidu de la fonction $-i e_{M}$ au point $\xi^{-}$. En particulier $n_{M}$ est un symbole de degré -1 de partie principale égale à

$$
\sigma_{-1}\left(n_{M}\right)=\frac{-\varphi(x, y) \psi(\eta)}{2 \sqrt{-r(x, y, \eta)}} .
$$

De la même façon on calcule pour $x<0$

$$
\begin{aligned}
& E_{M}\left(-\left.\tilde{\varphi}(x=0, y) u^{k}\right|_{x=0} \otimes \delta_{x=0}^{\prime}\right)(x, y) \\
& \quad=\left.(2 \pi)^{-d} \mathrm{e}^{i \xi^{-}(x, y, \eta) \cdot x} \int \mathrm{e}^{i\left(y-y^{\prime}\right) \cdot \eta} d_{M}(x, y, \eta) \tilde{\varphi}(x=0, y) u\right|_{x=0}\left(y^{\prime}\right) \mathrm{d} y^{\prime} \mathrm{d} \eta,
\end{aligned}
$$

où $d_{M}(x, y, \eta)$ est un symbole de degré 0 de partie principale égale à

$$
\sigma_{0}\left(d_{M}\right)=\frac{-\varphi(x, y) \psi(\eta)}{2}
$$

Puisque $\underline{u^{k}}=0$ dans l'ensemble $\{x<0\}$, on obtient d'après (A.10), (A.11) que la somme des termes apparaissant dans (A.13) et (A.15) tend vers 0 dans $H^{2}(]-1,0[\times Y)$, ce qui implique en prenant la trace sur $x=0^{-}$que

$$
\left.\mathrm{Op}\left(n_{M}\right)\right|_{x=0}\left(\left.\partial_{x} u^{k}\right|_{x=0}\right)-\left.\mathrm{Op}\left(d_{M}\right)\right|_{x=0}\left(\left.u^{k}\right|_{x=0}\right) \rightarrow 0 \quad \text { dans } H^{3 / 2}(Y) ;
$$

ce qui montre qu'indépendamment de toute condition aux limites il existe une relation pseudodifférentielle entre les deux traces.

\section{A.1.2. La condition aux limites}

On suppose que la suite $\left(u^{k}\right)$ vérifie de plus une condition aux limites (avec $A$ et $B$ des opérateurs pseudodifférentiels de degré 0 et 1 respectivement)

$$
A\left(\left.u\right|_{x=0}\right)+B\left(\left.\partial_{x} u\right|_{x=0}\right) \rightarrow 0 \quad \text { dans } H^{1}(Y) .
$$


On suppose que cette condition aux limites vérifie les conditions de Lopatinski uniformes, ce qui se traduit simplement par le fait que le système

$$
\left(\begin{array}{cc}
-\sigma_{-1}\left(d_{M}\right) & \sigma_{0}\left(n_{M}\right) \\
\sigma_{0}(A) & \sigma_{1}(B)
\end{array}\right)
$$

est indépendant.

On déduit alors de (A.17), (A.18)

$$
\begin{aligned}
& \left.u\right|_{x=0} \rightarrow 0 \quad \text { dans } H_{\varrho_{0}}^{1}, \\
& \left.\partial_{x} u\right|_{x=0} \rightarrow 0 \quad \text { dans } L_{\varrho_{0}}^{2} .
\end{aligned}
$$

\section{A.2. La théorie hyperbolique}

Dans cette partie nous démontrons trois résultats dans le cas microlocalement hyperbolique pour une suite de solutions bornée dans l'espace d'énergie $H^{1}$ : d'une part que les traces ont la régularité $H^{1}$ et $L^{2}$ respectivement, d'autre part que si on suppose que la solution est meilleure (à l'intérieur) sur une des deux demi-bicaractéristiques alors on a une relation pseudodifférentielle entre les deux traces; et enfin que si elle est meilleure que $H^{1}$ sur les deux demibicaractéristiques, alors les deux traces sont «nulles».

On se place près d'un point $\varrho_{0}$ hyperbolique, ce qui se traduit par

$$
r(x, y, \eta) \geqslant c|\eta|^{2}
$$

près de $\varrho_{0}$. On considère une suite $u^{k}$ bornée dans $H^{1}(] 0,1[\times Y)$ et telle que

$$
P u^{k}=\left(\left(\partial_{x}^{2}+R\left(x, y, D_{y}\right)\right) \operatorname{Id}+M_{0} \partial_{x}+M_{1}\right) u^{k}=v^{k} \rightarrow 0 \quad \text { dans } L^{2}(] 0,1[\times Y) .
$$

Les résultats de cette partie reposent sur le lemme de factorisation suivant (voir [8, lemme 23.2.8]) :

Lemme 6.1. - Soit $\varrho_{0} \in \mathcal{H}$ un point hyperbolique. Alors il existe $\Lambda^{ \pm}=\operatorname{Op}\left(\lambda^{ \pm}(x, y, \eta)\right)$ et $T=\mathrm{Op}(t(x, y, \eta))$ des opérateurs pseudodifférentiels tangentiels d'ordre 1 et $-\infty$ respectivement tels que près de $\varrho_{0}$ on a

$$
\begin{aligned}
P & =\partial_{x}^{2}+R\left(x, y, D_{y}\right)+M_{0}\left(x, y, D_{y}\right) \partial_{x}+M_{1}\left(x, y, D_{y}\right) \\
& =-\left(D_{x}-\Lambda^{+}\left(x, y, D_{y}\right)\right)\left(D_{x}-\Lambda^{-}\left(x, y, D_{y}\right)+T\left(x, y, D_{y}\right)\right) .
\end{aligned}
$$

De plus le symbole principal de $\Lambda^{ \pm}$est près de $\varrho_{0}$ égal à $\sigma_{1}\left(\Lambda^{ \pm}\right)=\sqrt{r}$.

Démonstration. - La preuve de ce lemme est identique à celle de Hörmander dans le cas scalaire. Nous la rappelons : on procède par approximations successives. On choisit d'abord $\Lambda_{1}^{+}$ un opérateur pseudodifférentiel de symbole principal égal près de $\varrho_{0}$ à $\lambda_{1}^{+}(x, y, \eta)=\sqrt{r}(x, y, \eta)$. On pose $\Lambda_{1}^{-}=-\Lambda_{1}^{+}+i M_{0}$ et on obtient

$$
\left(D_{x}-\Lambda_{1}^{+}\right)\left(D_{x}-\Lambda_{1}^{-}\right)=D_{x}^{2}-\left(\Lambda_{1}^{+}+\Lambda_{1}^{-}\right) D_{x}-\Lambda_{1}^{+} \lambda_{1}^{-}-\frac{1}{i} \partial_{x}\left(\Lambda_{1}^{-}\right) .
$$

Comme le symbole principal de $-\Lambda_{1}^{+} \Lambda_{1}^{-}$est le même que celui de $R$ on obtient que

$$
P+\left(D_{x}-\Lambda_{1}^{+}\right)\left(D_{x}-\Lambda_{1}^{-}\right)=T_{1},
$$


où $T_{1}$ est un opérateur pseudo-différentiel tangentiel de degré 1.

Supposons maintenant qu'on a construit $\Lambda_{i}^{ \pm}$et $T_{i}$ des opérateurs pseudodifférentiels de degrés respectifs 1 et $2-i$ tels que

$$
P+\left(D_{x}-\Lambda_{i}^{+}\right)\left(D_{x}-\Lambda_{i}^{-}\right)=T_{i}
$$

On cherche alors $\Lambda_{i+1}$ sous la forme $\Lambda_{i+1}^{ \pm}=\Lambda_{i}^{ \pm}+S_{i+1}^{ \pm}$où les $S_{i+1}^{ \pm}$sont des opérateurs pseudodifférentiels tangentiels de degré $2-(i+1)$. On obtient

$$
\begin{aligned}
P+\left(D_{x}-\Lambda_{i+1}^{+}\right)\left(D_{x}-\Lambda_{i+1}^{-}\right)= & T_{i}+S_{i+1}^{+}\left(D_{x}-\Lambda_{i}^{-}\right)+\left(D_{x}-\Lambda_{i}^{+}\right) S_{i+1}^{-}+S_{i+1}^{+} S_{i+1}^{-}, \\
= & T_{i}+\left(S_{i+1}^{+}+S_{i+1}^{-}\right) D_{x}-S_{i+1}^{+} \Lambda_{i}^{-}-S_{i+1}^{-} \Lambda_{i}^{+} \\
& +\left[D_{x}-\Lambda_{i}^{+}, S_{i+1}^{-}\right]+S_{i+1}^{+} S_{i+1}^{-} .
\end{aligned}
$$

Comme le symbole principal de $\Lambda_{i}^{ \pm}$est, d'après notre construction scalaire (et égal à $\pm \sqrt{r}$ ), la contribution de la dernière ligne dans (A.27) est de degré $2-(i+1)$. Si on prend

$$
S_{i+1}^{+}+S_{i+1}^{-}=0
$$

et $S_{i+1}^{+}$un opérateur pseudodifférentiel tangentiel de symbole principal égal à

$$
\sigma_{2-(i+1)}\left(S_{i+1}^{+}\right)=\frac{\sigma_{i}\left(T_{i}\right)}{-\sqrt{r}}
$$

on voit facilement que le terme de droite dans (A.27) est un opérateur pseudodifférentiel tangentiel de degré $2-(i+1)$. Une procédure de sommation des symboles à la Borel permet alors de définir $\Lambda_{+\infty}^{ \pm}$(pour notre propos, on aurait pu en fait se limiter a définir une factorisation approchée, c'est-à-dire à définir les $\Lambda_{i}^{ \pm}$pour $i$ assez grand).

Remarque 6.2. - On peut de la même façon construire des opérateurs $\widetilde{\Lambda}^{ \pm}$de symboles principaux égaux à $\pm \sqrt{r}$ et $\widetilde{T}$ régularisant tels que

$$
\begin{aligned}
P & =\partial_{x}^{2}+R\left(x, y, D_{y}\right)+M_{0}\left(x, y, D_{y}\right) \partial_{x}+M_{1}\left(x, y, D_{y}\right), \\
& =-\left(D_{x}-\widetilde{\Lambda}^{-}\left(x, y, D_{y}\right)\right)\left(D_{x}-\widetilde{\Lambda}^{+}\left(x, y, D_{y}\right)+\widetilde{T}\left(x, y, D_{y}\right)\right) .
\end{aligned}
$$

Soit $q_{0}(y, \eta)$ un symbole de degré 0 égal à 1 dans un voisinage conique de $\varrho_{0}$ et à support proche de $\varrho_{0}$. On note $q^{ \pm}(x, y, \eta)$ la solution de l'équation

$$
\left(\partial_{x} \mp H_{\sqrt{r}(x, y, \eta)}\right) q^{ \pm}=0,\left.\quad q^{ \pm}\right|_{x=0}=q_{0},
$$

où $H_{\sqrt{r}}$ est le champ hamiltonien associé à $\sqrt{r}$ (ici la variable $x$ est considérée comme un paramètre). Si le support de $q_{0}$ est assez proche de $\varrho_{0}$, cette équation a bien un sens (au moins pour $|x|$ assez petit). On remarquera aussi que $q^{ \pm}$est alors invariante le long de la projection en $(x, y, \eta), \gamma^{ \pm}$, de la courbe intégrale du champ hamiltonien du symbole $p=\xi^{2}-r(x, y, \eta)$ issue du point $\varrho=(x=0, y, \xi= \pm \sqrt{r}(0, y, \eta), \eta)$.

Soit $\varphi \in C_{0}^{\infty}\left(\mathbb{R}_{x}\right)$ égale à 1 au voisinage de 0 . Le symbole $\varphi(x) q(x, y, \eta)$ est alors à support compact en $x, y$ et ce support peut être choisi arbitrairement proche de $\gamma^{ \pm}$si le support de $q_{0}$ est choisi proche de $\varrho_{0}$. On notera $Q^{ \pm}=\operatorname{Op}\left(q^{ \pm}\right)$et

$$
w_{+}^{k}=Q^{+}\left(D_{x}-\Lambda^{-}\right) u^{k}, \quad w_{-}^{k}=Q^{-}\left(D_{x}-\widetilde{\Lambda}^{+}\right) u^{k},
$$


solutions de

$$
\begin{aligned}
& \left(D_{x}-\Lambda^{+}\right) w_{+}^{k}=\left[D_{x}-\Lambda^{+}, Q^{+}\right]\left(D_{x}-\Lambda^{-}\right) u^{k}+Q^{+} v^{k}, \\
& \left(D_{x}-\widetilde{\Lambda}^{-}\right) w_{-}^{k}=\left[D_{x}-\widetilde{\Lambda}^{-}, Q^{-}\right]\left(D_{x}-\widetilde{\Lambda}^{+}\right) u^{k}+Q^{-} v^{k} .
\end{aligned}
$$

Les termes de droite dans (A.33) et (A.34) sont bornés dans $L^{2}$. Les inégalités d'énergie usuelles (voir $[8, \S 23.1]$ ) pour l'opérateur $\left(D_{x}-\Lambda^{+}\right)$montrent (puisque $w_{+}^{k} \equiv 0$ pour $x \gg 0$ ) que

$$
w_{+}^{k} \in C\left([0,1] ; L^{2}(Y)\right) .
$$

On a le même résultat pour $w_{-}^{k}$, ce qui implique que $\left.\left(Q\left(D_{x}-\Lambda^{+}\right) u^{k}\right)\right|_{x=0}$ et $\left(Q\left(D_{x}-\right.\right.$ $\left.\left.\widetilde{\Lambda}_{-}\right) u^{k}\right)\left.\right|_{x=0}$ sont bornés dans $L^{2}$, dont on déduit que $\left.D_{x} u^{k}\right|_{x=0}$ et $\left.u^{k}\right|_{x=0}$ sont bornés dans $L_{\varrho_{0}}^{2}$ et $H_{\varrho_{0}}^{1}$ respectivement.

On revient à l'analyse de (A.33).

Puisque le symbole principal de $\left[D_{x}-\Lambda^{+}, Q^{+}\right]$est égal d'après (A.31) à

$$
\frac{1}{i}\left(\partial_{x}\left(q_{0}\right)-\left\{\sqrt{r}, q_{0}\right\}\right)+\frac{1}{i}\left(\partial_{x}(\varphi)-\{\sqrt{r}, \varphi\} q_{0}\right)=-i \varphi^{\prime}(x) q_{0},
$$

la seule contribution qui ne converge pas fortement vers 0 dans $L^{2}$ dans le membre de droite de (A.33) est celle de l'intersection du support de $q_{0}$ (un petit voisinage de $\gamma^{+}$) avec le support de $\left.\varphi^{\prime}\right)$. Si on fait l'hypothèse additionnelle que $u^{k}$ converge fortement vers 0 microlocalement dans $\{x>0\}$ au voisinage la demi-bicaractéristique issue du point $\varrho=\left(x=0, y_{0}\right.$, $\left.\xi_{0}=\sqrt{r}\left(0, y_{0}, \eta_{0}\right), \eta_{0}\right)$ (ou dit autrement que le support de la mesure de défaut associée à $\left(u^{k}\right)$ ne rencontre pas, au voisinage de $\varrho_{0}$, pour $x>0$, cette demi-bicaractéristique), le terme de droite de (A.33) converge fortement vers 0 dans $L^{2}$ et on en déduit par les estimations d'énergie usuelles pour l'opérateur $\left(D_{x}-\Lambda^{+}\right)$

$$
\left.w_{k}^{+}\right|_{x=0}=Q_{0}\left(\left.D_{x} u^{k}\right|_{x=0}-\left.\Lambda^{-} u^{k}\right|_{x=0}\right) \rightarrow 0 \quad \text { dans } L^{2}(Y) .
$$

La relation (A.37) nous donne donc une relation pseudodifférentielle entre les deux traces de $u^{k}$. Si on fait l'hypothèse symétrique que le support de la mesure de défaut associée à $\left(u^{k}\right)$ ne rencontre pas, au voisinage de $\varrho_{0}$, pour $x>0$, la demi-bicaractéristique issue de $\left(x=0, y_{0}, \xi_{0}=-\sqrt{r}, \eta_{0}\right)$, on obtient une relation pseudodifférentielle symétrique

$$
\begin{aligned}
& Q_{0}\left(\left.D_{x} u^{k}\right|_{x=0}-\left.\widetilde{\Lambda}^{+} u^{k}\right|_{x=0}\right) \rightarrow 0 \text { dans } L^{2}(Y) \\
& \Leftrightarrow Q_{0}\left(\left.D_{x} u^{k}\right|_{x=0}+\left.\Lambda^{-} u^{k}\right|_{x=0}\right) \rightarrow 0 \text { dans } L^{2}(Y) .
\end{aligned}
$$

Si les deux relations pseudo-différentielles précédentes sont vérifiées, on en déduit facilement que les deux traces de $\left(u^{k}\right)$ convergent fortement vers 0 dans $H_{\varrho_{0}}^{1}$ et $L_{\varrho_{0}}^{2}$ respectivement.

\section{Remerciements}

Nous remercions le rapporteur pour ses commentaires et remarques.

\section{RÉFÉRENCES}

[1] Bardos C., Lebeau G., Rauch J., Sharp sufficient conditions for the observation, control and stabilization of waves from the boundary, SIAM J. Control Optim. 305 (1992) 1024-1065. 
[2] ChazARAin J., Piriou A., Introduction à la théorie des équations aux dérivées partielles linéaires, Gauthier-Villars, 1970.

[3] DenKer N., On the propagation of the polarization set for systems of real principal type, J. Functional Anal. 46 (1982) 351-373.

[4] GÉrard C., Propagation de la polarisation pour des problèmes aux limites convexes pour les bicaractéristiques, Comm. Partial Differential Equations 10 (1985) 1347-1382.

[5] GÉrard P., Microlocal defect measures, Comm. Partial Differential Equations 16 (1991) 1761-1794.

[6] GÉrard P., Leichtnam E., Ergodic properties of eigenfunctions for the Dirichlet problem, Duke Math. J. 71 (1993) 559-607.

[7] HöRmAnder L., The Analysis of Linear Partial Differential Operators I, Grundlehren der mathematischen Wissenschaften, Vol. 256, Springer Verlag, 1983.

[8] Hörmander L., The Analysis of Linear Partial Differential Operators III, Grundlehren der mathematischen Wissenschaften, Vol. 274, Springer Verlag, 1985.

[9] Koch H., Tataru D., On the spectrum of hyperbolic semigroups, Comm. Partial Differential Equations 20 (5-6) (1995) 901-937.

[10] Lebeau G., Équation des ondes amorties, in : Boutet de Monvel A., Marchenko V. (Eds.), Algebraic and Geometric Methods in Mathematical Physics, Kluwer Academic, Dordrecht, 1996, pp. 73-109.

[11] Lebeau G., Zuazua E., Decay rates for the three-dimensional linear system of thermoelasticity, Prépublications du Centre de Mathématiques de l'École Polytechnique, 98-3, 1998.

[12] Miller L., Propagation d'ondes semi-classiques à travers une interface et mesures 2-microlocales, $\mathrm{PhD}$ thesis, École Polytechnique, 1996.

[13] Melrose R.B., Sjöstrand J., Singularities of boundary value problems I, Comm. Pure Appl. Math. 31 (1978) 593-617.

[14] Melrose R.B., Sjöstrand J., Singularities of boundary value problems I \& II, Comm. Pure Appl. Math. 31 (1978) 593-617, 35 (1982) 129-168.

[15] TARTAR L., H-measures, a new approach for studying homogenization, oscillations and concentration effects in partial differential equations, Proc. Royal Soc. Edinburgh 115-A (1990) 193-230.

(Manuscrit reçu le 7 octobre 1999; accepté, après révision, le 12 octobre 2000.)

\author{
Nicolas BURQ \\ Université de Paris Sud, \\ UMR 8628 du CNRS, \\ Mathématiques, Bât. 425, \\ 91405 Orsay Cedex, France \\ E-mail : Nicolas.Burg@math.u-psud.fr \\ Gilles LEBEAU \\ Centre de Mathématiques, \\ UMR 7640 du CNRS, \\ École Polytechnique, \\ 91128 Palaiseau Cedex, France \\ E-mail : lebeau@math.polytechnique.fr
}

